\title{
GLOBAL IN TIME STRICHARTZ ESTIMATES FOR THE FRACTIONAL SCHRÖDINGER EQUATIONS ON ASYMPTOTICALLY EUCLIDEAN MANIFOLDS
}

\author{
VAN DUONG DINH
}

\begin{abstract}
In this paper, we prove global in time Strichartz estimates for the fractional Schrödinger operators, namely $e^{-i t \Lambda_{g}^{\sigma}}$ with $\sigma \in(0, \infty) \backslash\{1\}$ and $\Lambda_{g}:=\sqrt{-\Delta_{g}}$ where $\Delta_{g}$ is the Laplace-Beltrami operator on asymptotically Euclidean manifolds $\left(\mathbb{R}^{d}, g\right)$. Let $f_{0} \in C_{0}^{\infty}(\mathbb{R})$ be a smooth cutoff equal 1 near zero. We firstly show that the high frequency part $\left(1-f_{0}\right)(P) e^{-i t \Lambda_{g}^{\sigma}}$ satisfies global in time Strichartz estimates as on $\mathbb{R}^{d}$ of dimension $d \geq 2$ inside a compact set under non-trapping condition. On the other hand, under the moderate trapping assumption (1.12), the high frequency part also satisfies the global in time Strichartz estimates outside a compact set. We next prove that the low frequency part $f_{0}(P) e^{-i t \Lambda_{g}^{\sigma}}$ satisfies global in time Strichartz estimates as on $\mathbb{R}^{d}$ of dimension $d \geq 3$ without using any geometric assumption on $g$. As a byproduct, we prove global in time Strichartz estimates for the fractional Schrödinger and wave equations on $\left(\mathbb{R}^{d}, g\right), d \geq 3$ under non-trapping condition.
\end{abstract}

\section{INTRODUCTION}

Let $(M, g)$ be a $d$-dimensional Riemannian manifold. We consider the time dependent fractional Schrödinger equation on $(M, g)$, namely

$$
i \partial_{t} u-\Lambda_{g}^{\sigma} u=0, \quad u_{\mid t=0}=u_{0},
$$

with $\sigma \in(0, \infty) \backslash\{1\}, \Lambda_{g}=\sqrt{-\Delta_{g}}$ where $\Delta_{g}$ is the Laplace-Beltrami operator associated to the metric $g$. The fractional Schrödinger equation (1.1) arises in many physical contexts. When $\sigma \in(0,2) \backslash\{1\}$, the fractional Schrödinger equation was discovered by N. Laskin (see [27], [28]) as a result of extending the Feynman path integral, from the Brownian-like to Lévy-like quantum mechanical paths. This type of equation also appears in the water wave models (see [22], [32]). When $\sigma=2$, it corresponds to the well-known Schrödinger equation. In the case $\sigma=4$, it is the fourth-order Schrödinger equation introduced by Karpman [24] and Karpman and Shagalov [25] to take into account the role of small fourth-order dispersion terms in the propagation of intense laser beams in a bulk medium with Kerr nonlinearity.

When $M=\mathbb{R}^{d}$ and $g=\mathrm{Id}$, i.e. the flat Euclidean space, the solution to (1.1) enjoys the following global in time Strichartz estimates (see [16]),

$$
\|u\|_{L^{p}\left(\mathbb{R}, L^{q}\left(\mathbb{R}^{d}\right)\right)} \lesssim\left\|u_{0}\right\|_{\dot{H}^{\gamma p, q}\left(\mathbb{R}^{d}\right)},
$$

where $(p, q)$ satisfies the fractional admissible condition, i.e.

$$
p \in[2, \infty], \quad q \in[2, \infty), \quad(p, q, d) \neq(2, \infty, 2), \quad \frac{2}{p}+\frac{d}{q} \leq \frac{d}{2},
$$

2010 Mathematics Subject Classification. 35G20, 35G25.

Key words and phrases. Global in time Strichartz estimate; fractional Schrödinger equation; Littlewood-Paley decomposition, Isozaki-Kitada parametrix. 
with

$$
\gamma_{p, q}=\frac{d}{2}-\frac{d}{q}-\frac{\sigma}{p}
$$

Remark that one also has global in time Strichartz estimates for $q=\infty$, but one has to replace the Lebesgue norm $L^{\infty}\left(\mathbb{R}^{d}\right)$ by a corresponding Besov norm due to the Littlewood-Paley theorem. We refer the reader to [16] for more details.

When $M$ is a compact Riemannian manifold without boundary and $g$ is smooth, we also have (see [17]) Strichartz estimates but only local in time,

$$
\|u\|_{L^{p}\left([0,1], L^{q}(M)\right)} \lesssim\left\|u_{0}\right\|_{H^{\gamma}(M)} .
$$

In the case $\sigma \in(0,1)$, we have the same (local in time) Strichartz estimates as in ( $\mathbb{R}^{d}$, Id), i.e. $\gamma=\gamma_{p, q}$. In the case $\sigma \in(1, \infty)$, there is a "loss" of derivatives $(\sigma-1) / p$ comparing to the one on $\left(\mathbb{R}^{d}\right.$, Id), i.e. $\gamma=\gamma_{p, q}+(\sigma-1) / p$.

When $M$ is a non-compact Riemannian manifold, global in time Strichartz estimates for the Schrödinger equation (i.e. $\sigma=2$ ) have been studied intensively. In [8], Bouclet-Tzvetkov established global in time Strichartz estimates on asymptotically Euclidean manifold, i.e. $\mathbb{R}^{d}$ equipped with a long range perturbation metric $g$ (see (1.5)) with a low frequency cutoff under non-trapping condition. The first breakthrough on this topic was done by Tataru in [45] where the author considered long range and globally small perturbations of the Euclidean metric with $C^{2}$ and time dependent coefficients. In this setting, no trapping could occur. Later, Marzuola-Metcalfe-Tataru in [29] improved the results considering more general perturbations in a compact set, including some weak trapping. Afterward, Hassell-Zhang in [21] extended those results for general geometric framework of asymptotically conic manifolds and including very short range potentials with non-trapping condition. Recently, Bouclet-Mizutani in [5] established global in time Strichartz estimates for a more general class of asymptotically conic manifolds including all usual smooth long range perturbations of the Euclidean metric. After that, Zhang-Zheng [51] extended the result of Hassell-Zhang [21] and proved global in time Strichartz estimates for Schrödinger operators with potentials on assymptotically conic manifoldswith non-trapping condition. They also extended Bouclet-Mizutani's result [5] by considering Schrödinger operators with short range potentials on asymptotically conic manifolds with hyperbobic trapping condition. Recently, Zhang-Zheng [52] established global in time Strichartz estimates for Schrödinger operators on metric cone.

In order to prove Strichartz estimates on curved backgrounds, one uses the Littlewood-Paley decomposition to localize the solution in frequency. One then uses microlocal techniques to derive dispersive estimates and obtain Strichartz estimates for each spectrally localized components. By summing over all frequency pieces, one gets Strichartz estimates for the solution. For local in time Strichartz estimates, this usual scheme works very well. However, for global in time Strichartz estimates, one has to face a difficulty arising at low frequency. Due to the uncertainty principle, one can only use microlocal techniques for data supported outside compact sets at low frequency. Therefore, one has to use another technique for data supported inside compact sets. Note also that on $\mathbb{R}^{d}$, one can use the scaling technique to reduce the analysis at low frequency to the study at frequency one, but this technique does not work on manifolds in general.

The goal of this paper is to study global in time Strichartz estimates for the fractional Schrödinger equation on asymptotically Euclidean manifolds. In the case of Schrödinger equation, it can be seen as a completion of those in [8] of spatial dimensions greater than or equal to 3 . In order to achieve this goal, we will use the techniques of [5] combined with the analysis of [8]. Note that since we consider a larger range of admissible condition comparing to the sharp Schrödinger admissible condition (i.e. the inequality in (1.2) is replaced by the equality) of [5], we have to be more careful in order to apply the techniques of [5]. 
Before giving the main results, let us introduce some notations. Let $g(x)=\left(g_{j k}(x)\right)_{j, k=1}^{d}$ be a metric on $\mathbb{R}^{d}, d \geq 2$, and denote $G(x)=\left(g^{j k}(x)\right)_{j, k=1}^{d}:=g^{-1}(x)$. We consider the Laplace-Beltrami operator associated to $g$, i.e.

$$
\Delta_{g}=\sum_{j, k=1}^{d}|g(x)|^{-1} \partial_{x_{j}}\left(g^{j k}(x)|g(x)| \partial_{x_{k}}\right),
$$

where $|g(x)|:=\sqrt{\operatorname{det} g(x)}$. Throughout the paper, we assume that $g$ satisfies the following assumptions.

(1) There exists $C>0$ such that for all $x, \xi \in \mathbb{R}^{d}$,

$$
C^{-1}|\xi|^{2} \leq \sum_{j, k=1}^{d} g^{j k}(x) \xi_{j} \xi_{k} \leq C|\xi|^{2} .
$$

(2) There exists $\rho>0$ such that for all $\alpha \in \mathbb{N}^{d}$, there exists $C_{\alpha}>0$ such that for all $x \in \mathbb{R}^{d}$,

$$
\left|\partial^{\alpha}\left(g^{j k}(x)-\delta_{j k}\right)\right| \leq C_{\alpha}\langle x\rangle^{-\rho-|\alpha|} .
$$

The elliptic assumption (1.4) implies that $|g(x)|$ is bounded from below and above by positive constants. Thus for $1 \leq q \leq \infty$, the spaces $L^{q}\left(\mathbb{R}^{d}, d_{g} x\right)$ where $d_{g} x=|g(x)| d x$ and $L^{q}\left(\mathbb{R}^{d}\right)$ coincide and have equivalent norms. In the sequel, we will use the same notation $L^{q}\left(\mathbb{R}^{d}\right)$. It is well-known that $-\Delta_{g}$ is essentially self-adjoint on $C_{0}^{\infty}\left(\mathbb{R}^{d}\right)$ under the assumptions (1.4) and (1.5). We denote the unique self-adjoint extension on $L^{2}\left(\mathbb{R}^{d}\right)$ by $P$. Note that the principal symbol of $P$ is

$$
p(x, \xi)=\xi^{t} G(x) \xi=\sum_{j, k=1}^{d} g^{j k}(x) \xi_{j} \xi_{k} .
$$

Now let $\gamma \in \mathbb{R}$ and $q \in[1, \infty]$. The inhomogeneous Sobolev space $W_{g}^{\gamma, q}\left(\mathbb{R}^{d}\right)$ associated to $P$ is defined as a closure of the Schwartz space $\mathscr{S}\left(\mathbb{R}^{d}\right)$ under the norm

$$
\|u\|_{W_{g}^{\gamma, q}\left(\mathbb{R}^{d}\right)}:=\left\|\left\langle\Lambda_{g}\right\rangle^{\gamma} u\right\|_{L^{q}\left(\mathbb{R}^{d}\right)} .
$$

It is very useful to recall that for all $\gamma \in \mathbb{R}$ and $q \in(1, \infty)$, there exists $C>1$ such that

$$
C^{-1}\left\|\langle\Lambda\rangle^{\gamma} u\right\|_{L^{q}\left(\mathbb{R}^{d}\right)} \leq\|u\|_{W_{g}^{\gamma, q}\left(\mathbb{R}^{d}\right)} \leq C\left\|\langle\Lambda\rangle^{\gamma} u\right\|_{L^{q}\left(\mathbb{R}^{d}\right)},
$$

with $\langle\Lambda\rangle=\sqrt{1-\Delta}$ where $\Delta$ is the free Laplace operator on $\mathbb{R}^{d}$. This fact follows from the $L^{q}-$ boundedness of zero order pseudo-differential operators (see e.g [41, Theorem 3.1.6]). The estimates (1.7) allow us to use the Sobolev embedding as on $\mathbb{R}^{d}$. For the homogeneous Sobolev space associated to $P$, one should be careful since the Schwartz space is not a good candidate due to the singularity at 0 of $\lambda \mapsto|\lambda|^{\gamma}$. Recall that (see [18, Appendix], [47, chapter 5] and [1, Chapter 6$]$ ) on $\mathbb{R}^{d}$, the homogeneous Sobolev space $\dot{W}^{\gamma, q}\left(\mathbb{R}^{d}\right)$ is the closure of $\mathscr{L}\left(\mathbb{R}^{d}\right)$ under the norm

$$
\|u\|_{\dot{W}^{\gamma, q}\left(\mathbb{R}^{d}\right)}:=\left\|\Lambda^{\gamma} u\right\|_{L^{q}\left(\mathbb{R}^{d}\right)},
$$

where

$$
\mathscr{L}\left(\mathbb{R}^{d}\right):=\left\{u \in \mathscr{S}\left(\mathbb{R}^{d}\right) \mid D^{\alpha} \hat{u}(0)=0, \forall \alpha \in \mathbb{N}^{d}\right\} .
$$

Here $\hat{\imath}$ is the spatial Fourier transform. Since there is no Fourier transform on manifolds, we need to use the spectral theory instead. We denote

$$
\mathscr{L}_{g}\left(\mathbb{R}^{d}\right):=\left\{\varphi(P) u \mid u \in \mathscr{S}\left(\mathbb{R}^{d}\right), \varphi \in C_{0}^{\infty}((0, \infty))\right\} .
$$

We define the homogeneous Sobolev space $\dot{W}_{g}^{\gamma, q}\left(\mathbb{R}^{d}\right)$ associated to $P$ as the closure of $\mathscr{L}_{g}\left(\mathbb{R}^{d}\right)$ under the norm

$$
\|u\|_{\dot{W}_{g}^{\gamma, q}\left(\mathbb{R}^{d}\right)}:=\left\|\Lambda_{g}^{\gamma} u\right\|_{L^{q}\left(\mathbb{R}^{d}\right)} .
$$


When $q=2$, we use $H^{\gamma}\left(\mathbb{R}^{d}\right), \dot{H}^{\gamma}\left(\mathbb{R}^{d}\right), H_{g}^{\gamma}\left(\mathbb{R}^{d}\right)$ and $\dot{H}_{g}^{\gamma}\left(\mathbb{R}^{d}\right)$ instead of $W^{\gamma, 2}\left(\mathbb{R}^{d}\right), \dot{W}^{\gamma, 2}\left(\mathbb{R}^{d}\right), W_{g}^{\gamma, 2}\left(\mathbb{R}^{d}\right)$ and $\dot{H}_{g}^{\gamma}\left(\mathbb{R}^{d}\right)$ respectively. Thanks to the equivalence (1.7), we will only use the usual notation $H^{\gamma}\left(\mathbb{R}^{d}\right)$ in the sequel. It is important to note (see [4, Proposition 2.3] or [43, Lemma 2.4]) that for $d \geq 2$,

$$
\|u\|_{\dot{H}_{g}^{1}\left(\mathbb{R}^{d}\right)}^{2}=\left(\Lambda_{g} u, \Lambda_{g} u\right)=(u, P u) \simeq\|\nabla u\|_{L^{2}\left(\mathbb{R}^{d}\right)}^{2}=\|u\|_{\dot{H}^{1}\left(\mathbb{R}^{d}\right)}^{2} .
$$

By the Stone theorem, the solution to (1.1) is given by $u(t)=e^{-i t \Lambda_{g}^{\sigma}} u_{0}$. Let $f_{0} \in C_{0}^{\infty}(\mathbb{R})$ be such that $f_{0}=1$ on $[-1,1]$. We split

$$
u(t)=u_{\mathrm{low}}(t)+u_{\mathrm{high}}(t)
$$

where

$$
u_{\text {low }}(t):=f_{0}(P) e^{-i t \Lambda_{g}^{\sigma}} u_{0}, \quad u_{\text {high }}(t)=\left(1-f_{0}\right)(P) e^{-i t \Lambda_{g}^{\sigma}} u_{0}
$$

We see that $u_{\text {low }}(t)$ and $u_{\text {high }}(t)$ correspond to the low and high frequencies respectively. By the Littlewood-Paley decomposition which is very similar to the one given in [5, Subsection 4.2] (see Subsection 3.1), we split the high frequency term into two parts: inside and outside a compact set. Our first result concerns the global in time Strichartz estimates for the high frequency term inside a compact set.

Theorem 1.1. Consider $\mathbb{R}^{d}, d \geq 2$ equipped with a smooth metric $g$ satisfying (1.4), (1.5) and assume that the geodesic flow associated to $g$ is non-trapping. Then for all $\chi \in C_{0}^{\infty}\left(\mathbb{R}^{d}\right)$ and all $(p, q)$ fractional admissible, there exists $C>0$ such that for all $u_{0} \in \mathscr{L}_{g}\left(\mathbb{R}^{d}\right)$,

$$
\left\|\chi u_{\mathrm{high}}\right\|_{L^{p}\left(\mathbb{R}, L^{q}\left(\mathbb{R}^{d}\right)\right)} \leq C\left\|u_{0}\right\|_{\dot{H}_{g}^{\gamma p, q}\left(\mathbb{R}^{d}\right)} .
$$

The proof of Theorem 1.1 is based on local in time Strichartz estimates and global $L^{2}$ integrability estimates of the fractional Schrödinger operator. This strategy was first used in [44] for the Schrödinger equation. We will make use of dispersive estimates given in [17] to get Strichartz estimates with a high frequency spectral cutoff on a small time interval. Thanks to global $L^{2}$ integrability estimates, we can upgrade these local in time Strichartz estimates in to global in time Strichartz estimates. This strategy depends heavily on the non-trapping condition. We believe that one can improve this result to allow some weak trapped condition such as hyperbolic trapping in [11]. We hope to come back this interesting question in a future work.

Our next result is the following global in time Strichartz estimates for the high frequency term outside a compact set.

Theorem 1.2. Consider $\mathbb{R}^{d}, d \geq 2$ equipped with a smooth metric $g$ satisfying (1.4), (1.5) and assume that there exists $M>0$ large enough such that for all $\chi \in C_{0}^{\infty}\left(\mathbb{R}^{d}\right)$,

$$
\left\|\chi(P-\lambda \pm i 0)^{-1} \chi\right\|_{\mathcal{L}\left(L^{2}\left(\mathbb{R}^{d}\right)\right)} \chi \lambda^{M}, \quad \lambda \geq 1 .
$$

Then there exists $R>0$ large enough such that for all $(p, q)$ fractional admissible, there exists $C>0$ such that for all $u_{0} \in \mathscr{L}_{g}\left(\mathbb{R}^{d}\right)$,

$$
\left\|\mathbb{1}_{\{|x|>R\}} u_{\text {high }}\right\|_{L^{p}\left(\mathbb{R}, L^{q}\left(\mathbb{R}^{d}\right)\right)} \leq C\left\|u_{0}\right\|_{\dot{H}_{g}^{\gamma p q}\left(\mathbb{R}^{d}\right)} .
$$

The assumption (1.12) is known to hold in certain trapping situations (see e.g. [14], [33] or [11]) as well as in the non-trapping case (see [36] or [48]). We remark that under the trapping condition of [14], [33] or [11], we have

$$
\left\|\chi(P-\lambda \pm i 0)^{-1} \chi\right\|_{\mathcal{L}\left(L^{2}\left(\mathbb{R}^{d}\right)\right)} \lesssim \chi \lambda^{-1 / 2} \log \lambda, \quad \lambda \geq 1
$$

and under non-trapping condition, we have (see e.g. [10], [36]) that

$$
\left\|\chi(P-\lambda \pm i 0)^{-1} \chi\right\|_{\mathcal{L}\left(L^{2}\left(\mathbb{R}^{d}\right)\right)} \lesssim \chi \lambda^{-1 / 2}, \quad \lambda \geq 1
$$


The proof of Theorem 1.2 relies on the so called Isozaki-Kitada parametrix (see [8]) and resolvent estimates given in [5] using (1.12). Recall that the Isozaki-Kitada parametrix was first introduced on $\mathbb{R}^{d}$ to study the scattering theory of Schrödinger operators with long range potentials [23]. It was then modified and successfully used to show the Strichartz estimates for Schrödinger equation outside a compact set in many papers (see e.g. [7], [8], [9], [30], [31] or [5]).

The low frequency term in (1.10) enjoys the following global in time Strichartz estimates.

Theorem 1.3. Consider $\mathbb{R}^{d}, d \geq 3$ equipped with a smooth metric $g$ satisfying (1.4), (1.5). Then for all $(p, q)$ fractional admissible, there exists $C>0$ such that for all $u_{0} \in \mathscr{L}_{g}\left(\mathbb{R}^{d}\right)$,

$$
\left\|u_{\text {low }}\right\|_{L^{p}\left(\mathbb{R}, L^{q}\left(\mathbb{R}^{d}\right)\right)} \leq C\left\|u_{0}\right\|_{\dot{H}_{g}^{\gamma p, q}\left(\mathbb{R}^{d}\right)} .
$$

As mentioned earlier, since we consider a larger range of admissible condition than the one studied in [5], we can not apply directly the low frequency Littlewood-Paley decomposition given in [5]. We thus need a "refined" version of Littlewood-Paley decomposition. To do so, we will take the advantage of heat kernel estimates (see Subsection 3.1). As a result, we split the low frequency term into two parts: one supported outside a compact set and another one localized in a weak sense, i.e. by means of a spatial decay weight. The term with a spatial decay weight is treated easily by using global $L^{p}$ integrability estimates of the fractional Schrödinger operator at low frequency. Note that this type of global $L^{p}$ integrability estimate relies on the low frequency resolvent estimates of [6] which is available for spatial dimensions $d \geq 3$. We expect that global in time Strichartz estimates for the fractional Schrödinger equation at low frequency may hold in dimension $d=2$ as well. However, we do not know how to prove it at the moment. For the term outside a compact set, we make use of microlocal techniques and a low frequency version of the Isozaki-Kitada parametrix. We refer the reader to Section 5 for more details.

Combining Theorem 1.1, Theorem 1.2 and Theorem 1.3, we have the following result.

Theorem 1.4. Consider $\mathbb{R}^{d}, d \geq 3$ equipped with a smooth metric $g$ satisfying (1.4), (1.5) and assume that the geodesic flow associated to $g$ is non-trapping. Let $u$ be a weak solution to (1.1). Then for all $(p, q)$ fractional admissible, there exists $C>0$ such that for all $u_{0} \in \mathscr{L}_{g}\left(\mathbb{R}^{d}\right)$,

$$
\|u\|_{L^{p}\left(\mathbb{R}, L^{q}\left(\mathbb{R}^{d}\right)\right)} \leq C\left\|u_{0}\right\|_{\dot{H}_{g}^{\gamma_{p, q}}\left(\mathbb{R}^{d}\right)} .
$$

Using the homogeneous Strichartz estimate (1.15) and the Christ-Kiselev Lemma, we get the following inhomogeneous Strichartz estimates.

Proposition 1.5. Consider $\mathbb{R}^{d}, d \geq 3$ equipped with a smooth metric $g$ satisfying (1.4),(1.5) and assume that the geodesic flow associated to $g$ is non-trapping. Let $\sigma \in(0, \infty) \backslash\{1\}$ and $u$ be a weak solution to the Cauchy problem

$$
\left\{\begin{aligned}
i \partial_{t} u(t, x)-\Lambda_{g}^{\sigma} u(t, x) & =F(t, x), \quad(t, x) \in \mathbb{R} \times \mathbb{R}^{d}, \\
u(0, x) & =u_{0}(x), \quad x \in \mathbb{R}^{d},
\end{aligned}\right.
$$

with data $u_{0} \in \mathscr{L}_{g}$ and $F \in C\left(\mathbb{R}, \mathscr{L}_{g}\right)$. Then for all $(p, q)$ and $(a, b)$ fractional admissible, there exists $C>0$ such that

$$
\|u\|_{L^{p}\left(\mathbb{R}, L^{q}\left(\mathbb{R}^{d}\right)\right)}+\|u\|_{L^{\infty}\left(\mathbb{R}, \dot{H}_{g}^{\gamma_{p}, q}\left(\mathbb{R}^{d}\right)\right)} \leq C\left(\left\|u_{0}\right\|_{\dot{H}_{g}^{\gamma_{p, q}\left(\mathbb{R}^{d}\right)}}+\|F\|_{L^{a^{\prime}}\left(\mathbb{R}, L^{b^{\prime}}\left(\mathbb{R}^{d}\right)\right)}\right),
$$

provided that $(p, a) \neq(2,2)$ and

$$
\gamma_{p, q}=\gamma_{a^{\prime}, b^{\prime}}+\sigma
$$

Remark 1.6. 1. The homogeneous Strichartz estimates (1.15) and the Minkowski inequality imply

$$
\|u\|_{L^{p}\left(\mathbb{R}, L^{q}\left(\mathbb{R}^{d}\right)\right)} \leq C\left(\left\|u_{0}\right\|_{\dot{H}_{g}^{\gamma p, q}\left(\mathbb{R}^{d}\right)}+\|F\|_{L^{1}\left(\mathbb{R}, \dot{H}_{g}^{\gamma p, q}\left(\mathbb{R}^{d}\right)\right)}\right) .
$$


2. When $\sigma \in(0,2) \backslash\{1\}$, we always have $\gamma_{p, q}>0$ for any fractional admissible pair $(p, q)$ except $(p, q)=(\infty, 2)$. Thus, condition (1.18) implies that $(p, a) \neq(2,2)$, and (1.17) includes the endpoint case. When $\sigma \geq 2$, the estimates (1.17) do not include the endpoint estimate.

3. In the case $\sigma \in(0,2] \backslash\{1\}$, we can replace the homogeneous Sobolev norms in (1.17) and (1.19) by the inhomogeneous ones.

Proposition 1.7. Consider $\mathbb{R}^{d}, d \geq 3$ equipped with a smooth metric $g$ satisfying (1.4),(1.5) and assume that the geodesic flow associated to $g$ is non-trapping. Let $\sigma \in(0, \infty) \backslash\{1\}$ and $v$ be a weak solution to the Cauchy problem

$$
\left\{\begin{aligned}
\partial_{t}^{2} v(t, x)+\Lambda_{g}^{2 \sigma} v(t, x)= & F(t, x), \quad(t, x) \in \mathbb{R} \times \mathbb{R}^{d} \\
v(0, x)=v_{0}(x), & \partial_{t} v(0, x)=v_{1}(x), \quad x \in \mathbb{R}^{d},
\end{aligned}\right.
$$

with data $v_{0}, v_{1} \in \mathscr{L}_{g}$ and $F \in C\left(\mathbb{R}, \mathscr{L}_{g}\right)$. Then for all $(p, q)$ and $(a, b)$ fractional admissible, there exists $C>0$ such that

$$
\|v\|_{L^{p}\left(\mathbb{R}, L^{q}\left(\mathbb{R}^{d}\right)\right)}+\|[v]\|_{L^{\infty}\left(\mathbb{R}, \dot{H}_{g}^{\gamma_{p}, q}\left(\mathbb{R}^{d}\right)\right)} \leq C\left(\|[v](0)\|_{\dot{H}_{g}^{\gamma_{p, q}\left(\mathbb{R}^{d}\right)}}+\|F\|_{\left.L^{a^{\prime}\left(\mathbb{R}, L^{b^{\prime}}\left(\mathbb{R}^{d}\right)\right)}\right)}\right),
$$

where $[v](t):=\left(v(t), \partial_{t} v(t)\right)$ and

$$
\|[v]\|_{L^{\infty}\left(\mathbb{R}, \dot{H}_{g}^{\gamma_{p}, q}\left(\mathbb{R}^{d}\right)\right)}:=\|v\|_{L^{\infty}\left(\mathbb{R}, \dot{H}_{g}^{\gamma_{p}, q}\left(\mathbb{R}^{d}\right)\right)}+\left\|\partial_{t} v\right\|_{L^{\infty}\left(\mathbb{R}, \dot{H}_{g}^{\gamma_{p, q}-\sigma}\left(\mathbb{R}^{d}\right)\right)}
$$

provided that $(p, a) \neq(2,2)$ and

$$
\gamma_{p, q}=\gamma_{a^{\prime}, b^{\prime}}+2 \sigma .
$$

Remark 1.8. As in Remark 1.6, we have

$$
\|v\|_{L^{p}\left(\mathbb{R}, L^{q}\left(\mathbb{R}^{d}\right)\right)} \leq C\left(\|[v](0)\|_{\dot{H}_{g}^{\gamma_{p, q}\left(\mathbb{R}^{d}\right)}}+\|F\|_{L^{1}\left(\mathbb{R}, \dot{H}_{g}^{\gamma_{p}, q-\sigma}\left(\mathbb{R}^{d}\right)\right)}\right) .
$$

We finally emphasize that this paper is only devoted to study global in time Strichartz estimates. We refer the reader to [16] for applications of Strichartz estimates to the local well-posedness of the nonlinear fractional Schrödinger and wave equations.

The paper is organized as follows. In Section 2, we recall some properties of the semi-classical and rescaled pseudo-differential operators, and prove some propagation estimates related to our problem. We then prove a "refined" version of the Littlewood-Paley decomposition at low frequency and give a reduction of global in time Strichartz estimates in Section 3. In Section 4, we prove global in time Strichartz estimates inside compact sets at high frequency. Section 5 is devoted to the construction of the Isozaki-Kitada parametrix for the fractional Schrödinger operator both at high and low frequencies and to the proofs of Strichartz estimates outside compact sets. Finally, we give in Section 6 the proofs for the inhomogeneous Strichartz estimates due to the Christ-Kiselev Lemma.

Notation. In this paper the constant may change from line to line and will be denoted by the same $C$. The constants with a subscript $C_{1}, C_{2}, \ldots$ will be used when we need to compare them to one another. The notation $A \lesssim_{D} B$ means that there exists a universal constant $C>0$ depending on $D$ such that $A \leq C B$. For Banach spaces $X$ and $Y$, the notation $\|\cdot\|_{\mathcal{L}(X, Y)}$ denotes the operator norm from $X$ to $Y$ and $\|\cdot\|_{\mathcal{L}(X)}:=\|\cdot\|_{\mathcal{L}(X, X)}$. The one $T=O_{\mathcal{L}(X, Y)}(A)$ means that there exists $C>0$ such that $\|T\|_{\mathcal{L}(X, Y)} \leq C A$. In order to simplify the presentation, we shall drop the notation $\mathbb{R}^{d}$ and only write $L^{q}, \mathscr{S}, \mathscr{L}_{g}, W^{\gamma, q}, \dot{W}_{g}^{\gamma, q}, H^{\gamma}, \dot{H}_{g}^{\gamma}$.

\section{Functional Calculus and propagation estimates}

In this section, we recall some well-known results on pseudo-differential operators and prove some propagation estimates related to our problem. 
2.1. Pseudo-differential operators. Let $\mu, m \in \mathbb{R}$. We consider the symbol class $S(\mu, m)$ the space of smooth functions $a$ on $\mathbb{R}^{2 d}$ satisfying

$$
\left|\partial_{x}^{\alpha} \partial_{\xi}^{\beta} a(x, \xi)\right| \leq C_{\alpha \beta}\langle x\rangle^{\mu-|\alpha|}\langle\xi\rangle^{m-|\beta|} .
$$

In practice, we mainly use $S(\mu,-\infty):=\cap_{m \in \mathbb{R}} S(\mu, m)$.

For $a \in S(\mu, m)$ and $h \in(0,1]$, we consider the semi-classical pseudo-differential operator $O p^{h}(a)$ which is defined by

$$
O p^{h}(a) u(x)=(2 \pi h)^{-d} \iint_{\mathbb{R}^{2 d}} e^{i h^{-1}(x-y) \xi} a(x, \xi) u(y) d y d \xi .
$$

By the long range assumption (1.5), we see that $h^{2} P=O p^{h}(p)+h O p^{h}\left(p_{1}\right)$ with $p \in S(0,2)$ given in (1.6) and $p_{1} \in S(-\rho-1,1) \subset S(-1,1)$. We recall that for $a \in S\left(\mu_{1}, m_{1}\right)$ and $b \in S\left(\mu_{2}, m_{2}\right)$, the composition $O p^{h}(a) O p^{h}(b)$ is given by

$$
O p^{h}(a) O p^{h}(b)=\sum_{j=0}^{N-1} h^{j} O p^{h}\left((a \# b)_{j}\right)+h^{N} O p^{h}\left(r_{N}^{\#}(h)\right),
$$

where $(a \# b)_{j}=\sum_{|\alpha|=j} \frac{1}{\alpha !} \partial_{\xi}^{\alpha} a D_{x}^{\alpha} b \in S\left(\mu_{1}+\mu_{2}-j, m_{1}+m_{2}-j\right)$ and $\left(r_{N}^{\#}(h)\right)_{h \in(0,1]}$ is a bounded family in $S\left(\mu_{1}+\mu_{2}-N, m_{1}+m_{2}-N\right)$. The adjoint with respect to the Lebesgue measure $O p^{h}(a)^{\star}$ is given by

$$
O p^{h}(a)^{\star}=\sum_{j=0}^{N-1} h^{j} O p^{h}\left(a_{j}^{\star}\right)+h^{N} O p^{h}\left(r_{N}^{\star}(h)\right),
$$

where $a_{j}^{\star}=\sum_{|\alpha|=j} \frac{1}{\alpha !} \partial_{\xi}^{\alpha} D_{x}^{\alpha} \bar{a} \in S\left(\mu_{1}-j, m_{1}-j\right)$ and $\left(r_{N}^{\star}(h)\right)_{h \in(0,1]}$ is a bounded family in $S\left(\mu_{1}-\right.$ $\left.N, m_{1}-N\right)$.

We next recall the definition of rescaled pseudo-differential operator which is essentially given in [5]. This type of operator is very useful for the analysis at low frequency. Let $a \in S(\mu, m)$ and $\epsilon \in(0,1]$. The rescaled pseudo-differential operator $O p_{\epsilon}(a)$ is defined by

$$
O p_{\epsilon}(a) u(x)=(2 \pi)^{-d} \iint_{\mathbb{R}^{2 d}} e^{i(x-y) \xi} a\left(\epsilon x, \epsilon^{-1} \xi\right) u(y) d y d \xi .
$$

Setting $D_{\epsilon} u(x):=\epsilon^{d / 2} u(\epsilon x)$. It is easy to see that $D_{\epsilon}$ is a unitary map on $L^{2}$ and

$$
O p_{\epsilon}(a)=D_{\epsilon} O p(a) D_{\epsilon}^{-1},
$$

where $D_{\epsilon}^{-1} u(x)=\epsilon^{-d / 2} u\left(\epsilon^{-1} x\right)$ and $O p(a):=O p^{1}(a)$, i.e. $h=1$ in (2.1). Thanks to (2.2), (2.3) and (2.4), the composition $O p_{\epsilon}(a) O p_{\epsilon}(b)$ and the adjoint with respect to the Lebesgue measure $O p_{\epsilon}(a)^{\star}$ with $a \in S\left(\mu_{1}, m_{1}\right)$ and $b \in S\left(\mu_{2}, m_{2}\right)$ are given by

$$
O p_{\epsilon}(a) O p_{\epsilon}(b)=\sum_{j=0}^{N-1} O p_{\epsilon}\left((a \# b)_{j}\right)+O p_{\epsilon}\left(r_{N}^{\#}\right), \quad O p_{\epsilon}(a)^{\star}=\sum_{j=0}^{N-1} O p_{\epsilon}\left(a_{j}^{\star}\right)+O p_{\epsilon}\left(r_{N}^{\star}\right) .
$$

2.2. Functional calculus. In this subsection, we will recall the approximations for $\phi\left(h^{2} P\right)$ and $\zeta(\epsilon x) \phi\left(\epsilon^{-2} P\right)$ in terms of semi-classical and rescaled pseudo-differential operators respectively where $\phi \in C_{0}^{\infty}(\mathbb{R})$ and $\zeta \in C^{\infty}\left(\mathbb{R}^{d}\right)$ is supported outside $B(0,1)$ and equal to 1 near infinity. Here $B(0,1)$ is the open unit ball in $\mathbb{R}^{d}$.

We firstly recall the following $\mathcal{L}\left(L^{q}, L^{r}\right)$-bound of pseudo-differential operators (see e.g. [7, Proposition 2.4]). 
Proposition 2.1. Let $m>d$ and a be a continuous function on $\mathbb{R}^{2 d}$ smooth with respect to the second variable satisfying for all $\beta \in \mathbb{N}^{d}$, there exists $C_{\beta}>0$ such that for all $x, \xi \in \mathbb{R}^{d}$,

$$
\left|\partial_{\xi}^{\beta} a(x, \xi)\right| \leq C_{\beta}\langle\xi\rangle^{-m} .
$$

Then for $1 \leq q \leq r \leq \infty$, there exists $C>0$ such that for all $h \in(0,1]$,

$$
\left\|O p^{h}(a)\right\|_{\mathcal{L}\left(L^{q}, L^{r}\right)} \leq C h^{d / r-d / q} .
$$

The following proposition gives an approximation of $\phi\left(h^{2} P\right)$ in terms of semi-classical pseudodifferential operators (see e.g. [7] or [35]).

Proposition 2.2. Consider $\mathbb{R}^{d}$ equipped with a smooth metric $g$ satisfying (1.4) and (1.5). Then for a given $\phi \in C_{0}^{\infty}(\mathbb{R})$, there exist a sequence of symbols $q_{j} \in S(-j,-\infty)$ satisfying $q_{0}=\phi \circ p$ and $\operatorname{supp}\left(q_{j}\right) \subset \operatorname{supp}(\phi \circ p)$ such that for all $N \geq 1$,

$$
\phi\left(h^{2} P\right)=\sum_{j=0}^{N-1} h^{j} O p^{h}\left(q_{j}\right)+h^{N} R_{N}(h),
$$

and for $m \geq 0$ and $1 \leq q \leq r \leq \infty$, there exists $C>0$ such that for all $h \in(0,1]$,

$$
\begin{aligned}
\left\|R_{N}(h)\langle x\rangle^{N}\right\|_{\mathcal{L}\left(L^{q}, L^{r}\right)} & \leq C h^{d / r-d / q}, \\
\left\|R_{N}(h)\langle x\rangle^{N}\right\|_{\mathcal{L}\left(H^{-m}, H^{m}\right)} & \leq C h^{-2 m} .
\end{aligned}
$$

Combining Proposition 2.1 and Proposition 2.2, one has the following result (see e.g. [7, Proposition $2.9])$.

Proposition 2.3. Consider $\mathbb{R}^{d}$ equipped with a smooth metric $g$ satisfying (1.4) and (1.5). Let $\phi \in C_{0}^{\infty}(\mathbb{R})$. Then for $1 \leq q \leq r \leq \infty$, there exists $C>0$ such that for all $h \in(0,1]$,

$$
\left\|\phi\left(h^{2} P\right)\right\|_{\mathcal{L}\left(L^{q}, L^{r}\right)} \leq C h^{d / r-d / q} .
$$

It is also known (see [5]) that the rescaled pseudo-differential operator is very useful to approximate the low frequency localization of $P$, i.e. operators of the form $\phi\left(\epsilon^{-2} P\right)$. By the uncertainty principle, one can only expect to get such approximation whenever $|x|$ is large, typically $|x| \gtrsim \epsilon^{-1}$.

Remark 2.4. Let $\mu \leq 0, m \in \mathbb{R}$ and $a \in S(\mu, m)$. If we set

$$
a_{\epsilon}(x, \xi):=\epsilon^{\mu} a\left(\epsilon^{-1} x, \xi\right),
$$

then for all $\alpha, \beta \in \mathbb{N}^{d}$, there exists $C_{\alpha \beta}>0$ such that for all $|x| \geq 1, \xi \in \mathbb{R}^{d}$,

$$
\left|\partial_{x}^{\alpha} \partial_{\xi}^{\beta} a_{\epsilon}(x, \xi)\right| \leq C_{\alpha \beta}\langle\xi\rangle^{m-|\beta|}, \quad \forall \epsilon \in(0,1] .
$$

We next rewrite $\epsilon^{-2} P$ as $D_{\epsilon}\left(D_{\epsilon}^{-1}\left(\epsilon^{-2} P\right) D_{\epsilon}\right) D_{\epsilon}^{-1}$. A direct computation gives

$$
D_{\epsilon}^{-1}\left(\epsilon^{-2} P\right) D_{\epsilon}=O p\left(p_{\epsilon}\right)+O p\left(p_{\epsilon, 1}\right)=: P_{\epsilon}
$$

where $p_{\epsilon}(x, \xi)=p\left(\epsilon^{-1} x, \xi\right)$ and $p_{\epsilon, 1}(x, \xi)=\epsilon^{-1} p_{1}\left(\epsilon^{-1} x, \xi\right)$. We thus obtain

$$
\epsilon^{-2} P=O p_{\epsilon}\left(p_{\epsilon}\right)+O p_{\epsilon}\left(p_{\epsilon, 1}\right) \text {. }
$$

Using the fact that $p \in S(0,2), p_{1} \in S(-1,1)$, Remark 2.4 allows us to construct the parametrix for the resolvent $\zeta(\epsilon x)\left(\epsilon^{-2} P-z\right)^{-k}$ with $\zeta \in C^{\infty}\left(\mathbb{R}^{d}\right)$ supported outside $B(0,1)$ and equal to 1 near infinity. Indeed, by writing $\zeta(\epsilon x)\left(\epsilon^{-2} P-z\right)^{-k}=D_{\epsilon}\left[\zeta(x)\left(P_{\epsilon}-z\right)^{-k}\right] D_{\epsilon}^{-1}$, we can apply the standard elliptic parametrix for $\zeta(x)\left(P_{\epsilon}-z\right)^{-k}$ and we have (see e.g. [7] or [5]) the following result. 
Proposition 2.5. Let $\zeta, \tilde{\zeta}, \tilde{\tilde{\zeta}} \in C^{\infty}\left(\mathbb{R}^{d}\right)$ be supported outside $B(0,1)$ and equal to 1 near infinity such that $\tilde{\zeta}=1$ near $\operatorname{supp}(\zeta)$ and $\tilde{\tilde{\zeta}}=1$ near $\operatorname{supp}(\tilde{\zeta})$. Then for all $k, N \geq 1$ integers and $z \in \mathbb{C} \backslash[0,+\infty)$, we have for $\epsilon \in(0,1]$,

$$
\zeta(\epsilon x)\left(\epsilon^{-2} P-z\right)^{-k}=\sum_{j=0}^{N-1} \zeta(\epsilon x) O p_{\epsilon}\left(b_{\epsilon, j}(z)\right) \tilde{\zeta}(\epsilon x)+R_{N}(z, \epsilon),
$$

where $\left(b_{\epsilon, j}(z)\right)_{\epsilon \in(0,1]}$ is a bounded family in $S(-j,-2 k-j)$ which is a linear combination of $d_{\epsilon, l}\left(p_{\epsilon}-\right.$ $z)^{-k-l}$ with $\left(d_{\epsilon, l}\right)_{\epsilon \in(0,1]}$ a bounded family in $S(-j, 2 l-j)$ and

$$
R_{N}(z, \epsilon)=\zeta(\epsilon x) O p_{\epsilon}\left(r_{N}(z, \epsilon)\right) \tilde{\tilde{\zeta}}(\epsilon x)\left(\epsilon^{-2} P-z\right)^{-k}
$$

where $r_{N}(z, \epsilon) \in S(-N,-N)$ has seminorms growing polynomially in $1 / \operatorname{dist}\left(z, \mathbb{R}^{+}\right)$uniformly in $\epsilon \in$ $(0,1]$ as long as $z$ belongs to a bounded set of $\mathbb{C} \backslash[0,+\infty)$.

A first application of Proposition 2.5 is the following result.

Proposition 2.6. Using the notations given in Proposition 2.5, let $k>d / 2$ and $2 \leq q \leq \infty$. Then there exists $C>0$ such that for all $\epsilon \in(0,1]$,

$$
\left\|\zeta(\epsilon x)\left(\epsilon^{-2} P+1\right)^{-k}\right\|_{\mathcal{L}\left(L^{2}, L^{q}\right)} \leq C \epsilon^{d / 2-d / q} .
$$

Proof. We apply Proposition 2.5 with $N>d$, we see that

$$
\begin{aligned}
\zeta(\epsilon x)\left(\epsilon^{-2} P+1\right)^{-k} & =\sum_{j=0}^{N-1} \zeta(\epsilon x) O p_{\epsilon}\left(b_{\epsilon, j}(-1)\right) \tilde{\zeta}(\epsilon x)+\zeta(\epsilon x) O p_{\epsilon}\left(r_{N}(-1, \epsilon) \tilde{\tilde{\zeta}}(\epsilon x)\left(\epsilon^{-2} P+1\right)^{-k},\right. \\
& =\sum_{j=0}^{N-1} D_{\epsilon}\left\{\zeta(x) O p\left(b_{\epsilon, j}(-1)\right) \tilde{\zeta}(x)+\zeta(x) O p\left(r_{N}(-1, \epsilon) \tilde{\tilde{\zeta}}(x)\left(P_{\epsilon}+1\right)^{-k}\right\} D_{\epsilon}^{-1},\right.
\end{aligned}
$$

where $\left(b_{\epsilon, j}(-1)\right)_{\epsilon \in(0,1]},\left(r_{N}(-1, \epsilon)\right)_{\epsilon \in(0,1]}$ are bounded in $S(-j,-2 k-j)$ and $S(-N,-N)$ respectively. The result then follows from Proposition 2.1 with $h=1$ and that

$$
\left\|D_{\epsilon}\right\|_{\mathcal{L}\left(L^{q}\right)}=\epsilon^{d / 2-d / q}, \quad\left\|D_{\epsilon}^{-1}\right\|_{\mathcal{L}\left(L^{2}\right)}=1 .
$$

We also use that $\left\|\left(P_{\epsilon}+1\right)^{-k}\right\|_{\mathcal{L}\left(L^{2}\right)} \leq 1$ for the remainder term.

Another application of Proposition 2.5 is the following approximation of $\zeta(\epsilon x) \phi\left(\epsilon^{-2} P\right)$ in terms of rescaled pseudo-differential operators.

Proposition 2.7. Consider $\mathbb{R}^{d}$ equipped with a smooth metric $g$ satisfying (1.4) and (1.5). Let $\phi \in C_{0}^{\infty}(\mathbb{R})$ and $\zeta, \tilde{\zeta}, \tilde{\tilde{\zeta}}$ be as in Proposition 2.5. Then there exists a sequence of bounded families of symbols $\left(q_{\epsilon, j}\right)_{\epsilon \in(0,1]} \in S(-j,-\infty)$ with $q_{\epsilon, 0}=\phi \circ p_{\epsilon}$ and $\operatorname{supp}\left(q_{\epsilon, j}\right) \subset \operatorname{supp}\left(\phi \circ p_{\epsilon}\right)$ such that for all $N \geq 1$,

$$
\zeta(\epsilon x) \phi\left(\epsilon^{-2} P\right)=\sum_{j=0}^{N-1} \zeta(\epsilon x) O p_{\epsilon}\left(q_{\epsilon, j}\right) \tilde{\zeta}(\epsilon x)+R_{N}(\epsilon) .
$$

Moreover, for any $m \geq 0$, there exists $C>0$ such that for all $\epsilon \in(0,1]$,

$$
\left\|\left(\epsilon^{-2} P+1\right)^{m} R_{N}(\epsilon)\langle\epsilon x\rangle^{N}\right\|_{\mathcal{L}\left(L^{2}\right)} \leq C .
$$

Proof. By using Proposition 2.5 with $k=1$ and the Helffer-Sjöstrand formula (see [15]) namely

$$
\phi\left(\epsilon^{-2} P\right)=-\frac{1}{\pi} \int_{\mathbb{C}} \bar{\partial} \widetilde{\phi}(z)\left(\epsilon^{-2} P-z\right)^{-1} d L(z),
$$


where $\widetilde{\phi}$ is an almost analytic extension of $\phi$, the Cauchy formula gives (2.7) with

$$
R_{N}(\epsilon)=\frac{1}{\pi} \int_{\mathbb{C}} \bar{\partial} \widetilde{\phi}(z) \zeta(\epsilon x) O p_{\epsilon}\left(r_{N}(z, \epsilon)\right) \tilde{\tilde{\zeta}}(\epsilon x)\left(\epsilon^{-2} P-z\right)^{-1} d L(z) .
$$

Here $\left(r_{N}(z, \epsilon)\right)_{\epsilon \in(0,1]}$ is bounded in $S(-N,-N)$ and has semi-norms growing polynomially in $|\operatorname{Im} z|^{-1}$ which is harmless since $\bar{\partial} \widetilde{\phi}(z)=O\left(|\operatorname{Im} z|^{\infty}\right)$. The left hand side of $(2.8)$ is bounded by

$$
\frac{1}{\pi} \int_{\mathbb{C}}|\bar{\partial} \widetilde{\phi}(z)|\left\|\left(\epsilon^{-2} P+1\right)^{m} \zeta(\epsilon x) O p_{\epsilon}\left(r_{N}(z, \epsilon)\right) \tilde{\tilde{\zeta}}(\epsilon x)\left(\epsilon^{-2} P-z\right)^{-1}\langle\epsilon x\rangle^{N}\right\|_{\mathcal{L}\left(L^{2}\right)} d L(z) .
$$

By choosing $\zeta_{1} \in C^{\infty}\left(\mathbb{R}^{d}\right)$ supported outside $B(0,1)$ such that $\zeta_{1}=1$ near $\operatorname{supp}(\tilde{\tilde{\zeta}})$, we can write

$$
\left(\epsilon^{-2} P-z\right)^{-1}=\left(\epsilon^{-2} P-z\right)^{-1}\left(1-\zeta_{1}\right)(\epsilon x)+\left(\epsilon^{-2} P-z\right)^{-1} \zeta_{1}(\epsilon x) .
$$

We note that $\left(1-\zeta_{1}\right)(\epsilon x)\langle\epsilon x\rangle^{N}$ is of size $O_{\mathcal{L}\left(L^{2}\right)}(1)$ due to the compact support in $\epsilon x$, and $\left(\epsilon^{-2} P+\right.$ 1) $\left(\epsilon^{-2} P-z\right)^{-1}$ is of size $O_{\mathcal{L}\left(L^{2}\right)}\left(|\operatorname{Im} z|^{-1}\right)$ by functional calculus. Moreover, using (2.5) and the same process as in the proof of Proposition 2.6, there exists $\tau(m) \in \mathbb{N}$ such that

$$
\left\|\left(\epsilon^{-2} P+1\right)^{m} \zeta(\epsilon x) O p_{\epsilon}\left(r_{N}(z, \epsilon)\right) \tilde{\tilde{\zeta}}(\epsilon x)\left(\epsilon^{-2} P+1\right)^{-1}\right\|_{\mathcal{L}\left(L^{2}\right)} \leq C|\operatorname{Im} z|^{-\tau(m)} .
$$

This shows that

$$
\left\|\left(\epsilon^{-2} P+1\right)^{m} R_{N}(\epsilon)\left(1-\zeta_{1}\right)(\epsilon x)\langle\epsilon x\rangle^{N}\right\|_{\mathcal{L}\left(L^{2}\right)} \leq C .
$$

For the term $\left(\epsilon^{-2} P+1\right)^{m} R_{N}(\epsilon) \zeta_{1}(\epsilon x)\langle\epsilon x\rangle^{N}$, using Proposition 2.5 (by taking the adjoint), we see that

$$
\left(\epsilon^{-2} P-z\right)^{-1} \zeta_{1}(\epsilon x)=\sum_{j=0}^{N^{\prime}-1} \tilde{\zeta}_{1}(\epsilon x) O p_{\epsilon}\left(\tilde{b}_{\epsilon, j}(z)\right) \zeta_{1}(\epsilon x)+\tilde{R}_{N^{\prime}}(z, \epsilon),
$$

where $\left(\tilde{b}_{\epsilon, j}(z)\right)_{\epsilon \in(0,1]}$ is a bounded family in $S(-j,-2-j)$ and

$$
\tilde{R}_{N^{\prime}}(z, \epsilon)=\left(\epsilon^{-2} P-z\right)^{-1} \tilde{\tilde{\zeta}}_{1}(\epsilon x) O p_{\epsilon}\left(\tilde{r}_{N^{\prime}}(z, \epsilon)\right) \zeta_{1}(\epsilon x),
$$

where $\tilde{r}_{N^{\prime}}(z, \epsilon) \in S\left(-N^{\prime},-N^{\prime}\right)$ has seminorms growing polynomially in $|\operatorname{Im} z|^{-1}$ uniformly in $\epsilon \in(0,1]$. By the same argument as above, we obtain

$$
\left\|\left(\epsilon^{-2} P+1\right)^{m} R_{N}(\epsilon) \zeta_{1}(\epsilon x)\langle\epsilon x\rangle^{N}\right\|_{\mathcal{L}\left(L^{2}\right)} \leq C .
$$

Combining (2.10) and (2.11), we prove (2.8).

As a consequence of Proposition 2.7, we have the following result.

Corollary 2.8. Let $\phi \in C_{0}^{\infty}(\mathbb{R})$. Then for $2 \leq q \leq r \leq \infty$, there exists $C>0$ such that for all $\epsilon \in(0,1]$,

$$
\left\|\zeta(\epsilon x) \phi\left(\epsilon^{-2} P\right)\right\|_{\mathcal{L}\left(L^{q}, L^{r}\right)} \leq C \epsilon^{d / q-d / r} .
$$

Proof. By (2.7) and (2.8) (see also (2.9)), we can write for any $N \geq 1$ and any $m \geq 0$,

$$
\zeta(\epsilon x) \phi\left(\epsilon^{-2} P\right)=\sum_{j=0}^{N-1} \zeta(\epsilon x) O p_{\epsilon}\left(q_{\epsilon, j}\right) \tilde{\zeta}(\epsilon x)+R_{N}(\epsilon),
$$

where

$$
R_{N}(\epsilon)=\tilde{\zeta}(\epsilon x)\left(\epsilon^{-2} P+1\right)^{-m} B_{\epsilon}\langle\epsilon x\rangle^{-N}
$$

with $B_{\epsilon}=O_{\mathcal{L}\left(L^{2}\right)}(1)$ uniformly in $\epsilon \in(0,1]$. The main terms can be estimated by using Proposition 2.1 (see also the proof of Proposition 2.6). It remains to treat the remainder term. We firstly note that 
$\langle\epsilon x\rangle^{-N}=O_{\mathcal{L}\left(L^{q}, L^{2}\right)}\left(\epsilon^{d / q-d / 2}\right)$ provided $N>\frac{d(q-2)}{2 q}$. Using this bound together with $B_{\epsilon}=O_{\mathcal{L}\left(L^{2}\right)}(1)$ and (2.6), we see that

$$
\begin{aligned}
\left\|R_{N}(\epsilon)\right\|_{\mathcal{L}\left(L^{q}, L^{r}\right)} & \lesssim\left\|\tilde{\zeta}(\epsilon x)\left(\epsilon^{-2} P+1\right)^{-m}\right\|_{\mathcal{L}\left(L^{2}, L^{r}\right)}\left\|B_{\epsilon}\right\|_{\mathcal{L}\left(L^{2}\right)}\left\|\langle\epsilon x\rangle^{-N}\right\|_{\mathcal{L}\left(L^{q}, L^{2}\right)} \\
& \lesssim \epsilon^{d / 2-d / r} \epsilon^{d / q-d / 2} \lesssim \epsilon^{d / q-d / r}
\end{aligned}
$$

This proves (2.12).

Another consequence of Proposition 2.7 is the following estimate.

Corollary 2.9. Let $\phi \in C_{0}^{\infty}(\mathbb{R})$. For $m \geq 0$, there exists $C>0$ such that for all $\epsilon \in(0,1]$,

$$
\left\|\langle\epsilon x\rangle^{-m} \phi\left(\epsilon^{-2} P\right)\langle\epsilon x\rangle^{m}\right\|_{\mathcal{L}\left(L^{2}\right)} \leq C .
$$

Proof. By choosing $\zeta \in C^{\infty}\left(\mathbb{R}^{d}\right)$ supported outside $B(0,1)$ and equal to 1 near infinity, we can write $\langle\epsilon x\rangle^{-m} \phi\left(\epsilon^{-2} P\right)\langle\epsilon x\rangle^{m}$ as

$$
\langle\epsilon x\rangle^{-m} \phi\left(\epsilon^{-2} P\right) \zeta(\epsilon x)\langle\epsilon x\rangle^{m}+\langle\epsilon x\rangle^{-m} \phi\left(\epsilon^{-2} P\right)(1-\zeta)(\epsilon x)\langle\epsilon x\rangle^{m} .
$$

The $\mathcal{L}\left(L^{2}\right)$-boundedness of the first term follows from the parametrix of $\phi\left(\epsilon^{-2} P\right) \zeta(\epsilon x)$ which is obtained by taking the adjoint of (2.7). The second term follows from the fact that $(1-\zeta)(\epsilon x)\langle\epsilon x\rangle^{m}$ is bounded in $\mathcal{L}\left(L^{2}\right)$ since $1-\zeta$ vanishes outside a compact set.

2.3. Propagation estimates. In this subsection, we recall some results on resolvent estimates and prove some propagation estimates both at high and low frequencies. Let us start with the following result.

Proposition 2.10. $\quad$ 1. Consider $\mathbb{R}^{d}, d \geq 2$ equipped with a smooth metric $g$ satisfying $(1.4),(1.5)$ and suppose that the assumption (1.12) holds. Then for $k \geq 0$, there exist $C>0$ and nondecreasing $N_{k} \in \mathbb{N}$ such that for all $h \in(0,1]$ and all $\lambda$ belongs to a relatively compact interval of $(0,+\infty)$,

$$
\left\|\langle x\rangle^{-1-k}\left(h^{2} P-\lambda \mp i 0\right)^{-1-k}\langle x\rangle^{-1-k}\right\|_{\mathcal{L}\left(L^{2}\right)} \leq C h^{-N_{k}} .
$$

2. Consider $\mathbb{R}^{d}, d \geq 3$ equipped with a smooth metric $g$ satisfying (1.4),(1.5). Then for $k \geq 0$, there exists $C>0$ such that for all $\epsilon \in(0,1]$ and all $\lambda$ belongs to a relatively compact interval of $(0,+\infty)$,

$$
\left\|\langle\epsilon x\rangle^{-1-k}\left(\epsilon^{-2} P-\lambda \mp i 0\right)^{-1-k}\langle\epsilon x\rangle^{-1-k}\right\|_{\mathcal{L}\left(L^{2}\right)} \leq C .
$$

The high frequency resolvent estimates (2.14) are given in [5, Proposition 7.5] and the low frequency resolvent estimates (2.15) are given in [6, Theorem 1.2]. Note that under the non-trapping condition, the estimates (2.14) hold with $N_{k}=k+1$ (see e.g. [37, Theorem 2.8]). We next use the resolvent estimates given in Proposition 2.10 to have the following resolvent estimates for the fractional Schrödinger operator.

Proposition 2.11. Let $\sigma \in(0, \infty)$.

1. Consider $\mathbb{R}^{d}, d \geq 2$ equipped with a smooth metric $g$ satisfying (1.4),(1.5) and suppose that the assumption (1.12) holds. Then for $k \geq 0$, there exist $C>0$ and non-decreasing $N_{k} \in \mathbb{N}$ such that for all $h \in(0,1]$ and all $\mu \in I \Subset(0,+\infty)$,

$$
\left\|\langle x\rangle^{-1-k}\left(\left(h \Lambda_{g}\right)^{\sigma}-\mu \mp i 0\right)^{-1-k}\langle x\rangle^{-1-k}\right\|_{\mathcal{L}\left(L^{2}\right)} \leq C h^{-N_{k}} .
$$

2. Consider $\mathbb{R}^{d}, d \geq 3$ equipped with a smooth metric $g$ satisfying (1.4),(1.5). Then for $k \geq 0$, there exists $C>0$ such that for all $\epsilon \in(0,1]$ and all $\mu \in I \Subset(0,+\infty)$,

$$
\left\|\langle\epsilon x\rangle^{-1-k}\left(\left(\epsilon^{-1} \Lambda_{g}\right)^{\sigma}-\mu \mp i 0\right)^{-1-k}\langle\epsilon x\rangle^{-1-k}\right\|_{\mathcal{L}\left(L^{2}\right)} \leq C .
$$


Proof. We only give the proof for (2.16), the one for (2.17) is similar using (2.13). We firstly note that the estimates (2.16) are equivalent to

$$
\left\|\langle x\rangle^{-1-k}\left(\left(h \Lambda_{g}\right)^{\sigma}-\mu \mp i 0\right)^{-1-k} \phi\left(h^{2} P\right)\langle x\rangle^{-1-k}\right\|_{\mathcal{L}\left(L^{2}\right)} \leq C h^{-N_{k}},
$$

where $\phi \in C_{0}^{\infty}((0,+\infty))$ satisfying $\phi=1$ near $I$. Note that here $\Lambda_{g}=\sqrt{P}$. Next, we write $\mu=\lambda^{\sigma / 2}$ with $\lambda$ lied in a relatively compact interval of $(0,+\infty)$. By functional calculus, we write

$$
\left(h \Lambda_{g}\right)^{\sigma}-\mu \mp i 0=\left(h^{2} P-\lambda \mp i 0\right) Q\left(h^{2} P, \mu\right),
$$

where $Q(\cdot, \mu)$ is smooth and non vanishing on the support of $\phi$. This implies for all $k \geq 0$,

$$
\left(\left(h \Lambda_{g}\right)^{\sigma}-\mu \mp i 0\right)^{-1-k} \phi\left(h^{2} P\right)=\left(h^{2} P-\lambda \mp i 0\right)^{-1-k} \tilde{Q}\left(h^{2} P, \mu\right),
$$

where $\tilde{Q}\left(h^{2} P, \mu\right)=\phi\left(h^{2} P\right) Q^{-1-k}\left(h^{2} P, \mu\right)$. This allows us to approximate $\tilde{Q}\left(h^{2} P, \mu\right)$ by pseudodifferential operators by means of Proposition 2.2. Thus, we have that $\langle x\rangle^{1+k} \tilde{Q}\left(h^{2} P, \mu\right)\langle x\rangle^{-1-k}$ is of size $O_{\mathcal{L}\left(L^{2}\right)}(1)$ uniformly in $\mu \in I \Subset(0,+\infty)$ and $h \in(0,1]$. Therefore, (2.16) follows from (2.14). The proof is complete.

We now give an application of resolvent estimates given in Proposition 2.11 when $k=0$ and obtain the following global $L^{2}$ integrability estimates for the fractional Schrödinger operators both at high and low frequencies.

Proposition 2.12. Let $\sigma \in(0, \infty)$ and $f \in C_{0}^{\infty}((0,+\infty))$.

1. Consider $\mathbb{R}^{d}, d \geq 2$ equipped with a smooth metric $g$ satisfying (1.4),(1.5) and suppose that the assumption (1.12) holds. Then there exists $C>0$ such that for all $u_{0} \in L^{2}$ and all $h \in(0,1]$,

$$
\left\|\langle x\rangle^{-1} f\left(h^{2} P\right) e^{-i t h^{-1}\left(h \Lambda_{g}\right)^{\sigma}} u_{0}\right\|_{L^{2}\left(\mathbb{R}, L^{2}\right)} \leq C h^{\left(1-N_{0}\right) / 2}\left\|u_{0}\right\|_{L^{2}} .
$$

2. Consider $\mathbb{R}^{d}, d \geq 3$ equipped with a smooth metric $g$ satisfying (1.4),(1.5). Then there exists $C>0$ such that for all $u_{0} \in L^{2}$ and all $\epsilon \in(0,1]$,

$$
\left\|\langle\epsilon x\rangle^{-1} f\left(\epsilon^{-2} P\right) e^{-i t \epsilon\left(\epsilon^{-1} \Lambda_{g}\right)^{\sigma}} u_{0}\right\|_{L^{2}\left(\mathbb{R}, L^{2}\right)} \leq C \epsilon^{-1 / 2}\left\|u_{0}\right\|_{L^{2}} .
$$

Remark 2.13. $\quad$ 1. By interpolating between $L^{2}(\mathbb{R})$ and $L^{\infty}(\mathbb{R})$, we get the following $L^{p}$ integrability estimates

$$
\begin{aligned}
\left\|\langle x\rangle^{-1} f\left(h^{2} P\right) e^{-i t h^{-1}\left(h \Lambda_{g}\right)^{\sigma}} u_{0}\right\|_{L^{p}\left(\mathbb{R}, L^{2}\right)} & \leq C h^{\left(1-N_{0}\right) / p}\left\|u_{0}\right\|_{L^{2}} . \\
\left\|\langle\epsilon x\rangle^{-1} f\left(\epsilon^{-2} P\right) e^{-i t \epsilon\left(\epsilon^{-1} \Lambda_{g}\right)^{\sigma}} u_{0}\right\|_{L^{p}\left(\mathbb{R}, L^{2}\right)} & \leq C \epsilon^{-1 / p}\left\|u_{0}\right\|_{L^{2}} .
\end{aligned}
$$

2. Thanks to the fact that $P$ is non-negative, these estimates are still true for $f \in C_{0}^{\infty}(\mathbb{R} \backslash\{0\})$. Moreover, we can replace $\left\|u_{0}\right\|_{L^{2}}$ in the right hand side of (2.18) and (2.20) (resp. (2.19) and (2.21)) by $\left\|f\left(h^{2} P\right) u_{0}\right\|_{L^{2}}$ (resp. $\left.\left\|f\left(\epsilon^{-2} P\right) u_{0}\right\|_{L^{2}}\right)$. Indeed, we choose $\tilde{f} \in C_{0}^{\infty}(\mathbb{R} \backslash 0)$ such that $\tilde{f}=1$ near $\operatorname{supp}(f)$ and write $f\left(h^{2} P\right)=\tilde{f}\left(h^{2} P\right) f\left(h^{2} P\right)$. We apply (2.18) and (2.20) with $\tilde{f}$ instead of $f$. Similarly for the low frequency case.

Proof of Proposition 2.12. We again only consider the high frequency case, the low frequency one is completely similar. By the limiting absorption principle (see [34, Theorem XIII.25]), we see that $\left\|\langle x\rangle^{-1} f\left(h^{2} P\right) e^{-i t\left(h \Lambda_{g}\right)^{\sigma}} u_{0}\right\|_{L^{2}\left(\mathbb{R}, L^{2}\right)}^{2}$ is bounded by

$$
2 \pi \sup _{\substack{\mu \in \mathbb{R} \\ \epsilon>0}}\left\|\langle x\rangle^{-1} f\left(h^{2} P\right)\left(\left(h \Lambda_{g}\right)^{\sigma}-\mu-i \epsilon\right)^{-1} f\left(h^{2} P\right)\langle x\rangle^{-1}\right\|_{\mathcal{L}\left(L^{2}\right)}\left\|u_{0}\right\|_{L^{2}}^{2} .
$$

By functional calculus and the holomorphy of the resolvent, it suffices to bound $\|\langle x\rangle^{-1} f\left(h^{2} P\right)\left(\left(h \Lambda_{g}\right)^{\sigma}-\right.$ $\mu-i 0)^{-1} f\left(h^{2} P\right)\langle x\rangle^{-1} \|_{\mathcal{L}\left(L^{2}\right)}$, uniformly with respect to $\mu \in \mathbb{R}$. As a function of $h \Lambda_{g}$, the operator $f\left(h^{2} P\right)\left(\left(h \Lambda_{g}\right)^{\sigma}-\mu-i 0\right)^{-1} f\left(h^{2} P\right)$ reads $f\left(\lambda^{2}\right)\left(\lambda^{\sigma}-\mu-i 0\right)^{-1} f\left(\lambda^{2}\right)$. Assume that $\operatorname{supp}(f) \subset\left[1 / c^{2}, c^{2}\right]$ 
for some $c>1$, so $\lambda \in[1 / c, c]$.

In the case $\mu \geq 2 c^{\sigma}$ or $\mu \leq 1 / 2 c^{\sigma}$, we have that $\mu-\lambda^{\sigma} \geq c^{\sigma}$ or $\lambda^{\sigma}-\mu \geq 1 / 2 c^{\sigma}$. The functional calculus gives

$$
\left\|f\left(h^{2} P\right)\left(\left(h \Lambda_{g}\right)^{\sigma}-\mu-i 0\right)^{-1} f\left(h^{2} P\right)\right\|_{\mathcal{L}\left(L^{2}\right)} \leq 2 c^{\sigma}\|f\|_{L^{\infty}(\mathbb{R})}^{2} .
$$

Thus we can assume that $\mu \in\left[1 / 2 c^{\sigma}, 2 c^{\sigma}\right]$. Using (2.16) with $k=0$, we have

$$
\left\|\langle x\rangle^{-1}\left(\left(h \Lambda_{g}\right)^{\sigma}-\mu \mp i 0\right)^{-1}\langle x\rangle^{-1}\right\|_{\mathcal{L}\left(L^{2}\right)} \leq C h^{-N_{0}} .
$$

On the other hand, $\langle x\rangle^{-1} f\left(h^{2} P\right)\langle x\rangle$ is bounded in $\mathcal{L}\left(L^{2}\right)$ by pseudo-differential calculus. This implies

$$
\left\|\langle x\rangle^{-1} f\left(h^{2} P\right) e^{-i t\left(h \Lambda_{g}\right)^{\sigma}} u_{0}\right\|_{L^{2}\left(\mathbb{R}, L^{2}\right)} \leq C h^{-N_{0} / 2}\left\|u_{0}\right\|_{L^{2}} .
$$

By scaling in time, this gives the result.

Another application of the resolvent estimates given in Proposition 2.11 is the following local energy decays for the fractional Schrödinger operators both at high and low frequencies.

Proposition 2.14. Let $\sigma \in(0, \infty)$ and $f \in C_{0}^{\infty}(\mathbb{R} \backslash\{0\})$.

1. Consider $\mathbb{R}^{d}, d \geq 2$ equipped with a smooth metric $g$ satisfying (1.4),(1.5) and suppose that the assumption (1.12) holds. Then for $k \geq 0$, there exists $C>0$ such that for all $t \in \mathbb{R}$ and all $h \in(0,1]$,

$$
\left\|\langle x\rangle^{-1-k} e^{-i t h^{-1}\left(h \Lambda_{g}\right)^{\sigma}} f\left(h^{2} P\right)\langle x\rangle^{-1-k}\right\|_{\mathcal{L}\left(L^{2}\right)} \leq C h^{-N_{k}}\left\langle t h^{-1}\right\rangle^{-k} .
$$

2. Consider $\mathbb{R}^{d}, d \geq 3$ equipped with a smooth metric $g$ satisfying (1.4),(1.5). Then for $k \geq 0$, there exists $C>0$ such that for all $t \in \mathbb{R}$ and all $\epsilon \in(0,1]$,

$$
\left\|\langle\epsilon x\rangle^{-1-k} e^{-i t \epsilon\left(\epsilon^{-1} \Lambda_{g}\right)^{\sigma}} f\left(\epsilon^{-2} P\right)\langle\epsilon x\rangle^{-1-k}\right\|_{\mathcal{L}\left(L^{2}\right)} \leq C\langle\epsilon t\rangle^{-k} .
$$

Proof. As above, we only give the proof for the high frequency case. Using the Stone formula, the operator $e^{-i t\left(h \Lambda_{g}\right)^{\sigma}} f\left(h^{2} P\right)$ reads

$$
\frac{1}{2 i \pi} \int_{\mathbb{R}} e^{-i t \mu} f\left(\mu^{2 / \sigma}\right)\left(\left(\left(h \Lambda_{g}\right)^{\sigma}-\mu-i 0\right)^{-1}-\left(\left(h \Lambda_{g}\right)^{\sigma}-\mu+i 0\right)^{-1}\right) d \mu .
$$

We use the same trick as in [8]. By multiplying to above equality with $(i t)^{k}$ and using integration by parts in the weighted spaces $\langle x\rangle^{-1-k} L^{2}$, we see that $(i t)^{k} e^{-i t\left(h \Lambda_{g}\right)^{\sigma}} f\left(h^{2} P\right)$ is a linear combination with $l+n=k$ of terms of the form

$$
\int_{\mathbb{R}} e^{-i t \mu} \partial_{\mu}^{l}\left(f\left(\mu^{2 / \sigma}\right)\right)\left(\left(\left(h \Lambda_{g}\right)^{\sigma}-\mu-i 0\right)^{-1-n}-\left(\left(h \Lambda_{g}\right)^{\sigma}-\mu+i 0\right)^{-1-n}\right) d \mu .
$$

The compact support of $f$ implies that $\mu$ is bounded from above and below. The resolvent estimates (2.16) then imply

$$
\left\|\langle x\rangle^{-1-k} e^{-i t\left(h \Lambda_{g}\right)^{\sigma}} f\left(h^{2} P\right)\langle x\rangle^{-1-k}\right\|_{\mathcal{L}\left(L^{2}\right)} \leq C h^{-N_{k}}\langle t\rangle^{-k} .
$$

Here we use that $N_{m}$ is non-decreasing with respect to $m$. By scaling in time, we have (2.22). The proof is complete.

\section{Reduction of the PROBLEM}

3.1. The Littlewood-Paley theorems. In this subsection, we recall some Littlewood-Paley type estimates which are essentially given in [5]. Let us introduce $f(\lambda)=f_{0}(\lambda)-f_{0}(2 \lambda)$, where $f_{0}$ given as in (1.10). We have $f \in C_{0}^{\infty}(\mathbb{R} \backslash\{0\})$ and

$$
\sum_{k=1}^{\infty} f\left(2^{-k} \lambda\right)=\left(1-f_{0}\right)(\lambda), \quad \sum_{k=0}^{\infty} f\left(2^{k} \lambda\right)=\mathbb{1}_{\mathbb{R} \backslash\{0\}}(\lambda) f_{0}(\lambda), \quad \lambda \in \mathbb{R} .
$$


The Spectral Theorem implies that

$$
\left(1-f_{0}\right)(P)=\sum_{k=1}^{\infty} f\left(2^{-k} P\right), \quad f_{0}(P)=\sum_{k=0}^{\infty} f\left(2^{k} P\right) .
$$

In the second sum, we use the fact that 0 is not an eigenvalue of $P$ (see e.g. [46]).

Theorem 3.1. 1. Let $N \geq 1$ and $\chi \in C_{0}^{\infty}\left(\mathbb{R}^{d}\right)$. Then for $q \in[2, \infty)$, there exists $C>0$ such that

$$
\left\|(1-\chi)\left(1-f_{0}\right)(P) v\right\|_{L^{q}} \leq C\left(\sum_{h^{2}=2^{-k}}\left\|(1-\chi) f\left(h^{2} P\right) v\right\|_{L^{q}}^{2}+h^{N}\left\|\langle x\rangle^{-N} f\left(h^{2} P\right) v\right\|_{L^{2}}^{2}\right)^{1 / 2},
$$

for all $v \in \mathscr{S}\left(\mathbb{R}^{d}\right)$, where $k \in \mathbb{N} \backslash\{0\}$. The same estimates hold for $\chi$ in place of $1-\chi$.

2. Let $\chi \in C_{0}^{\infty}\left(\mathbb{R}^{d}\right)$ be such that $\chi(x)=1$ for $|x| \leq 1$. Then for $q \in(2, \infty)$, there exists $C>0$ such that for all $v \in L^{2}$,

$$
\left\|f_{0}(P) v\right\|_{L^{q}} \leq C\left(\sum_{\epsilon^{-2}=2^{k}}\left\|(1-\chi)(\epsilon x) f\left(\epsilon^{-2} P\right) v\right\|_{L^{q}}^{2}+\left\|\epsilon^{d / 2-d / q}\langle\epsilon x\rangle^{-1} f\left(\epsilon^{-2} P\right) v\right\|_{L^{2}}^{2}\right)^{1 / 2} .
$$

Here we use in the sum that $k \in \mathbb{N}$.

Note that the Littlewood-Paley theorem at low frequency is slightly different from the one in [5, Theorem 4.1]. In [5], Bouclet-Mizutani considered the sharp Schrödinger admissible condition. This allows to interpolate between the trivial Strichartz estimate for $(\infty, 2)$ and the endpoint Strichartz estimate for the endpoint pair $\left(2,2^{\star}\right)$. The proof of the low frequency Littlewood-Paley theorem given in [5] makes use of the homogeneous Sobolev embedding

$$
\|v\|_{L^{2^{\star}}} \leq C\left\|\Lambda_{g} v\right\|_{L^{2}}, \quad 2^{\star}=\frac{2 d}{d-2} .
$$

Since we consider a larger range of admissible condition (1.2), we can not apply this interpolation technique. To overcome this difficulty, we will take the advantage of heat kernel estimates. Our estimate (3.3) is robust and can be applied for another types of dispersive equations such as the wave or Klein-Gordon equations.

Let $K(t, x, y)$ be the kernel of the heat operator $e^{-t P}, t>0$, i.e.

$$
e^{-t P} u(x)=\int_{\mathbb{R}^{d}} K(t, x, y) u(y) d y .
$$

We recall some properties (see e.g. [12], [19]) of the heat kernel on arbitrary Riemannian manifold.

Lemma 3.2. Let $(M, g)$ be an arbitrary Riemannian manifold. Then the heat kernel $K$ satisfies the following properties:

(i) $K$ is a strictly positive $C^{\infty}$ function on $(0, \infty) \times M \times M$.

(ii) $K$ is symmetric in the space components.

(iii) (Maximum principle)

$$
\int_{M} K(t, x, y) d_{g}(y) \leq 1
$$

(iv) (Semi-group property)

$$
\int_{M} K(s, x, y) K(t, y, z) d_{g}(y)=K(s+t, x, z) .
$$


In order to obtain the heat kernel estimate, we will make use of the Nash inequality (see e.g. [39, Theorem 3.2.1]), namely

$$
\|u\|_{L^{2}} \leq C\|u\|_{L^{1}}^{\frac{2}{d+2}}\|\nabla u\|_{L^{2}}^{\frac{d}{d+2}}
$$

Note that the Nash inequality on $\mathbb{R}^{d}$ is valid for any $d \geq 1$. Thanks to (1.9), we have for $d \geq 2$,

$$
\|u\|_{L^{2}} \leq C\|u\|_{L^{1}}^{\frac{2}{d+2}}\left\|\Lambda_{g} u\right\|_{L^{2}}^{\frac{d}{d+2}} .
$$

Using (3.6), we have the following upper bound for the heat kernel.

Theorem 3.3. There exists $C>0$ such that for all $x, y \in \mathbb{R}^{d}$ and all $t>0$ such that

In particular,

$$
\begin{aligned}
& K(t, x, x) \leq C t^{-d / 2} \\
& K(t, x, y) \leq C t^{-d / 2} \exp \left(-\frac{|x-y|^{2}}{C t}\right) .
\end{aligned}
$$

$$
\left\|e^{-t P}\right\|_{\mathcal{L}\left(L^{1}, L^{\infty}\right)} \leq C t^{-d / 2}, \quad t>0 .
$$

Proof. The proof is similar to the one given in [19, Theorem 6.1] where the author shows how to get (3.7) from the homogeneous Sobolev embedding (3.4). For the reader's convenience, we give a sketch of the proof. Fix $x \in \mathbb{R}^{d}$ and denote $v(t, y)=K(t, y, x)$ and

$$
J(t):=\|v(t)\|_{L^{2}}^{2} .
$$

Using the fact that $\partial_{t} v(t, y)=-P v(t, y)$, we have

$$
J^{\prime}(t)=2\left\langle v(t), \partial_{t} v(t)\right\rangle=-2\langle v(t), P v(t)\rangle=-2\left\|\Lambda_{g} v(t)\right\|_{L^{2}}^{2} .
$$

This implies that $J(t)$ is non-increasing. On the other hand, the maximum principle (see also [19]) shows that

$$
\|v(t)\|_{L^{1}}=\int_{\mathbb{R}^{d}} K(t, x, y) d y \leq 1 .
$$

This together with (3.6) yield

$$
\|v(t)\|_{L^{2}}^{2} \leq C\|v(t)\|_{L^{1}}^{\frac{4}{d+2}}\left\|\Lambda_{g} v(t)\right\|_{L^{2}}^{\frac{2 d}{d+2}} \leq C\left\|\Lambda_{g} v(t)\right\|_{L^{2}}^{\frac{2 d}{d+2}} .
$$

We thus get

$$
J^{\prime}(t) \leq-C\|v(t)\|_{L^{2}}^{\frac{2(d+2)}{d}}=-C J(t)^{\frac{d+2}{d}} .
$$

Integrating between 0 and $t$ with the fact that the non-increasing property of $J(t)$, we obtain

$$
J(t) \leq C t^{-d / 2} .
$$

The estimate (3.7) then follows by the symmetric property of $K(t, x, y)$, i.e. $J(t)=K(2 t, x, x)$. Using (3.7), the off-diagonal argument (see also [19]) implies the following upper bound for the heat kernel

$$
K(t, x, y) \leq C t^{-d / 2} \exp \left(-\frac{d^{2}(x, y)}{C t}\right), \quad \forall x, y \in \mathbb{R}^{d}, t>0,
$$

where $d(x, y)$ is the geodesic distance from $x$ to $y$. Thanks to the elliptic condition (1.4) of the metric $g$, it is easy to see that

$$
d(x, y) \sim|x-y| .
$$

This shows (3.8) and the proof is complete.

We now give some applications of the upper bound (3.8). A first application is the following homogeneous Sobolev embedding. 
Lemma 3.4. Let $q \in(2, \infty)$ and $\alpha=\frac{d}{2}-\frac{d}{q}$. Then the operator $\Lambda_{g}^{-\alpha}$ maps $L^{2}$ to $L^{q}$. In particular, there exists $C>0$ such that

$$
\|u\|_{L^{q}} \leq C\left\|\Lambda_{g}^{\alpha} u\right\|_{L^{2}} .
$$

Proof. We firstly recall the following version of Hardy-Littlewood-Sobolev theorem.

Theorem $3.5([20,40])$. Let $1<p<q<\infty, \gamma=d+\frac{d}{q}-\frac{d}{p}$ and $K_{\gamma}(x):=|x|^{-\gamma}$. Then the convolution operator $T_{\gamma}:=f * K_{\gamma}$ maps $L^{p}$ to $L^{q}$. In particular, there exists $C>0$ such that

$$
\left\|T_{\gamma} u\right\|_{L^{q}} \leq C\|u\|_{L^{p}} .
$$

Now let $\Gamma(z):=\int_{0}^{\infty} t^{z-1} e^{-t} d t, \operatorname{Re}(z)>0$ be the Gamma function. The spectral theory with the fact $\Lambda_{g}=\sqrt{P}$ gives

$$
\Lambda_{g}^{-\alpha}=P^{-\alpha / 2}=\frac{1}{\Gamma(\alpha / 2)} \int_{0}^{\infty} e^{-t P} t^{\alpha / 2-1} d t .
$$

Let $\left[\Lambda_{g}^{-\alpha}\right](x, y)$ be the kernel of $\Lambda_{g}^{-\alpha}$. By (3.8),

$$
\left|\left[\Lambda_{g}^{-\alpha}\right](x, y)\right| \leq \frac{C}{\Gamma(\alpha / 2)} \int_{0}^{\infty} t^{-d / 2} e^{-\frac{|x-y|^{2}}{C t}} t^{\alpha / 2-1} d t .
$$

A change of variable shows

$$
\left|\left[\Lambda_{g}^{-\alpha}\right](x, y)\right| \leq \frac{C}{\Gamma(\alpha / 2)}|x-y|^{-(d-\alpha)} \int_{0}^{\infty} t^{d / 2-\alpha / 2-1} e^{-t} d t=\frac{C \Gamma(d / 2-\alpha / 2)}{\Gamma(\alpha / 2)}|x-y|^{-(d-\alpha)} .
$$

The result follows by applying Theorem 3.5 with $\gamma=d-\alpha$ and $p=2$.

Another application of the heat kernel upper bound (3.8) is the following $L^{q}-L^{r}$-bound of the heat operator.

Lemma 3.6. Let $1 \leq q \leq r \leq \infty$. The heat operator $e^{-t P}, t>0$ maps $L^{q}$ to $L^{r}$. In particular, there exists $C>0$ such that for all $t>0$,

$$
\left\|e^{-t P}\right\|_{\mathcal{L}\left(L^{q}, L^{r}\right)} \leq C t^{-\frac{d}{2}\left(\frac{1}{q}-\frac{1}{r}\right)} .
$$

Proof. By the symmetric and maximal principle properties of the heat kernel, the Schur's Lemma yields

$$
\left\|e^{-t P}\right\|_{\mathcal{L}\left(L^{q}\right)} \leq C, \quad t>0 .
$$

Interpolating between (3.9) and (3.11), we have the result.

Corollary 3.7. Let $f \in C_{0}^{\infty}(\mathbb{R} \backslash\{0\})$ and $q \in[2, \infty]$. Then there exists $C>0$ such that for all $\epsilon \in(0,1]$,

$$
\left\|f\left(\epsilon^{-2} P\right)\right\|_{\mathcal{L}\left(L^{2}, L^{q}\right)} \leq C \epsilon^{d / 2-d / q} .
$$

Proof. By writing

$$
f\left(\epsilon^{-2} P\right)=e^{-\epsilon^{-2} P}\left(e^{\epsilon^{-2} P} f\left(\epsilon^{-2} P\right)\right),
$$

and using Proposition 3.6 with $t=\epsilon^{-2}$, we get

$$
\left\|f\left(\epsilon^{-2} P\right)\right\|_{\mathcal{L}\left(L^{2}, L^{q}\right)} \leq\left\|e^{-\epsilon^{-2} P}\right\|_{\mathcal{L}\left(L^{2}, L^{q}\right)}\left\|e^{\epsilon^{-2} P} f\left(\epsilon^{-2} P\right)\right\|_{\mathcal{L}\left(L^{2}\right)} \leq C \epsilon^{d / 2-d / q} .
$$

Here, using the compactly supported property of $f$ and spectral theorem, we have $e^{\epsilon^{-2} P} f\left(\epsilon^{-2} P\right)$ is of size $O_{\mathcal{L}\left(L^{2}\right)}(1)$. This gives the result. 
We now are able to prove Theorem 3.1. We only give the proof for the low frequency case. The high frequency one is essentially given in [5, Theorem 4.6].

Proof of Theorem 3.1. By the second term of (3.1), we have

$$
\left\|f_{0}(P) v\right\|_{L^{q}}=\sup _{\|w\|_{L^{\prime}}=1}\left|\left(w, f_{0}(P) v\right)\right|=\sup _{\|w\|_{L q^{\prime}}}\left|\lim _{M \rightarrow \infty} \sum_{k=0}^{M}\left(w, f\left(\epsilon^{-2} P\right) v\right)\right|,
$$

where $\epsilon^{-2}=2^{k}$ and $(\cdot, \cdot)$ is the inner product on $L^{2}$. By choosing $\tilde{f} \in C_{0}^{\infty}(\mathbb{R} \backslash\{0\})$ satisfying $\tilde{f}=1$ near $\operatorname{supp}(f)$, we use Proposition 2.7 to write $(1-\chi)(\epsilon x) \tilde{f}\left(\epsilon^{-2} P\right)=Q(\epsilon)+R(\epsilon)$, where

$$
Q(\epsilon)=(1-\chi)(\epsilon x) O p_{\epsilon}\left(\tilde{f} \circ p_{\epsilon}\right) \zeta(\epsilon x), \quad R(\epsilon)=\zeta(\epsilon x)\left(\epsilon^{-2} P+1\right)^{-m} B(\epsilon)\langle\epsilon x\rangle^{-1},
$$

with $\zeta \in C^{\infty}\left(\mathbb{R}^{d}\right)$ supported outside $B(0,1)$ and equal to 1 near $\operatorname{supp}(1-\chi)$ and $B(\epsilon)=O_{\mathcal{L}\left(L^{2}\right)}(1)$ uniformly in $\epsilon \in(0,1]$. We next write

$$
f\left(\epsilon^{-2} P\right)=Q(\epsilon)(1-\chi)(\epsilon x) f\left(\epsilon^{-2} P\right)+A(\epsilon) \epsilon^{\alpha}\langle\epsilon x\rangle^{-1} f\left(\epsilon^{-2} P\right),
$$

with $\alpha=d / 2-d / q$ and

$$
A(\epsilon)=\epsilon^{-\alpha}\left((1-\chi)(\epsilon x) \tilde{f}\left(\epsilon^{-2} P\right) \chi(\epsilon x)+R(\epsilon)(1-\chi)(\epsilon x)+\chi(\epsilon x) \tilde{f}\left(\epsilon^{-2} P\right)\right)\langle\epsilon x\rangle .
$$

We now bound

$$
\begin{aligned}
\left|\sum_{k=0}^{M}\left(w, f\left(\epsilon^{-2} P\right) v\right)\right| \lesssim\left|\sum_{k=0}^{M}\left(w, Q(\epsilon)(1-\chi)(\epsilon x) f\left(\epsilon^{-2} P\right) v\right)\right|+\left|\sum_{k=0}^{M}\left(w, A(\epsilon) \epsilon^{\alpha}\langle\epsilon x\rangle^{-1} f\left(\epsilon^{-2} P\right) v\right)\right| \\
\quad \lesssim\left|\sum_{k=0}^{M}\left(Q^{\star}(\epsilon) w,(1-\chi)(\epsilon x) f\left(\epsilon^{-2} P\right) v\right)\right|+\|w\|_{L^{q^{\prime}}}\left\|\sum_{k=0}^{M} A(\epsilon) \epsilon^{\alpha}\langle\epsilon x\rangle^{-1} f\left(\epsilon^{-2} P\right) v\right\|_{L^{q}} \\
=:(\mathrm{I})+(\mathrm{II}) .
\end{aligned}
$$

We use the Cauchy-Schwarz inequality in $k$ and the Hölder inequality in space to have

$$
(\mathrm{I}) \lesssim\left\|\widetilde{S}_{M} w\right\|_{L^{q^{\prime}}}\left\|S_{M} v\right\|_{L^{q}}
$$

where

$$
\widetilde{S}_{M} w:=\left(\sum_{k=0}^{M}\left|Q^{\star}(\epsilon) w\right|^{2}\right)^{1 / 2}, \quad S_{M} v:=\left(\sum_{k=0}^{M}\left|(1-\chi)(\epsilon x) f\left(\epsilon^{-2} P\right) v\right|^{2}\right)^{1 / 2} .
$$

We now make use of the following estimate (see [5, Proposition 4.3]).

Proposition 3.8. For $r \in(1,2]$, there exists $C>0$ such that for all $M \geq 0$ and all $w \in \mathscr{S}\left(\mathbb{R}^{d}\right)$,

$$
\left\|\widetilde{S}_{M} w\right\|_{L^{r}} \leq C\|w\|_{L^{r}}
$$

We thus get

$$
(\mathrm{I}) \lesssim\left\|S_{M} v\right\|_{L^{q}}\|w\|_{L^{q^{\prime}}} \lesssim\left(\sum_{k=0}^{M}\left\|(1-\chi)(\epsilon x) f\left(\epsilon^{-2} P\right) v\right\|_{L^{q}}^{2}\right)^{1 / 2}\|w\|_{L^{q^{\prime}}} .
$$

For the second term in (3.13), we use the homogeneous Sobolev embedding (3.10) to have

$$
\left\|\sum_{k=0}^{M} A(\epsilon) \epsilon^{\alpha}\langle\epsilon x\rangle^{-1} f\left(\epsilon^{-2} P\right) v\right\|_{L^{q}} \lesssim\left\|\sum_{k=0}^{M} \Lambda_{g}^{\alpha} A(\epsilon) \epsilon^{\alpha}\langle\epsilon x\rangle^{-1} f\left(\epsilon^{-2} P\right) v\right\|_{L^{2}} .
$$

We next write

$$
\Lambda_{g}^{\alpha} A(\epsilon)=\left(\epsilon^{-2} P\right)^{\alpha / 2}\left(\epsilon^{-2} P+1\right)^{-\alpha} D(\epsilon)
$$


with $D(\epsilon)=O_{\mathcal{L}\left(L^{2}\right)}(1)$ uniformly in $\epsilon \in(0,1]$. It is easy to have (3.15) from the first two terms in $A(\epsilon)$ by using Proposition 2.7. The less obvious contribution in (3.14) is the uniform $L^{2}$ boundedness of $\left(\epsilon^{-2} P+1\right)^{\alpha} \chi(\epsilon x) \tilde{f}\left(\epsilon^{-2} P\right)\langle\epsilon x\rangle$. By the functional calculus, it is enough to show for $N$ large enough the uniform $L^{2}$ boundedness of $\left(\epsilon^{-2} P+1\right)^{N} \chi(\epsilon x) \tilde{f}\left(\epsilon^{-2} P\right)\langle\epsilon x\rangle$. To see it, we write

$\left(\epsilon^{-2} P+1\right)^{N} \chi(\epsilon x) \tilde{f}\left(\epsilon^{-2} P\right)\langle\epsilon x\rangle=\chi(\epsilon x)\left(\epsilon^{-2} P+1\right)^{N} \tilde{f}\left(\epsilon^{-2} P\right)\langle\epsilon x\rangle+\left[\left(\epsilon^{-2} P+1\right)^{N}, \chi(\epsilon x)\right] \tilde{f}\left(\epsilon^{-2} P\right)\langle\epsilon x\rangle$,

where $[\cdot, \cdot]$ is the commutator. The $L^{2}$ boundedness of $\chi(\epsilon x)\left(\epsilon^{-2} P+1\right)^{N} \tilde{f}\left(\epsilon^{-2} P\right)\langle\epsilon x\rangle$ follows as in (2.13). On the other hand, note that the commutator $\left[\left(\epsilon^{-2} P+1\right)^{N}, \chi(\epsilon x)\right]$ can be written as a sum of rescaled pseudo-differential operators vanishing outside the support of $\zeta(\epsilon x)$ for some $\zeta \in C^{\infty}\left(\mathbb{R}^{d}\right)$ supported outside $B(0,1)$ and equal to 1 near infinity. This allows to use Proposition 2.7, and the $L^{2}$ boundedness of $\left[\left(\epsilon^{-2} P+1\right)^{N}, \chi(\epsilon x)\right] \tilde{f}\left(\epsilon^{-2} P\right)\langle\epsilon x\rangle$ follows. We next need to recall the following well-known discrete Schur estimate.

Lemma 3.9. Let $\theta>0$ and $\left(T_{l}\right)_{l}$ be a sequence of linear operators on a Hilbert space $\mathcal{H}$. If $\left\|T_{l}^{\star} T_{k}\right\|_{\mathcal{L}(\mathcal{H})} \lesssim 2^{-\theta|k-l|}$, then there exits $C>0$ such that for all sequence $\left(v_{k}\right)_{k}$ of $\mathcal{H}$,

$$
\left\|\sum T_{k} v_{k}\right\|_{\mathcal{H}} \leq C\left(\sum\left\|v_{k}\right\|_{\mathcal{H}}^{2}\right)^{1 / 2} .
$$

Now let $T_{k}=\left(\epsilon_{k}^{-2} P\right)^{\alpha / 2}\left(\epsilon_{k}^{-2} P+1\right)^{-\alpha} D\left(\epsilon_{k}\right)$ with $\epsilon_{k}^{-2}=2^{k}$. We see that

$$
T_{l}^{\star} T_{k}=2^{\frac{\alpha(l+k)}{2}} D^{\star}\left(\epsilon_{l}\right)\left(2^{l} P+1\right)^{-\alpha} P^{\alpha}\left(2^{k} P+1\right)^{-\alpha} D\left(\epsilon_{k}\right) .
$$

Note that $l+k=-|k-l|+2 k$ for $k \geq l$ and $l+k=-|k-l|+2 l$ for $l \geq k$. Thus for $k \geq l$,

$$
\left\|T_{l}^{\star} T_{k}\right\|_{\mathcal{L}\left(L^{2}\right)}=2^{-\frac{\alpha|k-l|}{2}}\left\|D^{\star}\left(\epsilon_{l}\right)\left(2^{l} P+1\right)^{-\alpha}\left(2^{k} P\right)^{\alpha}\left(2^{k} P+1\right)^{-\alpha} D\left(\epsilon_{k}\right)\right\|_{\mathcal{L}\left(L^{2}\right)} \lesssim 2^{-\frac{\alpha|k-l|}{2}} .
$$

Similarly for $l \geq k$. Therefore, we can apply Lemma 3.9 for $T_{k}=\left(\epsilon_{k}^{-2} P\right)^{\alpha / 2}\left(\epsilon_{k}^{-2} P+1\right)^{-\alpha} D\left(\epsilon_{k}\right)$ with $\epsilon_{k}^{-2}=2^{k}, \mathcal{H}=L^{2}$ and $\theta=\alpha / 2$ to get

$$
\sup _{M}\left\|\sum_{k=0}^{M} \Lambda_{g}^{\alpha} A(\epsilon) \epsilon^{\alpha}\langle\epsilon x\rangle^{-1} f\left(\epsilon^{-2} P\right) v\right\|_{L^{2}} \lesssim\left(\sum_{k \geq 0}\left\|\epsilon^{\alpha}\langle\epsilon x\rangle^{-1} f\left(\epsilon^{-2} P\right) v\right\|_{L^{2}}^{2}\right)^{1 / 2} .
$$

Collecting (3.12), (3.13), (3.14) and (3.16), we prove (3.3). The proof of Theorem 3.1 is now complete.

3.2. Reduction of the high frequency problem. Let us now consider the high frequency case. For a given $\chi \in C_{0}^{\infty}\left(\mathbb{R}^{d}\right)$, we write $u_{\text {high }}=\chi u_{\text {high }}+(1-\chi) u_{\text {high }}$. Using (3.2) and Minkowski inequality with $p, q \geq 2$, we have

$$
\begin{aligned}
\left\|(1-\chi) u_{\text {high }}\right\|_{L^{p}\left(\mathbb{R}, L^{q}\right)} \leq C\left(\sum_{h^{2}=2^{-k}}\left\|(1-\chi) f\left(h^{2} P\right) e^{-i t \Lambda_{g}^{\sigma}} u_{0}\right\|_{L^{p}\left(\mathbb{R}, L^{q}\right)}^{2}\right. & \\
& \left.+h^{N}\left\|\langle x\rangle^{-N} f\left(h^{2} P\right) e^{-i t \Lambda_{g}^{\sigma}} u_{0}\right\|_{L^{p}\left(\mathbb{R}, L^{2}\right)}^{2}\right)^{1 / 2} .
\end{aligned}
$$

The same estimate holds for $\left\|\chi u_{\text {high }}\right\|_{L^{p}\left(\mathbb{R}, L^{q}\right)}$ with $\chi$ in place of $1-\chi$. We can apply the Item 2 of Remark 2.13 with scaling in time for the second term in the right hand side of above quantity to get

$$
h^{N / 2}\left\|\langle x\rangle^{-N} f\left(h^{2} P\right) e^{-i t \Lambda_{g}^{\sigma}} u_{0}\right\|_{L^{p}\left(\mathbb{R}, L^{2}\right)} \leq C h^{N / 2+\left(\sigma-N_{0}\right) / p}\left\|f\left(h^{2} P\right) u_{0}\right\|_{L^{2}} .
$$

By taking $N$ large enough, this term is bounded by $h^{-\gamma_{p, q}}\left\|f\left(h^{2} P\right) u_{0}\right\|_{L^{2}}$. Thus we have the following reduction. 
Proposition 3.10. $\quad$ 1. Consider $\mathbb{R}^{d}, d \geq 2$ equipped with a smooth metric $g$ satisfying (1.4), (1.5) and suppose that the geodesic flow associated to $g$ is non-trapping. If for all $\chi \in C_{0}^{\infty}\left(\mathbb{R}^{d}\right)$ and all $(p, q)$ fractional admissible, there exists $C>0$ such that for all $u_{0} \in \mathscr{L}_{g}$ and all $h \in(0,1]$,

$$
\left\|\chi e^{-i t \Lambda_{g}^{\sigma}} f\left(h^{2} P\right) u_{0}\right\|_{L^{p}\left(\mathbb{R}, L^{q}\right)} \leq C h^{-\gamma_{p, q}}\left\|f\left(h^{2} P\right) u_{0}\right\|_{L^{2}}
$$

then

$$
\left\|\chi u_{\text {high }}\right\|_{L^{p}\left(\mathbb{R}, L^{q}\right)} \leq C\left\|u_{0}\right\|_{\dot{H}_{g}^{\gamma_{p}, q}},
$$

i.e. Theorem 1.1 holds true.

2. Consider $\mathbb{R}^{d}, d \geq 2$ equipped with a smooth metric $g$ satisfying (1.4),(1.5) and suppose that (1.12) is satisfied. If there exists $R>0$ large enough such that for all $(p, q)$ fractional admissible and all $\chi \in C_{0}^{\infty}\left(\mathbb{R}^{d}\right)$ satisfying $\chi=1$ for $|x|<R$, there exists $C>0$ such that for all $u_{0} \in \mathscr{L}_{g}$ and all $h \in(0,1]$,

$$
\left\|(1-\chi) e^{-i t \Lambda_{g}^{\sigma}} f\left(h^{2} P\right) u_{0}\right\|_{L^{p}\left(\mathbb{R}, L^{q}\right)} \leq C h^{-\gamma_{p, q}}\left\|f\left(h^{2} P\right) u_{0}\right\|_{L^{2}}
$$

then

$$
\left\|(1-\chi) u_{\mathrm{high}}\right\|_{L^{p}\left(\mathbb{R}, L^{q}\right)} \leq C\left\|u_{0}\right\|_{\dot{H}_{g}^{\gamma_{p}, q}},
$$

i.e. Theorem 1.2 holds true.

Moreover, combining (3.20) and (3.22), we have

$$
\left\|u_{\text {high }}\right\|_{L^{p}\left(\mathbb{R}, L^{q}\right)} \leq C\left\|u_{0}\right\|_{\dot{H}_{g}^{\gamma_{p}, q}}
$$

Proof. We only consider the case $1-\chi$, for $\chi$ it is similar. By using (3.18) and (3.21), we see that (3.17) implies

$$
\left\|(1-\chi) u_{\mathrm{high}}\right\|_{L^{p}\left(\mathbb{R}, L^{q}\right)} \leq C\left(\sum_{h^{2}=2^{-k}} h^{-2 \gamma_{p, q}}\left\|f\left(h^{2} P\right) u_{0}\right\|_{L^{2}}^{2}\right)^{1 / 2} \leq C\left\|u_{0}\right\|_{\dot{H}_{g}^{\gamma_{p, q}}} .
$$

Here we use the almost orthogonality and the support property of $f$ to obtain the last inequality. This proves (3.22).

3.3. Reduction of the low frequency problem. Let us consider the low frequency case. We only treat the case $q \in(2, \infty)$ since the Strichartz estimate for $(p, q)=(\infty, 2)$ is trivial. We apply the Littlewood-Paley estimates (3.3) and Minkowski inequality with $p \geq 2$ to have

$$
\begin{aligned}
\left\|u_{\text {low }}\right\|_{L^{p}\left(\mathbb{R}, L^{q}\right)} \leq C\left(\sum_{\epsilon^{-2}=2^{k}}\left\|(1-\chi)(\epsilon x) f\left(\epsilon^{-2} P\right) e^{-i t \Lambda_{g}^{\sigma}} u_{0}\right\|_{L^{p}\left(\mathbb{R}, L^{q}\right)}^{2}\right. & \\
& \left.+\left\|\epsilon^{d / 2-d / q}\langle\epsilon x\rangle^{-1} f\left(\epsilon^{-2} P\right) e^{-i t \Lambda_{g}^{\sigma}} u_{0}\right\|_{L^{p}\left(\mathbb{R}, L^{2}\right)}^{2}\right)^{1 / 2} .
\end{aligned}
$$

We use global $L^{p}$ integrability estimates (2.21) with rescaling in time to bound the second term in the right hand side as

$$
\left\|\epsilon^{d / 2-d / q}\langle\epsilon x\rangle^{-1} f\left(\epsilon^{-2} P\right) e^{-i t \Lambda_{g}^{\sigma}} u_{0}\right\|_{L^{p}\left(\mathbb{R}, L^{2}\right)} \leq C \epsilon^{\gamma_{p, q}}\left\|f\left(\epsilon^{-2} P\right) u_{0}\right\|_{L^{2}} .
$$

Here we recall that $\gamma_{p, q}=d / 2-d / q-\sigma / p$. This leads to the following reduction.

Proposition 3.11. Consider $\mathbb{R}^{d}, d \geq 3$ equipped with a smooth metric $g$ satisfying (1.4), (1.5). If for all $\chi \in C_{0}^{\infty}\left(\mathbb{R}^{d}\right)$ satisfying $\chi(x)=1$ for $|x| \leq 1$ and all $(p, q)$ fractional admissible, there exists $C>0$ such that for all $u_{0} \in \mathscr{L}_{g}$ and all $\epsilon \in(0,1]$,

$$
\left\|(1-\chi)(\epsilon x) f\left(\epsilon^{-2} P\right) e^{-i t \Lambda_{g}^{\sigma}} u_{0}\right\|_{L^{p}\left(\mathbb{R}, L^{q}\right)} \leq C \epsilon^{\gamma_{p, q}}\left\|f\left(\epsilon^{-2} P\right) u_{0}\right\|_{L^{2}},
$$

then

$$
\left\|u_{\text {low }}\right\|_{L^{p}\left(\mathbb{R}, L^{q}\right)} \leq C\left\|u_{0}\right\|_{\dot{H}_{g}^{\gamma_{p}, q}} .
$$


Proof. Indeed, if the estimates (3.24) hold true, then the Littlewood-Paley estimates (3.3) and (3.23) give

$$
\left\|u_{\mathrm{low}}\right\|_{L^{p}\left(\mathbb{R}, L^{q}\right)} \leq C\left(\sum_{\epsilon^{-2}=2^{k}} \epsilon^{2 \gamma_{p, q}}\left\|f\left(\epsilon^{-2} P\right) u_{0}\right\|_{L^{2}}^{2}\right)^{1 / 2}
$$

Note that

$$
\epsilon^{\gamma_{p, q}}\left\|f\left(\epsilon^{-2} P\right) u_{0}\right\|_{L^{2}} \leq \epsilon^{\gamma_{p, q}}\left\|\tilde{f}\left(\epsilon^{-2} P\right) \Lambda_{g}^{-\gamma_{p, q}}\right\|_{\mathcal{L}\left(L^{2}\right)}\left\|f\left(\epsilon^{-2} P\right) \Lambda_{g}^{\gamma_{p, q}} u_{0}\right\|_{L^{2}},
$$

where $\tilde{f} \in C_{0}^{\infty}(\mathbb{R} \backslash\{0\})$ satisfies $\tilde{f}=1$ near $\operatorname{supp}(f)$. By functional calculus, the first factor in the right hand side is bounded by

$$
\epsilon^{\gamma_{p, q}} \sup _{\lambda \in \mathbb{R}}\left|\frac{\tilde{f}\left(\epsilon^{-2} \lambda^{2}\right)}{\lambda^{\gamma_{p, q}}}\right| \leq \epsilon^{\gamma_{p, q}} \frac{\|\tilde{f}\|_{L^{\infty}(\mathbb{R})}}{(\epsilon / c)^{\gamma_{p, q}}} \leq c^{\gamma_{p, q}}\|\tilde{f}\|_{L^{\infty}(\mathbb{R})} .
$$

Here $\epsilon^{-2} \lambda^{2} \in \operatorname{supp}(\tilde{f})$ hence $|\lambda| \in[\epsilon / c, \epsilon c]$ for some constant $c>1$. Thus we have

$$
\left\|u_{\text {low }}\right\|_{L^{p}\left(\mathbb{R}, L^{q}\right)} \leq C\left(\sum_{\epsilon^{-2}=2^{k}}\left\|f\left(\epsilon^{-2} P\right) \Lambda_{g}^{\gamma_{p, q}} u_{0}\right\|_{L^{2}}^{2}\right)^{1 / 2} \leq C\left\|u_{0}\right\|_{\dot{H}_{g}^{\gamma_{p}, q}}
$$

the last inequality follows from the almost orthogonality. This completes the proof.

\section{Strichartz estimates inside Compact Sets}

In this section, we will give the proof of (3.19). Our main tools are the local in time Strichartz estimates which are proved by the WKB method (see [17]) and the $L^{2}$ integrability estimate at high frequency given in Proposition 2.12.

4.1. The WKB approximations. Let us start with the following result which is given in [17, Theorem 2.7].

Theorem 4.1. Let $\sigma \in(0, \infty) \backslash\{1\}$ and $q$ be a smooth function on $\mathbb{R}^{2 d}$ compactly support in $\xi$ away from zero and satisfying for all $\alpha, \beta \in \mathbb{N}^{d}$, there exists $C_{\alpha \beta}>0$ such that for all $x, \xi \in \mathbb{R}^{d}$,

$$
\left|\partial_{x}^{\alpha} \partial_{\xi}^{\beta} q(x, \xi)\right| \leq C_{\alpha \beta}
$$

Then there exist $t_{0}>0$ small enough, a function $S \in C^{\infty}\left(\left[-t_{0}, t_{0}\right] \times \mathbb{R}^{2 d}\right)$ and a sequence of smooth functions $a_{j}(t, x, \xi)$ compactly supported in $\xi$ away from zero uniformly in $t \in\left[-t_{0}, t_{0}\right]$ such that for all $N \geq 1$,

$$
e^{-i t h^{-1}\left(h \Lambda_{g}\right)^{\sigma}} O p^{h}(q) u_{0}=J_{N}(t) u_{0}+R_{N}(t) u_{0}
$$

where

$$
J_{N}(t) u_{0}(x)=(2 \pi h)^{-d} \iint_{\mathbb{R}^{2 d}} e^{i h^{-1}(S(t, x, \xi)-y \xi)} \sum_{j=0}^{N-1} h^{j} a_{j}(t, x, \xi) u_{0}(y) d y d \xi,
$$

$J_{N}(0)=O p^{h}(q)$ and the remainder $R_{N}(t)$ satisfies for all $t \in\left[-t_{0}, t_{0}\right]$ and all $h \in(0,1]$,

$$
\left\|R_{N}(t)\right\|_{\mathcal{L}\left(L^{2}\right)} \leq C h^{N-1} \text {. }
$$

Moreover, there exists a constant $C>0$ such that for all $t \in\left[-t_{0}, t_{0}\right]$ and all $h \in(0,1]$,

$$
\left\|J_{N}(t)\right\|_{\mathcal{L}\left(L^{1}, L^{\infty}\right)} \leq C h^{-d}\left(1+|t| h^{-1}\right)^{-d / 2} .
$$


In [17], we consider the smooth bounded metric, i.e. for all $\alpha \in \mathbb{N}^{d}$, there exists $C_{\alpha}>0$ such that for all $x \in \mathbb{R}^{d}$,

$$
\left|\partial^{\alpha} g^{j k}(x)\right| \leq C_{\alpha}, \quad j, k \in\{1, \ldots d\} .
$$

It is obvious to see that the assumption (1.5) implies (4.1). This theorem and the parametrix given in Proposition 2.2 give the following dispersive estimates for the fractional Schrödinger equations (see also [17, Remark 2.8]).

Proposition 4.2. Let $\sigma \in(0, \infty) \backslash\{1\}$ and $\varphi \in C_{0}^{\infty}(\mathbb{R} \backslash\{0\})$. Then there exists $t_{0}>0$ small enough and $C>0$ such that for all $u_{0} \in L^{1}\left(\mathbb{R}^{d}\right)$ and all $h \in(0,1]$,

$$
\left\|e^{-i t h^{-1}\left(h \Lambda_{g}\right)^{\sigma}} \varphi\left(h^{2} P\right) u_{0}\right\|_{L^{\infty}} \leq C h^{-d}\left(1+|t| h^{-1}\right)^{-d / 2}\left\|u_{0}\right\|_{L^{1}},
$$

for all $t \in\left[-t_{0}, t_{0}\right]$.

Next, we recall the following version of $T T^{\star}$-criterion of Keel and Tao (see [50], [26] or [49]).

Proposition 4.3. Let $I \subseteq \mathbb{R}$ be an interval and $(T(t))_{t \in I}$ a family of linear operators satisfying for some constant $C>0$ and $\delta, \tau, h>0$,

$$
\begin{aligned}
\|T(t)\|_{\mathcal{L}\left(L^{2}\right)} & \leq C, \\
\left\|T(t) T(s)^{\star}\right\|_{\mathcal{L}\left(L^{1}, L^{\infty}\right)} & \leq C h^{-\delta}\left(1+|t-s| h^{-1}\right)^{-\tau},
\end{aligned}
$$

for all $t, s \in I$. Then for all $(p, q)$ satisfying

$$
p \in[2, \infty], \quad q \in[1, \infty], \quad(p, q, \tau) \neq(2, \infty, 1), \quad \frac{1}{p} \leq \tau\left(\frac{1}{2}-\frac{1}{q}\right)
$$

we have

where $\kappa=\delta(1 / 2-1 / q)-1 / p$.

$$
\|T v\|_{L^{p}\left(I, L^{q}\right.} \leq C h^{-\kappa}\|v\|_{L^{2}}
$$

Proposition 4.3 together with energy estimate and dispersive estimate (4.2) give the following result.

Corollary 4.4. Let $\sigma \in(0, \infty) \backslash\{1\}, \varphi \in C_{0}^{\infty}(\mathbb{R} \backslash\{0\})$ and $t_{0}$ be as in Theorem 4.1. Denote $I=$ $\left[-t_{0}, t_{0}\right]$. Then for all $(p, q)$ fractional admissible, there exists $C>0$ such that

$$
\left\|\varphi\left(h^{2} P\right) e^{-i t h^{-1}\left(h \Lambda_{g}\right)^{\sigma}} v\right\|_{L^{p}\left(I, L^{q}\right)} \leq C h^{-\kappa_{p, q}}\|v\|_{L^{2}},
$$

where $\kappa_{p, q}=d / 2-d / q-1 / p$. Moreover,

$$
\left\|\int_{0}^{t} \varphi^{2}\left(h^{2} P\right) e^{-i(t-s) h^{-1}\left(h \Lambda_{g}\right)^{\sigma}} G(s) d s\right\|_{L^{p}\left(I, L^{q}\right)} \leq C h^{-\kappa_{p, q}}\|G\|_{L^{1}\left(I, L^{2}\right)} .
$$

Proof. The homogeneous estimates (4.5) follow directly from Proposition 4.2 and Proposition 4.3 with $T(t)=\varphi\left(h^{2} P\right) e^{-i t h^{-1}\left(h \Lambda_{g}\right)^{\sigma}}$. It remains to prove the inhomogeneous estimates (4.6). Let us set

$$
U_{h}(t):=h^{\kappa_{p, q}} \varphi\left(h^{2} P\right) e^{-i t h^{-1}\left(h \Lambda_{g}\right)^{\sigma}} .
$$

Using the homogeneous Strichartz estimates (4.5), we see that $U_{h}(t)$ is a bounded operator from $L^{2}$ to $L^{p}\left(I, L^{q}\right)$. Similarly, we have $U_{h}(s)=\varphi\left(h^{2} P\right) e^{-i s h^{-1}\left(h \Lambda_{g}\right)^{\sigma}}$ is a bounded operator from $L^{2}$ to $L^{\infty}\left(I, L^{2}\right)$. Here we use the fact that $(\infty, 2)$ is fractional admissible with $\kappa_{\infty, 2}=0$. Thus the adjoint $U_{h}(s)^{\star}$, namely

$$
U_{h}(s)^{\star}: G \in L^{1}\left(I, L^{2}\right) \mapsto \int_{I} \varphi\left(h^{2} P\right) e^{i s h^{-1}\left(h \Lambda_{g}\right)^{\sigma}} G(s) d s \in L^{2}
$$


is also a bounded operator. This implies $U_{h}(t) U_{h}(s)^{\star}$ is a bounded operator from $L^{1}\left(I, L^{2}\right)$ to $L^{p}\left(I, L^{q}\right)$. In particular, we have

$$
\left\|\int_{I} h^{\kappa_{p, q}} \varphi^{2}\left(h^{2} P\right) e^{-i(t-s) h^{-1}\left(h \Lambda_{g}\right)^{\sigma}} G(s) d s\right\|_{L^{p}\left(I, L^{q}\right)} \leq C\|G\|_{L^{1}\left(I, L^{2}\right)} .
$$

The Christ-Kiselev Lemma (see Lemma 6.1) implies that for all $(p, q)$ fractional admissible,

$$
\left\|\int_{0}^{t} \varphi^{2}\left(h^{2} P\right) e^{-i(t-s) h^{-1}\left(h \Lambda_{g}\right)^{\sigma}} G(s) d s\right\|_{L^{p}\left(I, L^{q}\right)} \leq C h^{-\kappa_{p, q}}\|G\|_{L^{1}\left(I, L^{2}\right)} .
$$

This completes the proof.

4.2. From local Strichartz estimates to global Strichartz estimates. We now show how to upgrade the local in time Strichartz estimates given in Corollary 4.4 to the global in time ones (3.19). We emphasize that the non-trapping assumption is supposed here.

Let us set $v(t)=\langle x\rangle^{-1} f\left(h^{2} P\right) e^{-i t h^{-1}\left(h \Lambda_{g}\right)^{\sigma}} u_{0}$. By choosing $f_{1} \in C_{0}^{\infty}(\mathbb{R} \backslash\{0\})$ with $f_{1}=1$ near $\operatorname{supp}(f)$, we see that the study of $\|v\|_{L^{p}\left(\mathbb{R}, L^{q}\right)}$ is reduced to the one of $\left\|f_{1}\left(h^{2} P\right) v\right\|_{L^{p}\left(\mathbb{R}, L^{q}\right)}$. Indeed, we can write

$$
v(t)=f_{1}\left(h^{2} P\right) v(t)+\left(1-f_{1}\right)\left(h^{2} P\right) v(t),
$$

where the term $\left(1-f_{1}\right)\left(h^{2} P\right) v(t)$ can be written as

$$
\left(\left(1-f_{1}\right)\left(h^{2} P\right)\langle x\rangle^{-1} \tilde{f}_{1}\left(h^{2} P\right)\langle x\rangle\right)\langle x\rangle^{-1} f\left(h^{2} P\right) e^{-i t h^{-1}\left(h \Lambda_{g}\right)^{\sigma}} u_{0},
$$

with $\tilde{f}_{1} \in C_{0}^{\infty}(\mathbb{R} \backslash\{0\})$ such that $f_{1}=1$ near $\operatorname{supp}\left(\tilde{f}_{1}\right)$ and $\tilde{f}_{1}=1$ near $\operatorname{supp}(f)$. By pseudo-differential calculus, we have

$$
\left(1-f_{1}\right)\left(h^{2} P\right)\langle x\rangle^{-1} \tilde{f}_{1}\left(h^{2} P\right)\langle x\rangle=O_{\mathcal{L}\left(L^{2}, L^{q}\right)}\left(h^{\infty}\right),
$$

for all $q \geq 2$. This implies that there exists $C>0$ such that for all $N \geq 1$,

$$
\begin{aligned}
\left\|v-f_{1}\left(h^{2} P\right) v\right\|_{L^{p}\left(\mathbb{R}, L^{q}\right)} & \leq C h^{N}\left\|\langle x\rangle^{-1} f\left(h^{2} P\right) e^{-i t h^{-1}\left(h \Lambda_{g}\right)^{\sigma}} u_{0}\right\|_{L^{p}\left(\mathbb{R}, L^{2}\right)} \\
& \leq C h^{N}\left\|f\left(h^{2} P\right) u_{0}\right\|_{L^{2}} \leq C h^{-\kappa_{p, q}}\left\|f\left(h^{2} P\right) u_{0}\right\|_{L^{2}}
\end{aligned}
$$

provided that $N$ is taken large enough. Here we use (2.20) with $N_{0}=1$ due to the non-trapping condition.

We next write

$$
v(t)=\langle x\rangle^{-1} f\left(h^{2} P\right) e^{-i t h^{-1} \psi\left(h^{2} P\right)} u_{0},
$$

where $\psi(\lambda)=\tilde{f}(\lambda) \sqrt{\lambda}^{\sigma}$ with $\tilde{f} \in C_{0}^{\infty}(\mathbb{R} \backslash\{0\})$ and $\tilde{f}=1$ near $\operatorname{supp}(f)$. Now, let $t_{0}>0$ be as in Corollary 4.4. We next choose $\theta \in C_{0}^{\infty}(\mathbb{R},[0,1])$ satisfying $\theta=1$ near 0 and $\operatorname{supp}(\theta) \subset(-1,1)$ such that $\sum_{k \in \mathbb{Z}} \theta(t-k)=1$, for all $t \in \mathbb{R}$. We then write $v(t)=\sum_{k \in \mathbb{Z}} v_{k}(t)$, where $v_{k}(t)=\theta\left(\left(t-t_{k}\right) / t_{0}\right) v(t)$ with $t_{k}=t_{0} k$. By the Duhamel formula, we have

$$
v_{k}(t)=e^{-i t h^{-1} \psi\left(h^{2} P\right)} v_{k}(0)+i h^{-1} \int_{0}^{t} e^{-i(t-s) h^{-1} \psi\left(h^{2} P\right)}\left(h D_{s}+\psi\left(h^{2} P\right)\right) v_{k}(s) d s .
$$

For $k \neq 0$, we compute the action of $h D_{s}+\psi\left(h^{2} P\right)$ on $v_{k}(s)$ and get

$$
\begin{aligned}
\left(h D_{s}+\psi\left(h^{2} P\right)\right) v_{k}(s) & =h\left(i t_{0}\right)^{-1} \theta^{\prime}\left(\left(s-t_{k}\right) / t_{0}\right) v(s) \\
& +\theta\left(\left(s-t_{k}\right) / t_{0}\right)\left[\psi\left(h^{2} P\right),\langle x\rangle^{-1}\right] f\left(h^{2} P\right) e^{-i s h^{-1} \psi\left(h^{2} P\right)} u_{0}=: v_{k}^{1}(s)+v_{k}^{2}(s) .
\end{aligned}
$$

Due to the support property of $\theta$, we have $v_{k}(0)=0$. Now, we have for $k \neq 0$,

$$
f_{1}\left(h^{2} P\right) v_{k}(t)=i h^{-1} \int_{0}^{t} e^{-i(t-s) h^{-1} \psi\left(h^{2} P\right)} f_{1}\left(h^{2} P\right)\left(v_{k}^{1}(s)+v_{k}^{2}(s)\right) d s .
$$


We remark that both $t, s$ belong to $I_{k}=\left(t_{k}-t_{0}, t_{k}+t_{0}\right)$. Up to a translation in time $t \mapsto t-t_{k}$ and the same for $s$, we can apply the inhomogeneous Strichartz estimates given in Corollary 4.4 with $\varphi^{2}=f_{1}$ and obtain

$$
\begin{aligned}
\left\|f_{1}\left(h^{2} P\right) v_{k}\right\|_{L^{p}\left(\mathbb{R}, L^{q}\right)} & =\left\|f_{1}\left(h^{2} P\right) v_{k}\right\|_{L^{p}\left(I_{k}, L^{q}\right)} \\
& \leq C h^{-\kappa_{p, q}-1}\left(\left\|v_{k}^{1}\right\|_{L^{1}\left(I_{k}, L^{2}\right)}+\left\|v_{k}^{2}\right\|_{L^{1}\left(I_{k}, L^{2}\right)}\right) .
\end{aligned}
$$

Here $\kappa_{p, q}$ is given in Corollary 4.4. We have

$$
\begin{aligned}
\left\|v_{k}^{1}\right\|_{L^{1}\left(I_{k}, L^{2}\right)} & =\left\|h\left(i t_{0}\right)^{-1} \theta^{\prime}\left(\left(s-t_{k}\right) / t_{0}\right)\langle x\rangle^{-1} f\left(h^{2} P\right) e^{-i s h^{-1}\left(h \Lambda_{g}\right)^{\sigma}} u_{0}\right\|_{L^{1}\left(I_{k}, L^{2}\right)} \\
& \leq\left\|h\left(i t_{0}\right)^{-1} \theta^{\prime}\left(\left(s-t_{k}\right) / t_{0}\right)\right\|_{L^{2}\left(I_{k}\right)}\left\|\langle x\rangle^{-1} f\left(h^{2} P\right) e^{-i s h^{-1}\left(h \Lambda_{g}\right)^{\sigma}} u_{0}\right\|_{L^{2}\left(I_{k}, L^{2}\right)} \\
& \leq C h\left\|\langle x\rangle^{-1} f\left(h^{2} P\right) e^{-i s h^{-1}\left(h \Lambda_{g}\right)^{\sigma}} u_{0}\right\|_{L^{2}\left(I_{k}, L^{2}\right)},
\end{aligned}
$$

where we use Cauchy Schwarz inequality to go from the first to the second line. Similarly

$$
\begin{aligned}
\left\|v_{k}^{2}\right\|_{L^{1}\left(I_{k}, L^{2}\right)} & \leq\left\|\left[\psi\left(h^{2} P\right),\langle x\rangle^{-1}\right] f\left(h^{2} P\right) e^{-i t h^{-1}\left(h \Lambda_{g}\right)^{\sigma}} u_{0}\right\|_{L^{2}\left(I_{k}, L^{2}\right)} \\
& \leq C h\left\|\langle x\rangle^{-1} f\left(h^{2} P\right) e^{-i t h^{-1}\left(h \Lambda_{g}\right)^{\sigma}} u_{0}\right\|_{L^{2}\left(I_{k}, L^{2}\right)},
\end{aligned}
$$

where we use the fact that $\left[\psi\left(h^{2} P\right),\langle x\rangle^{-1}\right] \tilde{f}_{1}\left(h^{2} P\right)\langle x\rangle$ is of size $O_{\mathcal{L}\left(L^{2}\right)}(h)$ by pseudo-differential calculus. This implies that for $k \neq 0$,

$$
\left\|f_{1}\left(h^{2} P\right) v_{k}\right\|_{L^{p}\left(I_{k}, L^{q}\right)} \leq C h^{-\kappa_{p, q}}\left\|\langle x\rangle^{-1} f\left(h^{2} P\right) e^{-i t h^{-1}\left(h \Lambda_{g}\right)^{\sigma}} u_{0}\right\|_{L^{2}\left(I_{k}, L^{2}\right)} .
$$

For $k=0$, we have

$$
\left\|f_{1}\left(h^{2} P\right) v_{0}\right\|_{L^{p}\left(\mathbb{R}, L^{q}\right)} \leq C\left\|f\left(h^{2} P\right) e^{-i t h^{-1}\left(h \Lambda_{g}\right)^{\sigma}} u_{0}\right\|_{L^{p}\left(I, L^{q}\right)} \leq C h^{-\kappa_{p, q}}\left\|f\left(h^{2} P\right) u_{0}\right\|_{L^{2}} .
$$

Here the first inequality follows from the facts that $\theta\left(t / t_{0}\right)$ and $f_{1}\left(h^{2} P\right)\langle x\rangle^{-1}$ are bounded in $\mathcal{L}\left(L^{p}(\mathbb{R})\right)$ and $\mathcal{L}\left(L^{q}\right)$ respectively. The second inequality follows from homogeneous Strichartz estimates (4.5). By almost orthogonality in time and the fact that $p \geq 2$, we have

$$
\begin{aligned}
\left\|f_{1}\left(h^{2} P\right) v\right\|_{L^{p}\left(\mathbb{R}, L^{q}\right)} & \leq C\left(\sum_{k \in \mathbb{Z}}\left\|f_{1}\left(h^{2} P\right) v_{k}\right\|_{L^{p}\left(\mathbb{R}, L^{q}\right)}^{2}\right)^{1 / 2} \\
& \leq C h^{-\kappa_{p, q}}\left(\sum_{k \in \mathbb{Z} \backslash 0}\left\|\langle x\rangle^{-1} f\left(h^{2} P\right) e^{-i t h^{-1}\left(h \Lambda_{g}\right)^{\sigma}} u_{0}\right\|_{L^{2}\left(I_{k}, L^{2}\right)}^{2}+\left\|f\left(h^{2} P\right) u_{0}\right\|_{L^{2}}^{2}\right)^{1 / 2} \\
& \leq C h^{-\kappa_{p, q}}\left(\left\|\langle x\rangle^{-1} f\left(h^{2} P\right) e^{-i t h^{-1}\left(h \Lambda_{g}\right)^{\sigma}} u_{0}\right\|_{L^{2}\left(\mathbb{R}, L^{2}\right)}+\left\|f\left(h^{2} P\right) u_{0}\right\|_{L^{2}}\right) \\
& \leq C h^{-\kappa_{p, q}}\left\|f\left(h^{2} P\right) u_{0}\right\|_{L^{2}},
\end{aligned}
$$

the last inequality comes from Proposition 2.12 with $N_{0}=1$. By using (4.7), we obtain

$$
\left\|\langle x\rangle^{-1} f\left(h^{2} P\right) e^{-i t h^{-1}\left(h \Lambda_{g}\right)^{\sigma}} u_{0}\right\|_{L^{p}\left(\mathbb{R}, L^{q}\right)} \leq C h^{-\kappa_{p, q}}\left\|f\left(h^{2} P\right) u_{0}\right\|_{L^{2}} .
$$

This implies that for all $\chi \in C_{0}^{\infty}\left(\mathbb{R}^{d}\right)$,

$$
\left\|\chi f\left(h^{2} P\right) e^{-i t h^{-1}\left(h \Lambda_{g}\right)^{\sigma}} u_{0}\right\|_{L^{p}\left(\mathbb{R}, L^{q}\right)} \leq C h^{-\kappa_{p, q}}\left\|f\left(h^{2} P\right) u_{0}\right\|_{L^{2}} .
$$

Therefore, by scaling in time, we get

$$
\left\|\chi f\left(h^{2} P\right) e^{-i t \Lambda_{g}^{\sigma}} u_{0}\right\|_{L^{p}\left(\mathbb{R}, L^{q}\right)} \leq C h^{-\gamma_{p, q}}\left\|f\left(h^{2} P\right) u_{0}\right\|_{L^{2}} .
$$

The proof of (3.19) is now complete. 


\section{StrichartZ estimates OUtSide COMPaCt Sets}

5.1. The Isozaki-Kitada parametrix.

Notations and the Hamilton-Jacobi equations. For any $J \Subset(0,+\infty)$ an open interval, any $R>0$, any $\tau \in(-1,1)$, we define the outgoing region $\Gamma^{+}(R, J, \tau)$ and the incoming region $\Gamma^{-}(R, J, \tau)$ by

$$
\Gamma^{ \pm}(R, J, \tau):=\left\{(x, \xi) \in \mathbb{R}^{2 d},|x|>R,|\xi|^{2} \in J, \pm \frac{x \cdot \xi}{|x||\xi|}>\tau\right\} .
$$

Let $\sigma \in(0, \infty)^{1}$. We will use the so called Isozaki-Kitada parametrix to give an approximation at high frequency of the form

$$
e^{-i t h^{-1} \psi\left(h^{2} P\right)} O p^{h}\left(\chi^{ \pm}\right)=J_{h}^{ \pm}\left(a^{ \pm}(h)\right) e^{-i t h^{-1}(h \Lambda)^{\sigma}} J_{h}^{ \pm}\left(b^{ \pm}(h)\right)^{\star}+R_{N}^{ \pm}(h),
$$

with $\Lambda=\sqrt{-\Delta}$ where $\Delta$ is the free Laplacian operator on $\mathbb{R}^{d}$ and $\psi(\cdot)=\tilde{f}(\cdot) \sqrt{ }^{-\sigma} \in C_{0}^{\infty}(\mathbb{R} \backslash\{0\})$ for some $\tilde{f} \in C_{0}^{\infty}(\mathbb{R} \backslash\{0\})$ satisfying $\tilde{f}=1$ near $\operatorname{supp}(f)$. The functions $\chi^{ \pm}$are supported in $\Gamma^{ \pm}\left(R^{4}, J_{4}, \tau_{4}\right)$ (see Proposition 5.6 for the choice of $J_{4}$ and $\tau_{4}$ ) and

$$
J_{h}^{ \pm}\left(a^{ \pm}(h)\right)=\sum_{j=1}^{N-1} h^{j} J_{h}^{ \pm}\left(a_{j}^{ \pm}\right),
$$

where

$$
J_{h}^{ \pm}\left(a^{ \pm}\right) u(x)=(2 \pi h)^{-d} \iint_{\mathbb{R}^{2 d}} e^{i h^{-1}\left(S_{R}^{ \pm}(x, \xi)-y \cdot \xi\right)} a^{ \pm}(x, \xi) u(y) d y d \xi, \quad u \in \mathscr{S}\left(\mathbb{R}^{d}\right) .
$$

The amplitude functions $a_{j}^{ \pm}$are supported in $\Gamma^{ \pm}\left(R, J_{1}, \tau_{1}\right)$ (see Proposition 5.1) and the phase functions $S_{R}^{ \pm}:=S_{1, R}^{ \pm}$will be described later. The same notation for $J_{h}^{ \pm}\left(b^{ \pm}(h)\right)$ is used with $b_{k}^{ \pm}$in place of $a_{j}^{ \pm}$.

The Isozaki-Kitada parametrix at low frequency is of the form

$$
e^{-i t \epsilon \psi\left(\epsilon^{-2} P\right)} O p_{\epsilon}\left(\chi_{\epsilon}^{ \pm}\right) \zeta(\epsilon x)=\mathcal{J}_{\epsilon}^{ \pm}\left(a_{\epsilon}^{ \pm}\right) e^{-i t \epsilon \Lambda^{\sigma}} \mathcal{J}_{\epsilon}^{ \pm}\left(b_{\epsilon}^{ \pm}\right)^{\star}+\mathcal{R}_{N}^{ \pm}(t, \epsilon)
$$

where $\psi$ is as above and $\zeta \in C^{\infty}\left(\mathbb{R}^{d}\right)$ supported outside $B(0,1)$ and equal to 1 near infinity. The functions $\chi_{\epsilon}^{ \pm}$are supported in $\Gamma^{ \pm}\left(R^{4}, J_{4}, \tau_{4}\right)$ and

$$
\mathcal{J}_{\epsilon}^{ \pm}\left(a_{\epsilon}^{ \pm}\right)=\sum_{j=1}^{N} \mathcal{J}_{\epsilon}^{ \pm}\left(a_{\epsilon, j}^{ \pm}\right),
$$

where

$$
\mathcal{J}_{\epsilon}^{ \pm}(a):=D_{\epsilon} J_{\epsilon}^{ \pm}(a), \quad \mathcal{J}_{\epsilon}^{ \pm}(b)^{\star}:=J_{\epsilon}^{ \pm}(b)^{\star} D_{\epsilon}^{-1}
$$

with $D_{\epsilon}$ as in (2.4),

$$
J_{\epsilon}^{ \pm}(a) u(x):=(2 \pi)^{-d} \iint_{\mathbb{R}^{2 d}} e^{i\left(S_{\epsilon, R}^{ \pm}(x, \xi)-y \cdot \xi\right)} a(x, \xi) u(y) d y d \xi
$$

and

$$
J_{\epsilon}^{ \pm}(b)^{\star} u(x)=(2 \pi)^{-d} \iint_{\mathbb{R}^{2 d}} e^{i\left(x \cdot \xi-S_{\epsilon, R}^{ \pm}(y, \xi)\right)} \overline{b(y, \xi)} u(y) d y d \xi
$$

The amplitude functions $a_{\epsilon, j}^{ \pm}$are supported in $\Gamma^{ \pm}\left(R, J_{1}, \tau_{1}\right)$ and the phase functions $S_{\epsilon, R}^{ \pm}$will be described in the next proposition. The same notation for $\mathcal{J}_{\epsilon}^{ \pm}\left(b_{\epsilon}^{ \pm}\right)$will be used with $b_{\epsilon}^{ \pm}$in place of $a_{\epsilon}^{ \pm}$.

\footnotetext{
${ }^{1}$ The construction of the Isozaki-Kitada parametrix we present here works well for the (half) wave equation, i.e. $\sigma=1$.
} 
Proposition 5.1. Fix $J_{1} \Subset(0,+\infty)$ and $\tau_{1} \in(-1,1)$. Then there exists two families of smooth functions $\left(S_{\epsilon, R}^{ \pm}\right)_{R \gg 1}$ satisfying the following Hamilton-Jacobi equation

$$
p_{\epsilon}\left(x, \nabla_{x} S_{\epsilon, R}^{ \pm}(x, \xi)\right)=|\xi|^{2},
$$

for all $(x, \xi) \in \Gamma^{ \pm}\left(R, J_{1}, \tau_{1}\right)$, where $p_{\epsilon}$ is given in (2.5). Moreover, for all $\alpha, \beta \in \mathbb{N}^{d}$, there exists $C_{\alpha \beta}>0$ such that

$$
\left|\partial_{x}^{\alpha} \partial_{\xi}^{\beta}\left(S_{\epsilon, R}^{ \pm}(x, \xi)-x \cdot \xi\right)\right| \leq C_{\alpha \beta} \min \left\{R^{1-\rho-|\alpha|},\langle x\rangle^{1-\rho-|\alpha|}\right\},
$$

for all $x, \xi \in \mathbb{R}^{d}$, all $\epsilon \in(0,1]$ and $R \gg 1$.

Remark 5.2. From (5.5), we see that for $R>0$ large enough, the phase functions satisfy for all $x, \xi \in \mathbb{R}^{d}$ and all $\epsilon \in(0,1]$,

$$
\left\|\nabla_{x} \cdot \nabla_{\xi} S_{\epsilon, R}^{ \pm}(x, \xi)-\operatorname{Id}_{\mathbb{R}^{d}}\right\| \leq \frac{1}{2},
$$

and for all $|\alpha| \geq 1$ and all $|\beta| \geq 1$,

$$
\left|\partial_{x}^{\alpha} \partial_{\xi}^{\beta} S_{\epsilon, R}^{ \pm}(x, \xi)\right| \leq C_{\alpha \beta} .
$$

The estimates (5.6) and (5.7) are useful in the construction of Isozaki-Kitada parametrix as well as the $L^{2}$-boundedness of Fourier integral operators.

Proof of Proposition 5.1. We firstly note that the case $\epsilon=1$ is given in [7, Proposition 3.1]. Let $J_{1} \Subset J_{0} \Subset(0,+\infty)$ and $-1<\tau_{0}<\tau_{1}<1$. By using Lemma 2.4 , in the region $\Gamma^{ \pm}\left(R / 2, J_{0}, \tau_{0}\right)$ which implies that $|x|>1$, we see that the function $p_{\epsilon}(x, \xi)$ satisfies for all $\alpha, \beta \in \mathbb{N}^{d}$, there exists $C_{\alpha \beta}>0$ such that for all $(x, \xi) \in \Gamma^{ \pm}\left(R / 2, J_{0}, \tau_{0}\right)$ and all $\epsilon \in(0,1]$,

$$
\left|\partial_{x}^{\alpha} \partial_{\xi}^{\beta} p_{\epsilon}(x, \xi)\right| \leq C_{\alpha \beta}\langle\xi\rangle^{2-|\beta|} .
$$

Thanks to this uniform bound, by using the argument given in [37, Proposition 4.1], we can solve (for $R>0$ large enough) the Hamilton-Jacobi equation (5.4) in $\Gamma^{ \pm}\left(R / 2, J_{0}, \tau_{0}\right)$ uniformly with respect to $\epsilon \in(0,1]$. We denote such solutions by $\tilde{S}_{\epsilon}^{ \pm}$. Next, by choosing a special cutoff (see [7], see also (5.9)) $\chi_{R}^{ \pm} \in S(0,-\infty)$ such that $\chi_{R}^{ \pm}(x, \xi)=1$ for $(x, \xi) \in \Gamma^{ \pm}\left(R, J_{1}, \tau_{1}\right)$ and $\operatorname{supp}\left(\chi_{R}^{ \pm}\right) \subset \Gamma^{ \pm}\left(R / 2, J_{0}, \tau_{0}\right)$, then the functions

$$
S_{\epsilon, R}^{ \pm}(x, \xi)=\chi_{R}^{ \pm}(x, \xi) \tilde{S}_{\epsilon}^{ \pm}(x, \xi)+\left(1-\chi_{R}^{ \pm}\right)(x, \xi)\langle x, \xi\rangle
$$

satisfy the properties of Proposition 5.1, where $\langle x, \xi\rangle=x \cdot \xi$.

Construction of the parametrix. Let us firstly consider the high frequency case (5.1). The construction in the low frequeny case (5.2) is similar up to some modifications (see after Theorem 5.8). We only treat the outgoing case $(+)$, the incoming one is similar. We start with the following Duhamel formula

$$
\begin{aligned}
& e^{-i t h^{-1} \psi\left(h^{2} P\right)} J_{h}^{+}\left(a^{+}(h)\right)=J_{h}^{+}\left(a^{+}(h)\right) e^{-i t h^{-1}(h \Lambda)^{\sigma}} \\
& \quad-i h^{-1} \int_{0}^{t} e^{-i(t-s) h^{-1} \psi\left(h^{2} P\right)}\left(\psi\left(h^{2} P\right) J_{h}^{+}\left(a^{+}(h)\right)-J_{h}^{+}\left(a^{+}(h)\right)(h \Lambda)^{\sigma}\right) e^{-i s h^{-1}(h \Lambda)^{\sigma}} d s .
\end{aligned}
$$

We want the term $\psi\left(h^{2} P\right) J_{h}^{+}\left(a^{+}(h)\right)-J_{h}^{+}\left(a^{+}(h)\right)(h \Lambda)^{\sigma}$ to have a small contribution. To do so, we firstly introduce a special cutoff. For any $J_{2} \Subset J_{1} \Subset(0,+\infty)$ and $-1<\tau_{1}<\tau_{2}<1$, we define

$$
\chi_{1 \rightarrow 2}^{+}(x, \xi)=\kappa\left(\frac{|x|}{R^{2}}\right) \rho_{1 \rightarrow 2}\left(|\xi|^{2}\right) \theta_{1 \rightarrow 2}\left(+\frac{x \cdot \xi}{|x||\xi|}\right),
$$


where $\kappa \in C^{\infty}(\mathbb{R})$ is non-decreasing such that

$$
\kappa(t)=\left\{\begin{array}{ll}
1 & \text { when } t \geq 1 / 2 \\
0 & \text { when } t \leq 1 / 4
\end{array},\right.
$$

and $\rho_{1 \rightarrow 2} \in C^{\infty}(\mathbb{R})$ is non-decreasing such that $\rho_{1 \rightarrow 2}=1$ near $J_{2}$, supported in $J_{1}$ and $\theta_{1 \rightarrow 2} \in C_{0}^{\infty}(\mathbb{R})$ such that

$$
\theta_{1 \rightarrow 2}(t)=\left\{\begin{array}{ll}
1 & \text { when } t>\tau_{2}-\varepsilon \\
0 & \text { when } t<\tau_{1}+\varepsilon
\end{array},\right.
$$

with $\varepsilon \in\left(0, \tau_{2}-\tau_{1}\right)$. We see that $\chi_{1 \rightarrow 2}^{+} \in S(0,-\infty)$ and for $R \gg 1$,

$$
\operatorname{supp}\left(\chi_{1 \rightarrow 2}^{+}\right) \subset \Gamma^{+}\left(R, J_{1}, \tau_{1}\right), \quad \chi_{1 \rightarrow 2}^{+}=1 \text { near } \Gamma^{+}\left(R^{2}, J_{2}, \tau_{2}\right) .
$$

Proposition 5.3. Let $S_{R}^{+}:=S_{1, R}^{+}$be the solution of (5.4) given as in Proposition 5.1. Let $J_{2}$ be an arbitrary open interval such that $J_{2} \Subset J_{1} \Subset(0,+\infty)$ and $\tau_{2}$ be an arbitrary real number such that $-1<\tau_{1}<\tau_{2}<1$. Then for $R>0$ large enough, we can find a sequence of symbols $a_{j}^{+} \in S(-j,-\infty)$ supported in $\Gamma^{+}\left(R, J_{1}, \tau_{1}\right)$ such that for all $N \geq 1$,

$$
\begin{aligned}
\psi\left(h^{2} P\right) J_{h}^{+}\left(a^{+}(h)\right)- & J_{h}^{+}\left(a^{+}(h)\right)(h \Lambda)^{\sigma} \\
\sup _{\Gamma^{+}\left(R, J_{1}, \tau_{1}\right)}\left|a_{0}^{+}(x, \xi)\right| & \gtrsim 1,
\end{aligned}
$$

where $a^{+}(h)=\sum_{j=0}^{N-1} h^{j} a_{j}^{+}$and $\left(r_{N}^{+}(h)\right)_{h \in(0,1]}$ is bounded in $S(-N,-\infty), R_{N}(h)$ is as in Proposition 2.2, $\left(\check{a}^{+}(h)\right)_{h \in(0,1]}$ is bounded in $S(0,-\infty)$ and is a finite sum depending on $N$ of the form

$$
\check{a}^{+}(h)=\sum_{|\alpha| \geq 1} \check{a}_{\alpha}^{+}(h) \partial_{x}^{\alpha} \chi_{1 \rightarrow 2}^{+},
$$

with $\left(\check{a}_{\alpha}^{+}(h)\right)_{h \in(0,1]}$ bounded in $S(0,-\infty)$ and $\chi_{1 \rightarrow 2}^{+}$given in $(5.9)$.

Proof. We firstly use the parametrix of $\psi\left(h^{2} P\right)$ given in Proposition 2.2 and get

$$
\psi\left(h^{2} P\right)=O p^{h}(q(h))+h^{N} R_{N}(h),
$$

where $q(h)=\sum_{k=0}^{N-1} h^{k} q_{k}$ and $q_{k} \in S(-k,-\infty), k=0, \ldots, N-1$. Note that $q_{0}(x, \xi)=\psi(p(x, \xi))=$ $\tilde{f}(p(x, \xi)) \sqrt{p(x, \xi)}$ and $\operatorname{supp}\left(q_{k}\right) \subset \operatorname{supp}(\psi \circ p)$. Up to remainder term, we consider the action of $O p^{h}(q(h))$ on $J_{h}^{+}\left(a^{+}(h)\right)$. To do this, we need the following action of a pseudo-differential operator on a Fourier integral operator (see e.g. [35, Theorem IV-19], [2, Appendix] or [38]).

Proposition 5.4. Let $a \in S\left(\mu_{1},-\infty\right)$ and $b \in S\left(\mu_{2},-\infty\right)$ and $S$ satisfy (5.6) and (5.7). Then

$$
O p^{h}(a) \circ J_{h}(S, b)=\sum_{j=0}^{N-1} h^{j} J_{h}(S,(a \triangleleft b) j)+h^{N} J_{h}\left(S, r_{N}(h)\right),
$$

where $(a \triangleleft b)_{j}$ is an universal linear combination of

$$
\partial_{\xi}^{\beta} a\left(x, \nabla_{x} S(x, \xi)\right) \partial_{x}^{\beta-\alpha} b(x, \xi) \partial_{x}^{\alpha_{1}} S(x, \xi) \cdots \partial_{x}^{\alpha_{k}} S(x, \xi),
$$

with $\alpha \leq \beta, \alpha_{1}+\cdots+\alpha_{k}=\alpha$ and $\left|\alpha_{l}\right| \geq 2$ for all $l=1, \ldots, k$ and $|\beta|=j$. The maps $(a, b) \mapsto$ $(a \triangleleft b)_{j}$ and $(a, b) \mapsto r_{N}(h)$ are continuous from $S\left(\mu_{1},-\infty\right) \times S\left(\mu_{2},-\infty\right)$ to $S\left(\mu_{1}+\mu_{2}-j,-\infty\right)$ and $S\left(\mu_{1}+\mu_{2}-N,-\infty\right)$ respectively. In particular, we have

$$
\begin{aligned}
(a \triangleleft b)_{0}(x, \xi) & =a\left(x, \nabla_{x} S(x, \xi)\right) b(x, \xi), \\
i(a \triangleleft b)_{1}(x, \xi) & =\nabla_{\xi} a\left(x, \nabla_{x} S(x, \xi)\right) \cdot \nabla_{x} b(x, \xi)+\frac{1}{2} \operatorname{tr}\left(\nabla_{\xi}^{2} a\left(x, \nabla_{x} S(x, \xi)\right) \cdot \nabla_{x}^{2} S(x, \xi)\right) b(x, \xi) .
\end{aligned}
$$


Using this result, we have

$$
O p^{h}(q(h)) J_{h}^{+}\left(a^{+}(h)\right)=\sum_{k+j+l=0}^{N-1} h^{k+j+l} J_{h}^{+}\left(\left(q_{k} \triangleleft a_{j}^{+}\right)_{l}\right)+h^{N} J_{h}^{+}\left(r_{N}^{+}(h)\right) .
$$

On the other hand, we have

$$
J_{h}^{+}\left(a^{+}(h)\right)(h \Lambda)^{\sigma}=J_{h}^{+}\left(a^{+}(h)|\xi|^{\sigma}\right) .
$$

Thus we get

$$
\begin{aligned}
\psi\left(h^{2} P\right) J_{h}^{+}\left(a^{+}(h)\right)-J_{h}^{+}\left(a^{+}(h)\right)(h \Lambda)^{\sigma}= & \sum_{r=0}^{N-1} h^{r} J_{h}^{+}\left(\sum_{k+j+l=r}\left(q_{k} \triangleleft a_{j}^{+}\right)_{l}-a_{r}^{+}|\xi|^{\sigma}\right) \\
& +h^{N} J_{h}^{+}\left(r_{N}^{+}(h)\right)+h^{N} R_{N}(h) J_{h}^{+}\left(a^{+}(h)\right) .
\end{aligned}
$$

In order to make the left hand side of (5.10) small, we need to find $a_{j}^{+} \in S(-j,-\infty)$ supported in $\Gamma^{+}\left(R, J_{1}, \tau_{1}\right)$ such that

$$
\sum_{k+j+l=r}\left(q_{k} \triangleleft a_{j}^{+}\right)_{l}-a_{r}^{+}|\xi|^{\sigma}=0, \quad r=0, \ldots, N-1 .
$$

In particular,

$$
\left(q_{0}\left(x, \nabla_{x} S_{R}^{+}(x, \xi)\right)-|\xi|^{\sigma}\right) a_{0}^{+}(x, \xi)=0 .
$$

By noting that if $p(x, \xi) \in \operatorname{supp}(f)$ (see after (5.1)), then $q_{0}(x, \xi)=\sqrt{p(x, \xi)}^{\sigma}$. Thus in the region where the Hamilton-Jacobi equation (5.4) with $\epsilon=1$ is satisfied, we need to show the following transport equations

$$
\begin{aligned}
& \left(q_{0} \triangleleft a_{0}^{+}\right)_{1}+\left(q_{1} \triangleleft a_{0}^{+}\right)_{0}=0 \\
& \left(q_{0} \triangleleft a_{r}^{+}\right)_{1}+\left(q_{1} \triangleleft a_{r}^{+}\right)_{0}=-\sum_{\substack{k+j+l=r+1 \\
j \leq r-1}}\left(q_{k} \triangleleft a_{j}^{+}\right)_{l}, \quad r=1, \ldots, N-1 .
\end{aligned}
$$

Here $\left(q_{0} \triangleleft a^{+}\right)_{1}+\left(q_{1} \triangleleft a^{+}\right)_{0}$ can be written as

$$
i\left[\left(q_{0} \triangleleft a^{+}\right)_{1}(x, \xi)+\left(q_{1} \triangleleft a^{+}\right)_{0}(x, \xi)\right]=\sum_{j=1}^{d} V_{j}^{+}(x, \xi) \partial_{x_{j}} a^{+}(x, \xi)+p_{0}^{+}(x, \xi) a^{+}(x, \xi),
$$

where

$$
\begin{aligned}
V_{j}^{+}(x, \xi) & =\left(\partial_{\xi_{j}} q_{0}\right)\left(x, \nabla_{x} S_{R}^{+}(x, \xi)\right), \\
p_{0}^{+}(x, \xi) & =i q_{1}\left(x, \nabla_{x} S_{R}^{+}(x, \xi)\right)+\frac{1}{2} \operatorname{tr}\left[\nabla_{\xi}^{2} q_{0}\left(x, \nabla_{x} S_{R}^{+}(x, \xi)\right) \cdot \nabla_{x}^{2} S_{R}^{+}(x, \xi)\right] .
\end{aligned}
$$

We now consider the flow $X^{+}(t, x, \xi)$ associated to $V^{+}=\left(V_{j}^{+}\right)_{j=1}^{d}$ as

$$
\left\{\begin{array}{l}
\dot{X}^{+}(t)=V^{+}\left(X^{+}(t), \xi\right) \\
X^{+}(0)=x
\end{array}\right.
$$

We have the following result (see [3, Proposition 3.2] or [2, Appendix]).

Proposition 5.5. Let $\sigma \in(0, \infty), J_{1} \Subset(0,+\infty)$ and $-1<\tau_{1}<1$. There exists $R>0$ large enough and $e_{1}>0$ small enough such that for all $(x, \xi) \in \Gamma^{+}\left(R, J_{1}, \tau_{1}\right)$, the solution $X^{+}(t, x, \xi)$ to (5.16) is defined for all $t \geq 0$ and satisfies

$$
\begin{aligned}
\left|X^{+}(t, x, \xi)\right| & \geq e_{1}(t+|x|), \\
\left(X^{+}(t, x, \xi), \xi\right) & \in \Gamma^{+}\left(R, J_{1}, \tau_{1}\right) .
\end{aligned}
$$


Moreover, for all $\alpha, \beta \in \mathbb{N}^{d}$, there exists $C_{\alpha \beta}>0$ such that for all $t \geq 0$ and all $h \in(0,1]$,

$$
\left|\partial_{x}^{\alpha} \partial_{\xi}^{\beta}\left(X^{+}(t, x, \xi)-x-\sigma t \xi|\xi|^{\sigma-2}\right)\right| \leq C_{\alpha \beta}\langle t\rangle\langle x\rangle^{-\rho-|\alpha|},
$$

for all $(x, \xi) \in \Gamma^{+}\left(R, J_{1}, \tau_{1}\right)$.

Now, we can define for $(x, \xi) \in \Gamma^{+}\left(R, J_{1}, \tau_{1}\right)$ the functions

$$
\begin{aligned}
& A_{0}^{+}(x, \xi)=\exp \left(\int_{0}^{+\infty} p_{0}^{+}\left(X^{+}(t, x, \xi), \xi\right) d t\right) \\
& A_{r}^{+}(x, \xi)=\int_{0}^{+\infty} p_{r}^{+}\left(X^{+}(t, x, \xi), \xi\right) \exp \left(\int_{0}^{t} p_{0}^{+}\left(X^{+}(s, x, \xi), \xi\right) d s\right) d t
\end{aligned}
$$

for $r=1, \ldots, N-1$, where

$$
p_{r}^{+}(x, \xi)=i \sum_{\substack{k+j+l=r+1 \\ j \leq r-1}}\left(q_{k} \triangleleft A_{j}^{+}\right)_{l}(x, \xi) .
$$

Using (5.17) and the fact that $p_{r}^{+} \in S(-1-\rho-r,-\infty)$ for $r=0, \ldots, N-1$, we see that $p_{r}^{+}\left(X^{+}(t, x, \xi)\right)$ are integrable with respect to $t$. Hence $A_{r}^{+}(x, \xi)$ are well-defined. Moreover, we have (see e.g. [3, Proposition 3.1]) that for all $(x, \xi) \in \Gamma^{+}\left(R, J_{1}, \tau_{1}\right)$,

$$
\begin{aligned}
\left|\partial_{x}^{\alpha} \partial_{\xi}^{\beta}\left(A_{0}^{+}(x, \xi)-1\right)\right| & \leq C_{\alpha \beta}\langle x\rangle^{-|\alpha|} \\
\left|\partial_{x}^{\alpha} \partial_{\xi}^{\beta} A_{r}^{+}(x, \xi)\right| & \leq C_{\alpha \beta}\langle x\rangle^{-r-|\alpha|} .
\end{aligned}
$$

We also have that $A_{0}^{+}, A_{r}^{+}$for $r=1, \ldots, N-1$ solve (5.14) and (5.15) respectively in $\Gamma^{+}\left(R, J_{1}, \tau_{1}\right)$. Now, by setting $a_{r}^{+}=\chi_{1 \rightarrow 2}^{+} A_{r}^{+}$(see (5.9)), we see that $a_{r}^{+}$are globally defined on $\mathbb{R}^{2 d}$ and $a_{r}^{+} \in S(-r,-\infty)$. It is easy to see (5.11) from (5.20). We next insert $a^{+}(h)=\sum_{j=1}^{N-1} h^{j} a_{j}^{+}$into the left hand side of (5.10) and get

$$
\begin{aligned}
\psi\left(h^{2} P\right) J_{h}^{+}\left(a^{+}(h)\right)-J_{h}^{+}\left(a^{+}(h)\right)(h \Lambda)^{\sigma}= & \sum_{r=0}^{N-1} h^{r} J_{h}^{+}\left(\sum_{k+j+l=r}\left(q_{k} \triangleleft \chi_{1 \rightarrow 2}^{+} A_{j}^{+}\right)_{l}-\chi_{1 \rightarrow 2}^{+} A_{r}^{+}|\xi|^{\sigma}\right) \\
& +h^{N} J_{h}^{+}\left(r_{N}^{+}(h)\right)+h^{N} R_{N}(h) J_{h}^{+}\left(a^{+}(h)\right) .
\end{aligned}
$$

Using the expression of $(a \triangleleft b)_{l}$ given in Proposition 5.4, we see that

$$
\left(q_{k} \triangleleft \chi_{1 \rightarrow 2}^{+} A_{j}^{+}\right)_{l}=\chi_{1 \rightarrow 2}^{+}\left(q_{k} \triangleleft A_{j}^{+}\right)_{l}+\text { terms in which derivatives fall into } \chi_{1 \rightarrow 2}^{+} .
$$

This gives (5.10) with $\check{a}^{+}(h)$ as in (5.12). The proof is complete.

We now are able to construct the symbols $b_{k}^{+}$, for $k=0, \ldots, N-1$.

Proposition 5.6. Let $J_{3}, J_{4}$ and $\tau_{3}, \tau_{4}$ be such that $J_{4} \Subset J_{3} \Subset J_{2}$ and $-1<\tau_{2}<\tau_{3}<\tau_{4}<1$. Then for $R>0$ large enough and all $\chi^{+}$supported in $\Gamma^{+}\left(R^{4}, J_{4}, \tau_{4}\right)$, there exists a sequence of symbols $b_{k}^{+} \in S(-k,-\infty)$, for $k=0, \ldots, N-1$, supported in $\Gamma^{+}\left(R^{3}, J_{3}, \tau_{3}\right)$ such that

$$
J_{h}^{+}\left(a^{+}(h)\right) J_{h}^{+}\left(b^{+}(h)\right)^{\star}=O p^{h}\left(\chi^{+}\right)+h^{N} O p^{h}\left(\tilde{r}_{N}^{+}(h)\right),
$$

where $a^{+}(h)=\sum_{j=0}^{N-1} h^{j} a_{j}^{+}$is given in Proposition 5.3 and $b^{+}(h)=\sum_{k=0}^{N-1} h^{k} b_{k}^{+}$and $\left(\tilde{r}_{N}^{+}(h)\right)_{h \in(0,1]}$ is bounded in $S(-N,-\infty)$.

Before giving the proof, we need the following result (see [2, Appendix] or [3, Lemma 3.3]). 
Lemma 5.7. Let $S_{R}^{+}:=S_{1, R}^{+}$be as in Proposition 5.1. For $x, y, \xi \in \mathbb{R}^{d}$, we define

$$
\eta^{+}(R, x, y, \xi):=\int_{0}^{1} \nabla_{x} S_{R}^{+}(y+\lambda(x-y), \xi) d \lambda .
$$

Then for $R>0$ large enough, we have the following properties.

i. For all $x, y \in \mathbb{R}^{d}$, the map $\xi \mapsto \eta^{+}(R, x, y, \xi)$ is a diffeomorphism from $\mathbb{R}^{d}$ onto itself. Let $\eta \mapsto \xi^{+}(R, x, y, \eta)$ be its inverse.

ii. There exists $C>1$ such that for all $x, y, \eta \in \mathbb{R}^{d}$,

$$
C^{-1}\langle\eta\rangle \leq\left\langle\xi^{+}(R, x, y, \eta)\right\rangle \leq C\langle\eta\rangle .
$$

iii. For all $\alpha, \alpha^{\prime}, \beta \in \mathbb{N}^{d}$, there exists $C_{\alpha \alpha^{\prime} \beta}>0$ such that for all $x, y, \eta \in \mathbb{R}^{d}$ and all $k \leq|\alpha|, k^{\prime} \leq$ $\left|\alpha^{\prime}\right|$,

$$
\left|\partial_{x}^{\alpha} \partial_{y}^{\alpha^{\prime}} \partial_{\eta}^{\beta}\left(\xi^{+}(R, x, y, \eta)-\eta\right)\right| \leq C_{\alpha \alpha^{\prime} \beta}\langle x\rangle^{-k}\langle y\rangle^{-\rho-k^{\prime}}\langle x-y\rangle^{\rho+k+k^{\prime}} .
$$

Proof of Proposition 5.6. We firstly consider the general term $J_{h}^{+}\left(a^{+}\right) J_{h}^{+}\left(b^{+}\right)^{\star}$ and write its kernel as

$$
K_{h}^{+}(x, y)=(2 \pi h)^{-d} \int_{\mathbb{R}^{d}} e^{i h^{-1}\left(S_{R}^{+}(x, \xi)-S_{R}^{+}(y, \xi)\right)} a^{+}(x, \xi) \overline{b^{+}(y, \xi)} d \xi .
$$

By Taylor's formula, we have

$$
S_{R}^{+}(x, \xi)-S_{R}^{+}(y, \xi)=\left\langle x-y, \eta^{+}(R, x, y, \xi)\right\rangle,
$$

where $\eta^{+}$given in (5.22). By change of variable $\xi \mapsto \xi^{+}(R, x, y, \eta)$, the kernel becomes

$$
K_{h}^{+}(x, y)=(2 \pi h)^{-d} \int_{\mathbb{R}^{d}} e^{i h^{-1}(x-y) \eta} a^{+}\left(x, \xi^{+}(R, x, y, \eta)\right) \overline{b^{+}\left(y, \xi^{+}(R, x, y, \eta)\right)}\left|\operatorname{det} \partial_{\eta} \xi^{+}(R, x, y, \eta)\right| d \eta .
$$

Now, using Lemma (5.7), the symbolic calculus gives

$$
J_{h}^{+}\left(a^{+}\right) J_{h}^{+}\left(b^{+}\right)^{\star}=\sum_{l=0}^{N-1} h^{l} O p^{h}\left(\left(a^{+} \triangleright b^{+}\right)_{l}\right)+h^{N} O p^{h}\left(\tilde{r}_{N}^{+}(h)\right),
$$

where $\left(a^{+} \triangleright b^{+}\right)_{l} \in S(-l,-\infty)$ is of the form

$$
\left(a^{+} \triangleright b^{+}\right)_{l}(x, \eta)=\sum_{|\alpha|=l} \frac{\left.\partial_{y}^{\alpha} D_{\eta}^{\alpha} c^{+}(x, y, \eta)\right|_{y=x}}{\alpha !},
$$

for $l=0, \ldots, N-1$ with

$$
c^{+}(x, y, \eta)=a^{+}\left(x, \xi^{+}(R, x, y, \eta)\right) \overline{b^{+}\left(y, \xi^{+}(R, x, y, \eta)\right)}\left|\operatorname{det} \partial_{\eta} \xi^{+}(R, x, y, \eta)\right|,
$$

and $\left(\tilde{r}_{N}^{+}(h)\right)_{h \in(0,1]}$ is bounded in $S(-N,-\infty)$. We have now

$$
\begin{aligned}
J_{h}^{+}\left(a^{+}(h)\right) J_{h}^{+}\left(b^{+}(h)\right)^{\star} & =\sum_{j, k} h^{j+k} J_{h}^{+}\left(a_{j}^{+}\right) J_{h}^{+}\left(b_{k}^{+}\right)^{\star} \\
& =\sum_{j+k+l=0}^{N-1} h^{j+k+l} O p^{h}\left(\left(a_{j}^{+} \triangleleft b_{k}^{+}\right)_{l}\right)+h^{N} O p^{h}\left(\tilde{r}_{N}^{+}(h)\right) .
\end{aligned}
$$

Compare with (5.21), the result follows if we solve the following equations:

$$
\begin{aligned}
& \left(a_{0}^{+} \triangleleft b_{0}^{+}\right)_{0}=\chi^{+}, \\
& \left(a_{0}^{+} \triangleleft b_{r}^{+}\right)_{0}=-\sum_{\substack{j+k+l=r \\
k \leq r-1}}\left(a_{j}^{+} \triangleleft b_{k}^{+}\right)_{l}, \quad r=1, \ldots, N-1 .
\end{aligned}
$$


We can define $b_{0}^{+}, \ldots, b_{N-1}^{+}$iteratively by

$$
\begin{aligned}
& \overline{b_{0}^{+}(x, \xi)}=\chi^{+}\left(x, \eta^{+}(R, x, x, \xi)\right)\left(a_{0}^{+}(x, \xi)\left|\operatorname{det} \partial_{\eta} \xi^{+}\left(R, x, x, \eta^{+}(R, x, x, \xi)\right)\right|\right)^{-1}, \\
& \overline{b_{r}^{+}(x, \xi)}=-\sum_{\substack{j+k+l=r \\
k \leq r-1}}\left(a_{j}^{+} \triangleleft b_{k}^{+}\right)_{l}\left(x, \eta^{+}(R, x, x, \xi)\right)\left(a_{0}^{+}(x, \xi)\left|\operatorname{det} \partial_{\eta} \xi\left(R, x, x, \eta^{+}(R, x, x, \xi)\right)\right|\right)^{-1},
\end{aligned}
$$

for $r=1, \ldots, N-1$. Note that by (5.11) and Lemma 5.7, the term in $(\cdots)^{-1}$ cannot vanish on the support of $\chi^{+}\left(\cdot, \eta^{+}(R, \cdot, \cdot, \cdot)\right)$. Thus the above functions are well-defined. Moreover, by choosing $R>0$ large enough with the fact

$$
\eta^{+}(R, x, x, \xi)=\nabla_{x} S_{R}^{+}(x, \xi)=\xi+O\left(\min \left\{R^{-\rho},\langle x\rangle^{-\rho}\right\}\right),
$$

we see that the support of $\chi^{+}\left(x, \eta^{+}(R, x, x, \xi)\right)$ is contained in $\Gamma^{+}\left(R^{3}, J_{3}, \tau_{3}\right)$. This completes the proof of Proposition 5.6.

By (5.8), Proposition 5.3 and Proposition 5.6, we are able to state the Isozaki-Kitada parametrix for the fractional Schrödinger equation at high frequency.

Theorem 5.8. Let $\sigma \in(0, \infty)$. Fix $J_{4} \Subset(0,+\infty)$ open interval containing $\operatorname{supp}(f)$ and $-1<\tau_{4}<1$. Choose arbitrary open intervals $J_{1}, J_{2}, J_{3}$ such that $J_{4} \Subset J_{3} \Subset J_{2} \Subset J_{1} \Subset(0,+\infty)$ and arbitrary $\tau_{1}, \tau_{2}, \tau_{3}$ such that $-1<\tau_{1}<\tau_{2}<\tau_{3}<\tau_{4}<1$. Then for $R>0$ large enough, we can find sequences of symbols

such that for all

$$
a_{j}^{ \pm} \in S(-j,-\infty), \quad \operatorname{supp}\left(a_{j}^{ \pm}\right) \subset \Gamma^{ \pm}\left(R, J_{1}, \tau_{1}\right),
$$

there exist sequences of symbols

$$
\chi^{ \pm} \in S(0,-\infty), \quad \operatorname{supp}\left(\chi^{ \pm}\right) \subset \Gamma^{ \pm}\left(R^{4}, J_{4}, \tau_{4}\right),
$$

$$
b_{k}^{ \pm} \in S(-k,-\infty), \quad \operatorname{supp}\left(b_{k}^{ \pm}\right) \subset \Gamma^{ \pm}\left(R^{3}, J_{3}, \tau_{3}\right),
$$

such that for all $N \geq 1$, for all $h \in(0,1]$ and all $\pm t \geq 0$,

$$
e^{-i t h^{-1} \psi\left(h^{2} P\right)} O p^{h}\left(\chi^{ \pm}\right)=J_{h}^{ \pm}\left(a^{ \pm}(h)\right) e^{-i t h^{-1}(h \Lambda)^{\sigma}} J_{h}^{ \pm}\left(b^{ \pm}(h)\right)^{\star}+R_{N}^{ \pm}(t, h),
$$

where the phase functions $S_{R}^{ \pm}:=S_{1, R}^{ \pm}$are as in Proposition 5.1 and the remainder terms

$$
R_{N}^{ \pm}(t, h)=R_{1}^{ \pm}(N, t, h)+R_{2}^{ \pm}(N, t, h)+R_{3}^{ \pm}(N, t, h)+R_{4}^{ \pm}(N, t, h),
$$

with

$$
\begin{aligned}
& R_{1}^{ \pm}(N, t, h)=-h^{N-1} e^{-i t h^{-1} \psi\left(h^{2} P\right)} O p^{h}\left(\tilde{r}_{N}^{ \pm}(h)\right), \\
& R_{2}^{ \pm}(N, t, h)=-i h^{N-1} \int_{0}^{t} e^{-i(t-s) h^{-1} \psi\left(h^{2} P\right)} R_{N}(h) J_{h}^{ \pm}\left(a^{ \pm}(h)\right) e^{-i s h^{-1}(h \Lambda)^{\sigma}} J_{h}^{ \pm}\left(b^{ \pm}(h)\right)^{\star} d s, \\
& R_{3}^{ \pm}(N, t, h)=-i h^{N-1} \int_{0}^{t} e^{-i(t-s) h^{-1} \psi\left(h^{2} P\right)} J_{h}^{ \pm}\left(r_{N}^{ \pm}(h)\right) e^{-i s h^{-1}(h \Lambda)^{\sigma}} J_{h}^{ \pm}\left(b^{ \pm}(h)\right)^{\star} d s, \\
& R_{4}^{ \pm}(N, t, h)=-i h^{-1} \int_{0}^{t} e^{-i(t-s) h^{-1} \psi\left(h^{2} P\right)} J_{h}^{ \pm}\left(\check{a}^{ \pm}(h)\right) e^{-i s h^{-1}(h \Lambda)^{\sigma}} J_{h}^{ \pm}\left(b^{ \pm}(h)\right)^{\star} d s .
\end{aligned}
$$

Here $\left(\tilde{r}_{N}^{ \pm}(h)\right)_{h \in(0,1]},\left(r_{N}^{ \pm}(h)\right)_{h \in(0,1]}$ are bounded in $S(-N,-\infty), R_{N}(h)$ is as in $(5.13),\left(\check{a}^{ \pm}(h)\right)_{h \in(0,1]}$ are bounded in $S(0,-\infty)$ and are finite sums depending on $N$ of the form

$$
\check{a}^{ \pm}(h)=\sum_{|\alpha| \geq 1} \check{a}_{\alpha}^{ \pm}(h) \partial_{x}^{\alpha} \chi_{1 \rightarrow 2}^{ \pm},
$$

where $\left(\check{a}_{\alpha}^{ \pm}(h)\right)_{h \in(0,1]}$ are bounded in $S(0,-\infty)$ and $\chi_{1 \rightarrow 2}^{ \pm}$are given in $(5.9)$. 
We now give the main steps for the construction of the Isozaki-Kitada parametrix at low frequency. For simplicity, we omit the \pm sign. Let us start with the following Duhamel formula

$$
e^{-i t \epsilon \psi\left(\epsilon^{-2} P\right)} \mathcal{J}_{\epsilon}\left(a_{\epsilon}\right)=\mathcal{J}_{\epsilon}\left(a_{\epsilon}\right) e^{-i t \epsilon \Lambda^{\sigma}}-i \epsilon \int_{0}^{t} e^{-i(t-s) \epsilon \psi\left(\epsilon^{-2} P\right)}\left(\psi\left(\epsilon^{-2} P\right) \mathcal{J}_{\epsilon}\left(a_{\epsilon}\right)-\mathcal{J}_{\epsilon}\left(a_{\epsilon}\right) \Lambda^{\sigma}\right) e^{-i s \epsilon \Lambda^{\sigma}} d s .
$$

Thanks to the support of $a_{\epsilon}$, we can write

$$
\psi\left(\epsilon^{-2} P\right) \mathcal{J}_{\epsilon}\left(a_{\epsilon}\right)=\psi\left(\epsilon^{-2} P\right) \zeta_{1}(\epsilon x) \mathcal{J}_{\epsilon}\left(a_{\epsilon}\right),
$$

where $\zeta_{1} \in C^{\infty}\left(\mathbb{R}^{d}\right)$ is supported outside $B(0,1)$ and satisfies $\zeta_{1}(x)=1$ for $|x|>R$. Using the parametrix of $\psi\left(\epsilon^{-2} P\right) \zeta_{1}(\epsilon x)$ given in Proposition 2.7 (by taking the adjoint), we have

$$
\psi\left(\epsilon^{-2} P\right) \zeta_{1}(\epsilon x)=\sum_{k=0}^{N-1} \tilde{\zeta}_{1}(\epsilon x) O p_{\epsilon}\left(q_{\epsilon, k}\right) \zeta_{1}(\epsilon x)+R_{N}(\epsilon)
$$

where $q_{\epsilon, 0}(x, \xi)=\psi\left(p_{\epsilon}(x, \xi)\right)=\tilde{f}\left(p_{\epsilon}(x, \xi)\right){\sqrt{p_{\epsilon}(x, \xi)}}^{\sigma}, \operatorname{supp}\left(q_{\epsilon, k}\right) \subset \operatorname{supp}\left(\psi \circ p_{\epsilon}\right)$ and $\left(R_{N}(\epsilon)\right)_{\epsilon \in(0,1]}$ satisfies (2.8). Here $\tilde{\zeta}_{1} \in C^{\infty}\left(\mathbb{R}^{d}\right)$ is supported outside $B(0,1)$ and $\tilde{\zeta}_{1}=1$ near $\operatorname{supp}\left(\zeta_{1}\right)$. We want to find $a_{\epsilon}=\sum_{j=0}^{N-1} a_{\epsilon, j}$ so that the term $\psi\left(\epsilon^{-2} P\right) \mathcal{J}_{\epsilon}\left(a_{\epsilon}\right)-\mathcal{J}_{\epsilon}\left(a_{\epsilon}\right) \Lambda^{\sigma}$ has a small contribution. By the choice of cutoff functions and the action of pseudo-differential operators on Fourier integral operators given in Proposition 5.4 with $h=1$, we have

$$
\begin{aligned}
\psi\left(\epsilon^{-2} P\right) \mathcal{J}_{\epsilon}\left(a_{\epsilon}\right)-\mathcal{J}_{\epsilon}\left(a_{\epsilon}\right) \Lambda^{\sigma}= & \sum_{r=0}^{N-1}\left(\sum_{k+j+l=r} \mathcal{J}_{\epsilon}\left(\left(q_{\epsilon, k} \triangleleft a_{\epsilon, j}\right)_{l}\right)-\mathcal{J}_{\epsilon}\left(a_{\epsilon, r}|\xi|^{\sigma}\right)\right) \\
& +R_{N}(\epsilon) \mathcal{J}_{\epsilon}\left(a_{\epsilon}\right)+\mathcal{J}_{\epsilon}\left(r_{N}(\epsilon)\right),
\end{aligned}
$$

where $\left(r_{N}(\epsilon)\right)_{\epsilon \in(0,1]}$ is bounded in $S(-N,-\infty)$. This implies that we need to find $\left(a_{\epsilon, j}\right)_{\epsilon \in(0,1]}$ bounded in $S(-j,-\infty)$ supported in $\Gamma\left(R, J_{1}, \tau_{1}\right)$ such that

$$
\sum_{k+j+l=r}\left(q_{\epsilon, k} \triangleleft a_{\epsilon, j}\right)_{l}-a_{\epsilon, r}|\xi|^{\sigma}=0, \quad r=0, \ldots, N-1 .
$$

By noting that if $p_{\epsilon}(x, \xi) \in \operatorname{supp}(f)$, then $q_{\epsilon, 0}(x, \xi)={\sqrt{p_{\epsilon}(x, \xi)}}^{\sigma}$. This leads to the following Hamilton-Jacobi and transport equations,

$$
\begin{aligned}
p_{\epsilon}\left(x, \nabla_{x} S_{\epsilon, R}(x, \xi)\right) & =|\xi|^{2}, \\
\left(q_{\epsilon, 0} \triangleleft a_{\epsilon, 0}\right)_{1}+\left(q_{\epsilon, 1} \triangleleft a_{\epsilon, 0}\right)_{0} & =0 \\
\left(q_{\epsilon, 0} \triangleleft a_{\epsilon, r}\right)_{1}+\left(q_{\epsilon, 1} \triangleleft a_{\epsilon, r}\right)_{0} & =-\sum_{\substack{k+j+l=r+1 \\
j \leq r-1}}\left(q_{\epsilon, k} \triangleleft a_{\epsilon, j}\right)_{l}, \quad r=1, \ldots, N-1 .
\end{aligned}
$$

We can solve (5.25) on $\Gamma^{ \pm}\left(R, J_{1}, \tau_{1}\right)$ using Proposition 5.1. We then solve (5.26), (5.27) on $\Gamma^{ \pm}\left(R, J_{1}, \tau_{1}\right)$ and extend solutions globally on $\mathbb{R}^{2 d}$. We obtain

$$
\psi\left(\epsilon^{-2} P\right) \mathcal{J}_{\epsilon}\left(a_{\epsilon}\right)-\mathcal{J}_{\epsilon}\left(a_{\epsilon}\right) \Lambda^{\sigma}=R_{N}(\epsilon) \mathcal{J}_{\epsilon}\left(a_{\epsilon}\right)+\mathcal{J}_{\epsilon}\left(r_{N}(\epsilon)\right)+\mathcal{J}_{\epsilon}(\check{a}(\epsilon)),
$$

where $(\check{a}(\epsilon))_{\epsilon \in(0,1]}$ is bounded in $S(0,-\infty)$ and is a finite sum depending on $N$ of the form

$$
\check{a}(\epsilon)=\sum_{|\alpha| \geq 1} \check{a}_{\alpha}(\epsilon) \partial_{x}^{\alpha} \chi_{1 \rightarrow 2},
$$

with $\left(\check{a}_{\alpha}(\epsilon)\right)_{\epsilon \in(0,1]}$ bounded in $S(0,-\infty)$ and $\chi_{1 \rightarrow 2}$ as in $(5.9)$.

Next, we can find bounded families of symbols $b_{\epsilon, k} \in S(-k,-\infty)$ for $k=0, \ldots, N-1$ supported in $\Gamma\left(R^{3}, J_{3}, \tau_{3}\right)$ such that

$$
\mathcal{J}_{\epsilon}\left(a_{\epsilon}\right) \mathcal{J}_{\epsilon}\left(b_{\epsilon}\right)^{\star}=O p_{\epsilon}\left(\chi_{\epsilon}\right) \zeta(\epsilon x)+O p_{\epsilon}\left(\tilde{r}_{N}(\epsilon)\right) \zeta(\epsilon x)
$$


where $b_{\epsilon}=\sum_{k=0}^{N-1} b_{\epsilon, k}$ and $\left(\tilde{r}_{N}(\epsilon)\right)_{\epsilon \in(0,1]}$ is bounded in $S(-N,-\infty)$. This is possible by writing for $R$ large enough $\mathcal{J}_{\epsilon}\left(b_{\epsilon}\right)=\zeta(\epsilon x) \mathcal{J}_{\epsilon}\left(b_{\epsilon}\right)$ and taking the adjoint. We have the following Isozaki-Kitata parametrix for the fractional Schrödinger equation at low frequency.

Theorem 5.9. Let $\sigma \in(0, \infty), \zeta \in C^{\infty}\left(\mathbb{R}^{d}\right)$ be supported outside $B(0,1)$ and equal to 1 near infinity. Fix $J_{4} \Subset(0,+\infty)$ open interval containing $\operatorname{supp}(f)$ and $-1<\tau_{4}<1$. Choose arbitrary open intervals $J_{1}, J_{2}, J_{3}$ such that $J_{4} \Subset J_{3} \Subset J_{2} \Subset J_{1} \Subset(0,+\infty)$ and arbitrary $\tau_{1}, \tau_{2}, \tau_{3}$ such that $-1<\tau_{1}<\tau_{2}<$ $\tau_{3}<\tau_{4}<1$. Then for $R>0$ large enough, we can find bounded families of symbols

$$
\left(a_{\epsilon, j}^{ \pm}\right)_{\epsilon \in(0,1]} \in S(-j,-\infty), \quad \operatorname{supp}\left(a_{\epsilon, j}^{ \pm}\right) \subset \Gamma^{ \pm}\left(R, J_{1}, \tau_{1}\right),
$$

such that for all

there exists families of symbols

$$
\left(\chi_{\epsilon}^{ \pm}\right)_{\epsilon \in(0,1]} \in S(0,-\infty), \quad \operatorname{supp}\left(\chi_{\epsilon}^{ \pm}\right) \subset \Gamma^{ \pm}\left(R^{4}, J_{4}, \tau_{4}\right)
$$

$$
\left(b_{\epsilon, k}^{ \pm}\right)_{\epsilon \in(0,1]} \in S(-k,-\infty), \quad \operatorname{supp}\left(b_{\epsilon, k}^{ \pm}\right) \subset \Gamma^{ \pm}\left(R^{3}, J_{3}, \tau_{3}\right),
$$

such that for all $N \geq 1$, for all $\epsilon \in(0,1]$ and all $\pm t \geq 0$,

$$
e^{-i t \epsilon \psi\left(\epsilon^{-2} P\right)} O p_{\epsilon}\left(\chi_{\epsilon}^{ \pm}\right) \zeta(\epsilon x)=\mathcal{J}_{\epsilon}^{ \pm}\left(a_{\epsilon}^{ \pm}\right) e^{-i t \epsilon \Lambda^{\sigma}} \mathcal{J}_{\epsilon}^{ \pm}\left(b_{\epsilon}^{ \pm}\right)^{\star}+\mathcal{R}_{N}^{ \pm}(t, \epsilon),
$$

where the phase functions $S_{\epsilon, R}^{ \pm}$are given in Proposition 5.1 and the remainder terms

$$
\mathcal{R}_{N}^{ \pm}(t, \epsilon)=\mathcal{R}_{1}^{ \pm}(N, t, \epsilon)+\mathcal{R}_{2}^{ \pm}(N, t, \epsilon)+\mathcal{R}_{3}^{ \pm}(N, t, \epsilon)+\mathcal{R}_{4}^{ \pm}(N, t, \epsilon),
$$

with

$$
\begin{aligned}
& \mathcal{R}_{1}^{ \pm}(N, t, \epsilon)=-e^{-i t \epsilon \psi\left(\epsilon^{-2} P\right)} O p_{\epsilon}\left(\tilde{r}_{N}^{ \pm}(\epsilon)\right) \zeta(\epsilon x) \\
& \mathcal{R}_{2}^{ \pm}(N, t, \epsilon)=-i \epsilon \int_{0}^{t} e^{-i(t-s) \epsilon \psi\left(\epsilon^{-2} P\right)} R_{N}(\epsilon) \mathcal{J}_{\epsilon}^{ \pm}\left(a_{\epsilon}^{ \pm}\right) e^{-i s \epsilon \Lambda^{\sigma}} \mathcal{J}_{\epsilon}^{ \pm}\left(b_{\epsilon}^{ \pm}\right)^{\star} d s \\
& \mathcal{R}_{3}^{ \pm}(N, t, \epsilon)=-i \epsilon \int_{0}^{t} e^{-i(t-s) \epsilon \psi\left(\epsilon^{-2} P\right)} \mathcal{J}_{\epsilon}^{ \pm}\left(r_{N}^{ \pm}(\epsilon)\right) e^{-i s \epsilon \Lambda^{\sigma}} \mathcal{J}_{\epsilon}^{ \pm}\left(b_{\epsilon}^{ \pm}\right)^{\star} d s \\
& \mathcal{R}_{4}^{ \pm}(N, t, \epsilon)=-i \epsilon \int_{0}^{t} e^{-i(t-s) \epsilon \psi\left(\epsilon^{-2} P\right)} \mathcal{J}_{\epsilon}^{ \pm}\left(\check{a}^{ \pm}(\epsilon)\right) e^{-i s \epsilon \Lambda^{\sigma}} \mathcal{J}_{\epsilon}^{ \pm}\left(b_{\epsilon}^{ \pm}\right)^{\star} d s
\end{aligned}
$$

Here $\left(\tilde{r}_{N}^{ \pm}(\epsilon)\right)_{\epsilon \in(0,1]},\left(r_{N}^{ \pm}(\epsilon)\right)_{\epsilon \in(0,1]}$ are bounded in $S(-N,-\infty),\left(R_{N}(\epsilon)\right)_{\epsilon \in(0,1]}$ is given in Proposition 2.7, $\left(\check{a}^{ \pm}(\epsilon)\right)_{\epsilon \in(0,1]}$ are bounded in $S(0,-\infty)$ and are finite sums depending on $N$ of the form

$$
\check{a}^{ \pm}(\epsilon)=\sum_{|\alpha| \geq 1} \check{a}_{\alpha}^{ \pm}(\epsilon) \partial_{x}^{\alpha} \chi_{1 \rightarrow 2}^{ \pm}
$$

where $\left(\check{a}_{\alpha}^{ \pm}(\epsilon)\right)_{\epsilon \in(0,1]}$ are bounded in $S(0,-\infty)$ and $\chi_{1 \rightarrow 2}^{ \pm}$are as in $(5.9)$.

We have the following dispersive estimates for the main terms of the Isozaki-Kitada parametrix both at high and low frequencies.

Proposition 5.10. Let $\sigma \in(0, \infty) \backslash\{1\}, S_{\epsilon, R}^{ \pm}$be as in Proposition 5.1 and $\left(a_{\epsilon}^{ \pm}\right)_{\epsilon \in(0,1]},\left(b_{\epsilon}^{ \pm}\right)_{\epsilon \in(0,1]}$ be bounded in $S(0,-\infty)$ compactly supported in $\xi$ away from zero.

1. Then for $R>0$ large enough, there exists $C>0$ such that for all $t \in \mathbb{R}$ and all $h \in(0,1]$,

$$
\left\|J_{h}^{ \pm}\left(a^{ \pm}\right) e^{-i t h^{-1}(h \Lambda)^{\sigma}} J_{h}^{ \pm}\left(b^{ \pm}\right)^{\star}\right\|_{\mathcal{L}\left(L^{1}, L^{\infty}\right)} \leq C h^{-d}\left(1+|t| h^{-1}\right)^{-d / 2},
$$

where $a^{ \pm}:=a_{\epsilon=1}^{ \pm}, b^{ \pm}:=b_{\epsilon=1}^{ \pm}$.

2. Then for $R>0$ large enough, there exists $C>0$ such that for all $t \in \mathbb{R}$ and all $\epsilon \in(0,1]$,

$$
\left\|\mathcal{J}_{\epsilon}^{ \pm}\left(a_{\epsilon}^{ \pm}\right) e^{-i t \epsilon \Lambda^{\sigma}} \mathcal{J}_{\epsilon}^{ \pm}\left(b_{\epsilon}^{ \pm}\right)^{\star}\right\|_{\mathcal{L}\left(L^{1}, L^{\infty}\right)} \leq C \epsilon^{d}(1+\epsilon|t|)^{-d / 2} .
$$


Proof. 1. For simplicity, we drop the superscript \pm . The kernel of $J_{h}(a) e^{-i t h^{-1}(h \Lambda)^{\sigma}} J_{h}(b)^{\star}$ reads

$$
K_{h}(t, x, y)=(2 \pi h)^{-d} \int_{\mathbb{R}^{d}} e^{i h^{-1}\left(S_{R}(x, \xi)-S_{R}(y, \xi)-t|\xi|^{\sigma}\right)} a(x, \xi) \overline{b(y, \xi)} d \xi .
$$

The estimates (5.28) are in turn equivalent to

$$
\left|K_{h}(t, x, y)\right| \leq C h^{-d}\left(1+|t| h^{-1}\right)^{-d / 2},
$$

for all $t \in \mathbb{R}, h \in(0,1]$ and $x, y \in \mathbb{R}^{d}$. We only consider $t \geq 0$, the case $t \leq 0$ is similar. Let us denote the compact support of the amplitude by $\mathcal{K}$. Since $a, b$ are bounded uniformly in $x, y \in \mathbb{R}^{d}$, we have

$$
\left|K_{h}(t, x, y)\right| \leq C h^{-d}
$$

for all $t \in \mathbb{R}$ and all $x, y \in \mathbb{R}^{d}$. If $0 \leq t \leq h$ or $1+t h^{-1} \leq 2$, then

$$
\left|K_{h}(t, x, y)\right| \leq C h^{-d} \leq C h^{-d}\left(1+t h^{-1}\right)^{-d / 2} .
$$

So, we can assume that $t \geq h$ or $\left(1+t h^{-1}\right) \leq 2 t h^{-1}$ and denote the phase function

$$
\Phi(R, t, x, y, \xi)=\left(S_{R}(x, \xi)-S_{R}(y, \xi)\right) / t-|\xi|^{\sigma},
$$

and parameter $\lambda=t h^{-1} \geq 1$. We can rewrite

$$
\Phi(R, t, x, y, \xi)=\langle(x-y) / t, \eta(R, x, y, \xi)\rangle-|\xi|^{\sigma},
$$

where

$$
\eta(R, x, y, \xi)=\int_{0}^{1} \nabla_{x} S_{R}(y+\lambda(x-y), \xi) d \lambda .
$$

Using the properties of the phase functions $S_{R}$ given in (5.5), we have that

$$
\eta(R, x, y, \xi)=\xi+Q(R, x, y, \xi),
$$

where $Q(R, x, y, \xi)$ is a vector in $\mathbb{R}^{d}$ satisfying for $R>0$ large enough,

$$
\left|\partial_{\xi}^{\beta} Q(R, x, y, \xi)\right| \leq C_{\beta} R^{-\rho},
$$

for all $x, y \in \mathbb{R}^{d}$ and $\xi \in \mathcal{K}$. We have

$$
\nabla_{\xi} \Phi(R, t, x, y, \xi)=\frac{x-y}{t} \cdot\left(\operatorname{Id}_{\mathbb{R}^{d}}+\nabla_{\xi} Q(R, x, y, \xi)\right)-\sigma \xi|\xi|^{\sigma-2} .
$$

If $|(x-y) / t| \geq C$ for some constant $C>0$ large enough then for $R>0$ large enough, there exists $C_{1}>0$,

$$
\left|\nabla_{\xi} \Phi(R, t, x, y, \xi)\right| \geq \frac{1}{2}\left|\frac{x-y}{t}\right| \geq C_{1} .
$$

Thus the phase is non-stationary. By using integration by parts with respect to $\xi$ together with the fact

$$
\left|\partial_{\xi}^{\beta} \Phi(R, t, x, y, \xi)\right| \leq C_{\beta}\left|\frac{x-y}{t}\right|, \quad|\beta| \geq 2,
$$

we have that for all $N \geq 1$,

$$
\left|K_{h}(t, x, y)\right| \leq C h^{-d}\left(t h^{-1}\right)^{-N} \leq C h^{-d}\left(1+t h^{-1}\right)^{-d / 2},
$$

provided $N$ is taken bigger than $d / 2$. The same result still holds for $|(x-y) / t| \leq c$ for some $c>0$ small enough.

Therefore, we can assume that $c \leq|x-y / t| \leq C$. In this case, we write

$$
\nabla_{\xi}^{2} \Phi(R, t, x, y, \xi)=\frac{x-y}{t} \cdot \nabla_{\xi}^{2} Q(R, x, y, \xi)-\sigma|\eta|^{\sigma-2}\left(\operatorname{Id}_{\mathbb{R}^{d}}+(\sigma-2) \frac{\eta \cdot \eta^{t}}{|\eta|^{2}}\right) .
$$


Using the fact that $\sigma \in(0, \infty) \backslash\{1\}$ and

$$
\left.|\operatorname{det} \sigma| \eta\right|^{\sigma-2}\left(\operatorname{Id}_{\mathbb{R}^{d}}+(\sigma-2) \frac{\eta \cdot \eta^{t}}{|\eta|^{2}}\right)\left|=\sigma^{d}\right| \sigma-1 \|\left.\eta\right|^{(\sigma-2) d} \geq C
$$

and (5.31), we see that for $R>0$ large enough, the map $\xi \mapsto \nabla_{\xi} \Phi(R, t, x, y, \xi)$ is a local diffeomorphism from a neighborhood of $\mathcal{K}$ to its range. Moreover, for all $\beta \in \mathbb{N}^{d}$ satisfying $|\beta| \geq 1$, we have $\left|\partial_{\xi}^{\beta} \Phi(R, t, x, y, \xi)\right| \leq C_{\beta}$. The stationary phase theorem then implies that for $R>0$ large enough, all $t \geq h$ and all $x, y \in \mathbb{R}^{d}$ satisfying $c \leq|(x-y) / t| \leq C$,

$$
\left|K_{h}(t, x, y)\right| \leq C h^{-d} \lambda^{-d / 2} \leq C h^{-d}\left(1+t h^{-1}\right)^{-d / 2} .
$$

This gives (5.30).

2. We are now in position to show (5.29). As above, we drop the superscript \pm for simplicity. We see that up to a conjugation by $D_{\epsilon}$, the kernel of $\mathcal{J}_{\epsilon}\left(a_{\epsilon}\right) e^{-i t \epsilon \Lambda^{\sigma}} \mathcal{J}_{\epsilon}\left(b_{\epsilon}\right)^{\star}$ reads

$$
K_{\epsilon}(t, x, y)=(2 \pi)^{-d} \int_{\mathbb{R}^{d}} e^{i\left(S_{\epsilon, R}(x, \xi)-t \epsilon|\xi|^{\sigma}-S_{\epsilon, R}(y, \xi)\right)} a_{\epsilon}(x, \xi) \overline{b_{\epsilon}(y, \xi)} d \xi .
$$

The dispersive estimates (5.29) follow from

$$
\left|K_{\epsilon}(t, x, y)\right| \leq C(1+\epsilon|t|)^{-d / 2}
$$

for all $t \in \mathbb{R}$ uniformly in $x, y \in \mathbb{R}^{d}, \epsilon \in(0,1]$ and the fact that

$$
\left\|D_{\epsilon}\right\|_{\mathcal{L}\left(L^{\infty}\right)}=\epsilon^{d / 2}, \quad\left\|D_{\epsilon}^{-1}\right\|_{\mathcal{L}\left(L^{1}\right)}=\epsilon^{d / 2} .
$$

The estimates (5.32) are proved by repeating the same line as above. The proof is complete.

Micro-local propagation estimates. In this paragraph, we will prove some propagation estimates which are useful for our purpose. To do this, we need the following result (see [8, Lemma 4.1]).

Lemma 5.11. Let $\tau_{+}, \tau_{-} \in(-1,1)$.

1. For all $x, y, \xi \in \mathbb{R}^{d} \backslash\{0\}$ satisfying $\pm x \cdot \xi /|x \| \xi|>\tau_{ \pm}$and $\pm t \geq 0$, we have

$$
\pm \frac{(x+t \xi) \cdot \xi}{|x+t \xi||\xi|}>\tau_{ \pm} \text {and }|x+t \xi| \geq c_{ \pm}(|x|+|t \xi|)
$$

where $c_{ \pm}=\sqrt{1+\tau_{ \pm}} / \sqrt{2}$.

2. If $\tau_{-}+\tau_{+}>0$, then there exists $c=c\left(\tau_{-}, \tau_{+}\right)>0$ such that for all $x, y, \xi \in \mathbb{R}^{d} \backslash\{0\}$ satisfying $+x \cdot \xi /|x \| \xi|>\tau_{+}$and $-y \cdot \xi /|y \| \xi|>\tau_{-}$, we have

$$
|x-y| \geq c(|x|+|y|) \text {. }
$$

We start with the following estimates.

Lemma 5.12. Let $\sigma \in(0, \infty)$ and $\chi \in C_{0}^{\infty}\left(\mathbb{R}^{d}\right)$ satisfying $\chi(x)=1$ for $|x| \leq 1$.

1. Using the notations given in Theorem 5.8, if $R>0$ is large enough, then for all $m \geq 0$, there exists $C>0$ such that for all $\pm s \geq 0$ and all $h \in(0,1]$,

$$
\left\|\chi\left(x / R^{2}\right) J_{h}^{ \pm}\left(\check{a}^{ \pm}(h)\right) e^{-i s h^{-1}(h \Lambda)^{\sigma}} J_{h}^{ \pm}\left(b^{ \pm}(h)\right)^{\star}\langle x\rangle^{m}\right\|_{\mathcal{L}\left(H^{-m}, H^{m}\right)} \leq C h^{m}\langle s\rangle^{-m} .
$$

Moreover,

$$
\left\|\langle x\rangle^{m}(1-\chi)\left(x / R^{2}\right) J_{h}^{ \pm}\left(\check{a}^{ \pm}(h)\right) e^{-i s h^{-1}(h \Lambda)^{\sigma}} J_{h}^{ \pm}\left(b^{ \pm}(h)\right)^{\star}\langle x\rangle^{m}\right\|_{\mathcal{L}\left(H^{-m}, H^{m}\right)} \leq C h^{m}\langle s\rangle^{-m} .
$$

In particular

$$
\left\|\langle x\rangle^{m} J_{h}^{ \pm}\left(\check{a}^{ \pm}(h)\right) e^{-i s h^{-1}(h \Lambda)^{\sigma}} J_{h}^{ \pm}\left(b^{ \pm}(h)\right)^{\star}\langle x\rangle^{m}\right\|_{\mathcal{L}\left(H^{-m}, H^{m}\right)} \leq C h^{m}\langle s\rangle^{-m} .
$$


2. Using the notations given in Theorem 5.9, if $R>0$ is large enough, then for all $m \geq 0$, there exists $C>0$ such that for all $\pm s \geq 0$ and all $\epsilon \in(0,1]$,

$$
\left\|\chi\left(\epsilon x / R^{2}\right) \mathcal{J}_{\epsilon}^{ \pm}\left(\check{a}^{ \pm}(\epsilon)\right) e^{-i s \epsilon \Lambda^{\sigma}} \mathcal{J}_{\epsilon}^{ \pm}\left(b_{\epsilon}^{ \pm}\right)^{\star}\langle\epsilon x\rangle^{m}\right\|_{\mathcal{L}\left(L^{2}\right)} \leq C\langle\epsilon s\rangle^{-m} .
$$

Moreover,

$$
\left\|\langle\epsilon x\rangle^{m}(1-\chi)\left(\epsilon x / R^{2}\right) \mathcal{J}_{\epsilon}^{ \pm}\left(\check{a}^{ \pm}(\epsilon)\right) e^{-i s \epsilon \Lambda^{\sigma}} \mathcal{J}_{\epsilon}^{ \pm}\left(b_{\epsilon}^{ \pm}\right)^{\star}\langle\epsilon x\rangle^{m}\right\|_{\mathcal{L}\left(L^{2}\right)} \leq C\langle\epsilon s\rangle^{-m} .
$$

In particular

$$
\left\|\langle\epsilon x\rangle^{m} \mathcal{J}_{\epsilon}^{ \pm}\left(\check{a}^{ \pm}(\epsilon)\right) e^{-i s \epsilon \Lambda^{\sigma}} \mathcal{J}_{\epsilon}^{ \pm}\left(b_{\epsilon}^{ \pm}\right)^{\star}\langle\epsilon x\rangle^{m}\right\|_{\mathcal{L}\left(L^{2}\right)} \leq C\langle\epsilon s\rangle^{-m} .
$$

Proof. 1. We firstly consider the high frequency case. The proof in this case is essentially given in [8]. For reader's convenience, we will give a sketch of the proof. The kernel of the operator in the left hand side of $(5.35)$ reads

$$
K_{h}^{ \pm}(s, x, y)=(2 \pi h)^{-d} \chi\left(x / R^{2}\right) \int_{\mathbb{R}^{d}} e^{i h^{-1} \Phi^{ \pm}(R, s, x, y, \xi)} \check{a}^{ \pm}(h, x, \xi) \overline{b^{ \pm}(h, y, \xi)} d \xi\langle y\rangle^{m},
$$

where the phase $\Phi^{ \pm}(R, s, x, y, \xi)=S_{R}^{ \pm}(x, \xi)-s|\xi|^{\sigma}-S_{R}^{ \pm}(y, \xi)$. Using (5.5), we have

$$
\left|\nabla_{\xi} \Phi^{ \pm}(R, s, x, y, \xi)\right|=\left.|x-\sigma s \xi| \xi\right|^{\sigma-2}-y+O(1)|\geq| \sigma s \xi|\xi|^{\sigma-2}+y|-| x \mid+O(1),
$$

where $|x| \leq C R^{2}$ and $(y, \xi) \in \Gamma^{ \pm}\left(R^{3}, J_{3}, \tau_{3}\right)$. We then apply (5.33) with $\pm y \cdot \xi /|y \| \xi|>\tau_{3}$ and $\pm t= \pm \sigma s|\xi|^{\sigma-2} \geq 0$ to get

$$
\left.|\sigma s \xi| \xi\right|^{\sigma-2}+y \mid \geq C(|s|+|y|),
$$

for all $\pm s \geq 0$. We next use $|y|>R^{3}$ to control $|x| \lesssim R^{2}$ and obtain

$$
\left|\nabla_{\xi} \Phi^{ \pm}(R, s, x, y, \xi)\right| \geq C(1+|s|+|x|+|y|),
$$

for all $\pm s \geq 0$. By integrations by part with respect to $\xi$ with remark that higher derivatives of $\partial_{\xi} \Phi^{ \pm}$ are controlled by $\left|\nabla_{\xi} \Phi^{ \pm}\right|$, we get for all $N \geq 0$,

$$
\left|\chi\left(x / R^{2}\right) \int_{\mathbb{R}^{d}} e^{i h^{-1} \Phi^{ \pm}(R, s, x, y, \xi)} \breve{a}^{ \pm}(h, x, \xi) \overline{b^{ \pm}(h, y, \xi)} d \xi\right| \leq C h^{N}(1+|s|+|x|+|y|)^{-N} .
$$

By choosing $N$ large enough, we can dominate $\langle y\rangle^{m}$ and get

$$
\left|K_{h}^{ \pm}(s, x, y)\right| \leq C h^{N}(1+|s|+|x|+|y|)^{-N},
$$

for all $N$ large enough, therefore for all $N \geq 0$. We do the same for higher derivatives $\partial_{x}^{\alpha} \partial_{y}^{\beta} K_{h}(s, x, y)$ and the result follows. The kernel of the operator in the left hand side of (5.36) reads

$$
K_{h}^{ \pm}(s, x, y)=(2 \pi h)^{-d}\langle x\rangle^{m}(1-\chi)\left(x / R^{2}\right) \int_{\mathbb{R}^{d}} e^{i h^{-1} \Phi^{ \pm}(R, s, x, y, \xi)} \check{a}^{ \pm}(h, x, \xi) \overline{b^{ \pm}(h, y, \xi)} d \xi\langle y\rangle^{m} .
$$

We use the form of $\check{a}^{ \pm}(h)$ given in (5.23). In the case derivatives fall on $\kappa\left(x / R^{2}\right)$, we have that $|x| \leq C R^{2}$ and we can proceed as above. Note that we have from (5.33) with $\pm y \cdot \xi /|y \| \xi|>\sigma_{3}$ and $\pm t= \pm \sigma s|\xi|^{\sigma-2} \geq 0$ that

$$
\pm \frac{\left(y+\sigma s \xi|\xi|^{\sigma-2}\right) \xi}{\left.|y+\sigma s \xi| \xi\right|^{\sigma-2} \| \xi \mid}>\sigma_{3} \text { and }\left.|y+\sigma s \xi| \xi\right|^{\sigma-2} \mid \geq c_{ \pm}(|s|+|y|) .
$$

In the case derivatives fall on $\theta_{1 \rightarrow 2}$, we have

$$
\tau_{1}+\varepsilon \leq \pm \frac{x \cdot \xi}{|x \|| \xi \mid} \leq \tau_{2}-\varepsilon \text { or } \mp \frac{x \cdot \xi}{|x||\xi|} \geq-\tau_{2}+\varepsilon>-\tau_{2}+\varepsilon / 2
$$

By choosing $\varepsilon>0$ small enough such that $\tau_{3}-\tau_{2}+\varepsilon / 2>0$, (5.34) gives

$$
\left.|y+\sigma s \xi| \xi\right|^{\sigma-2}-x \mid \geq c\left(\left.|y+\sigma s \xi| \xi\right|^{\sigma-2}|+| x \mid\right) \geq C(|s|+|x|+|y|) .
$$


Thus $\left|\nabla_{\xi} \Phi^{ \pm}\right| \geq C(1+|s|+|x|+|y|)$ for $\pm s \geq 0$ and (5.36) follows as above.

2. The proof for the low frequency case is the same as above up to the conjugation by the unitary map $D_{\epsilon}$ in $L^{2}\left(\mathbb{R}^{d}\right)$. For instance, the kernel of the operator in the left hand side of (5.38) reads

$$
K_{\epsilon}^{ \pm}(s, x, y)=(2 \pi)^{-d} \chi\left(x / R^{2}\right) \int_{\mathbb{R}^{d}} e^{i \Phi_{\epsilon}^{ \pm}(R, s, x, y, \xi)} \check{a}^{ \pm}(\epsilon, x, \xi) \overline{b_{\epsilon}^{ \pm}(y, \xi)} d \xi\langle y\rangle^{m},
$$

where the phase $\Phi_{\epsilon}^{ \pm}(R, s, x, y, \xi)=S_{\epsilon, R}^{ \pm}(x, \xi)-\epsilon s|\xi|^{\sigma}-S_{\epsilon, R}^{ \pm}(y, \xi)$.

Lemma 5.13. Let $\sigma \in(0, \infty)$.

1. Under the notations of Theorem 5.8, for all $m \geq 0$ and all $N$ large enough, there exists $C>0$ such that for all $\pm s \geq 0$ and all $h \in(0,1]$,

$\left\|\langle x\rangle^{N / 8} J_{h}^{ \pm}\left(r_{N}^{ \pm}(h)\right) e^{-i s h^{-1}(h \Lambda)^{\sigma}} J_{h}^{ \pm}\left(b^{ \pm}(h)\right)^{\star}\langle x\rangle^{N / 4}\right\|_{\mathcal{L}\left(H^{-m}, H^{m}\right)} \leq C h^{-d-2 m}\langle s\rangle^{-N / 4}$.

2. Under the notations of Theorem 5.9, for all $N$ large enough, there exists $C>0$ such that for all $\pm s \geq 0$ and all $\epsilon \in(0,1]$,

$$
\left\|\langle\epsilon x\rangle^{N / 8} \mathcal{J}_{\epsilon}^{ \pm}\left(r_{N}^{ \pm}(\epsilon)\right) e^{-i \epsilon s \Lambda^{\sigma}} \mathcal{J}_{\epsilon}^{ \pm}\left(b_{\epsilon}^{ \pm}\right)^{\star}\langle\epsilon x\rangle^{N / 4}\right\|_{\mathcal{L}\left(L^{2}\right)} \leq C\langle\epsilon s\rangle^{-N / 4} .
$$

Proof. We only give the proof for the high frequency case, the low frequency one is similar. The kernel of the operator in the left hand side of (5.42) reads

$$
K_{h}^{ \pm}(s, x, y)=(2 \pi h)^{-d} \int_{\mathbb{R}^{d}} e^{i h^{-1} \Phi^{ \pm}(R, s, x, y, \xi)} A^{ \pm}(h, x, y, \xi) d \xi,
$$

where the amplitude $A^{ \pm}(h, x, y, \xi)=\langle x\rangle^{N / 8} r_{N}^{ \pm}(h, x, \xi) \overline{b^{ \pm}(h, y, \xi)}\langle y\rangle^{N / 4}$ and is compactly supported in $\xi$. We have from Proposition 5.1 and (5.41) that $\nabla_{\xi} \Phi^{ \pm}(R, s, x, y, \xi)=x-\sigma s \xi|\xi|^{\sigma-2}-y+O(1)$ and $\left.|\sigma s \xi| \xi\right|^{\sigma-2}+y \mid \geq C(|s|+|y|)$ for all $\pm s \geq 0$. By Peetre's inequality, we see that

$$
\left\langle\nabla_{\xi} \Phi^{ \pm}\right\rangle^{-1} \leq\langle x\rangle\left\langle y+\sigma s \xi|\xi|^{\sigma-2}\right\rangle^{-1} \leq C\langle x\rangle(\langle y\rangle+\langle s\rangle)^{-1} .
$$

We next write

$$
1=\chi\left(\nabla_{\xi} \Phi^{ \pm}\right)+(1-\chi)\left(\nabla_{\xi} \Phi^{ \pm}\right),
$$

where $\chi \in C_{0}^{\infty}\left(\mathbb{R}^{d}\right)$ with $\chi=1$ near 0 . Then $K_{h}^{ \pm}(s, x, y)$ is split into two terms. For the first term

$$
I_{1}=(2 \pi h)^{-d} \int_{\mathbb{R}^{d}} e^{i h^{-1} \Phi^{ \pm}(R, s, x, y, \xi)} \chi\left(\nabla_{\xi} \Phi^{ \pm}\right) A^{ \pm}(h, x, y, \xi) d \xi
$$

by using the fact that

$$
\begin{aligned}
\left|\chi\left(\nabla_{\xi} \Phi^{ \pm}\right)\right| & \leq C\left\langle\nabla_{\xi} \Phi^{ \pm}\right\rangle^{-3 N / 4} \leq C\langle x\rangle^{3 N / 4}(\langle y\rangle+\langle s\rangle)^{-3 N / 4} \\
& \leq C\langle x\rangle^{3 N / 4}\langle y\rangle^{-N / 2}\langle s\rangle^{-N / 4}
\end{aligned}
$$

and $A^{ \pm}(h, x, y, \xi)=O\left(\langle x\rangle^{-7 N / 8}\langle y\rangle^{N / 4}\right)$, it is bounded by $C h^{-d}\langle x\rangle^{-N / 8}\langle y\rangle^{-N / 4}\langle s\rangle^{-N / 4}$. For the second term

$$
I_{2}=(2 \pi h)^{-d} \int_{\mathbb{R}^{d}} e^{i h^{-1} \Phi^{ \pm}(R, s, x, y, \xi)}(1-\chi)\left(\nabla_{\xi} \Phi^{ \pm}\right) A^{ \pm}(h, x, y, \xi) d \xi
$$

thanks to the support of $(1-\chi)$, we can integrate by parts with respect to $\mathcal{L}:=\frac{h \nabla_{\xi} \Phi^{ \pm}}{i\left|\nabla_{\xi} \Phi^{ \pm}\right|^{2}} \circ \nabla_{\xi}$ to get many negative powers of $\left|\nabla_{\xi} \Phi^{ \pm}\right|$as we wish and estimate as in (5.44). Combine two terms and Schur's lemma, we have (5.42) for $m=0$. For $m \geq 1$, we can do the same with $\partial_{x}^{\alpha} \partial_{y}^{\beta} K_{h}^{ \pm}(s, x, y)$ with $|\alpha| \leq m,|\beta| \leq m$. This completes the proof.

Combining Lemma 5.12 and Lemma 5.13, we have the following result. 
Proposition 5.14. 1. Using the notations given in Theorem 5.8, for all $0 \leq m \leq d+1$ and all $N$ large enough, we can write for $k=2,3,4$,

$$
R_{k}^{ \pm}(N, t, h)=h^{N / 2} \int_{0}^{t} e^{-i(t-s) h^{-1} \psi\left(h^{2} P\right)}\langle x\rangle^{-N / 8} B_{m}^{ \pm}(N, s, h)\langle x\rangle^{-N / 4} d s,
$$

with

$$
\left\|B_{m}^{ \pm}(N, s, h)\right\|_{\mathcal{L}\left(H^{-m}, H^{m}\right)} \leq C\langle s\rangle^{-N / 4},
$$

for all $\pm s \geq 0$ and $h \in(0,1]$.

2. Using the notations given in Theorem 5.9 and for all $N$ large enough, we can write for $k=$ $2,3,4$,

$$
\mathcal{R}_{k}^{ \pm}(N, t, \epsilon)=\epsilon \int_{0}^{t} e^{-i(t-s) \epsilon \psi\left(\epsilon^{-2} P\right)}\langle\epsilon x\rangle^{-N / 8} \mathcal{B}_{N}^{ \pm}(s, \epsilon)\langle\epsilon x\rangle^{-N / 4} d s
$$

with

$$
\left\|\mathcal{B}_{N}^{ \pm}(s, \epsilon)\right\|_{\mathcal{L}\left(L^{2}\right)} \leq C\langle\epsilon s\rangle^{-N / 4}
$$

for all $\pm s \geq 0$ and all $\epsilon \in(0,1]$.

Proof. The cases $k=3,4$ follow immediately from Lemma 5.12 and Lemma 5.13. It remains to show the case $k=2$. Let us consider the high frequency case. We can write $R_{N}(h) E^{ \pm}(h)$ as

$$
\langle x\rangle^{-N / 8}\left(\langle x\rangle^{N / 8} R_{N}(h)\langle x\rangle^{7 N / 8}\right)\left(\langle x\rangle^{N / 8}\langle x\rangle^{-N} E^{ \pm}(h)\langle x\rangle^{N / 4}\right)\langle x\rangle^{-N / 4},
$$

where $E^{ \pm}(h):=J_{h}^{ \pm}\left(a^{ \pm}(h)\right) e^{-i s h^{-1}(h \Lambda)^{\sigma}} J_{h}^{ \pm}\left(b^{ \pm}(h)\right)^{\star}$. The first bracket is bounded in $\mathcal{L}\left(L^{2}\right)$ using Proposition 2.2. The second one is bounded in $\mathcal{L}\left(H^{-m}, H^{m}\right)$ using Lemma 5.13 with the fact that $\langle x\rangle^{-N} J_{h}^{ \pm}\left(a^{ \pm}(h)\right)=J_{h}^{ \pm}\left(\tilde{r}_{N}^{ \pm}(h)\right)$ where $\tilde{r}_{N}^{ \pm}(h)$ are bounded in $S(-N,-\infty)$. The low frequency case is similar using Proposition 2.7.

Next, we have the following micro-local propagation estimates both at high and low frequencies.

Proposition 5.15. Let $\sigma \in(0, \infty), f \in C_{0}^{\infty}(\mathbb{R} \backslash\{0\}), J_{4} \Subset(0,+\infty)$ be an open interval and $-1<$ $\tau_{4}<1$.

1. Consider $\mathbb{R}^{d}, d \geq 2$ equipped with a smooth metric $g$ satisfying (1.4),(1.5) and suppose that (1.12) is satisfied. Then for $R>0$ large enough and $\chi^{ \pm} \in S(0,-\infty)$ supported in $\Gamma^{ \pm}\left(R^{4}, J_{4}, \tau_{4}\right)$, we have the following estimates.

i. For all $m \in \mathbb{N}$ and all integer $l$ large enough, there exists $C>0$ such that for all $\pm t \leq 0$ and all $h \in(0,1]$,

$$
\left\|O p^{h}\left(\chi^{ \pm}\right)^{\star} e^{-i t h^{-1}\left(h \Lambda_{g}\right)^{\sigma}} f\left(h^{2} P\right)\langle x\rangle^{-l}\right\|_{\mathcal{L}\left(L^{2}, H^{m}\right)} \leq C h^{-m}\langle t\rangle^{-3 l / 4} .
$$

ii. For all $m \in \mathbb{N}$, all $\chi \in C_{0}^{\infty}\left(\mathbb{R}^{d}\right)$ and all $l \geq 1$, there exists $C>0$ such that for all $\pm t \leq 0$ and all $h \in(0,1]$,

$$
\left\|O p^{h}\left(\chi^{ \pm}\right)^{\star} e^{-i t h^{-1}\left(h \Lambda_{g}\right)^{\sigma}} f\left(h^{2} P\right) \chi\left(x / R^{2}\right)\right\|_{\mathcal{L}\left(L^{2}, H^{m}\right)} \leq C h^{l}\langle t\rangle^{-l} .
$$

iii. For all $\tilde{\chi}^{\mp} \in S(0,-\infty)$ supported in $\Gamma^{\mp}\left(R, J_{1}, \tilde{\tau}_{1}\right)$ with $-\tau_{4}<\tilde{\tau}_{1}<1$ and $J_{4} \Subset J_{1}$ and all $l \geq 1$, there exists $C>0$ such that for all $\pm t \leq 0$ and all $h \in(0,1]$,

$$
\left\|O p^{h}\left(\chi^{ \pm}\right)^{\star} e^{-i t h^{-1}\left(h \Lambda_{g}\right)^{\sigma}} f\left(h^{2} P\right) O p^{h}\left(\tilde{\chi}^{\mp}\right)\right\|_{\mathcal{L}\left(L^{\infty}\right)} \leq C h^{l}\langle t\rangle^{-l} .
$$

2. Consider $\mathbb{R}^{d}, d \geq 3$ equipped with a smooth metric g satisfying (1.4),(1.5). Let $\zeta \in C^{\infty}\left(\mathbb{R}^{d}\right)$ be supported outside $B(0,1)$ and equal to 1 near infinity. Then for $R>0$ large enough and all $\left(\chi_{\epsilon}^{ \pm}\right)_{\epsilon \in(0,1]}$ bounded families in $S(0,-\infty)$ supported in $\Gamma^{ \pm}\left(R^{4}, J_{4}, \tau_{4}\right)$, we have the following estimates. 
i. For all integer $l$ large enough, there exists $C>0$ such that for all $\pm t \leq 0$ and all $\epsilon \in(0,1]$,

$$
\left\|\zeta(\epsilon x) O p_{\epsilon}\left(\chi_{\epsilon}^{ \pm}\right)^{\star} e^{-i t \epsilon\left(\epsilon^{-1} \Lambda_{g}\right)^{\sigma}} f\left(\epsilon^{-2} P\right)\langle\epsilon x\rangle^{-l}\right\|_{\mathcal{L}\left(L^{2}\right)} \leq C\langle\epsilon t\rangle^{-3 l / 4}
$$

ii. For all $\chi \in C_{0}^{\infty}\left(\mathbb{R}^{d}\right)$ and all $l \geq 1$, there exists $C>0$ such that for all $\pm t \leq 0$ and all $\epsilon \in(0,1]$,

$$
\left\|\zeta(\epsilon x) O p_{\epsilon}\left(\chi_{\epsilon}^{ \pm}\right)^{\star} e^{-i t \epsilon\left(\epsilon^{-1} \Lambda_{g}\right)^{\sigma}} f\left(\epsilon^{-2} P\right) \chi\left(\epsilon x / R^{2}\right)\right\|_{\mathcal{L}\left(L^{2}\right)} \leq C\langle\epsilon t\rangle^{-l} .
$$

iii. For all $\tilde{\zeta} \in C^{\infty}\left(\mathbb{R}^{d}\right)$ supported outside $B(0,1)$ and equal to 1 near infinity and all $\left(\tilde{\chi}_{\epsilon}^{\mp}\right)_{\epsilon \in(0,1]}$ bounded families in $S(0,-\infty)$ supported in $\Gamma^{\mp}\left(R, J_{1}, \tilde{\tau}_{1}\right)$ with $-\tau_{4}<\tilde{\tau}_{1}<1$ and $J_{4} \Subset J_{1}$ and all $l \geq 1$, there exists $C>0$ such that for all $\pm t \leq 0$ and all $\epsilon \in(0,1]$,

$$
\left\|\zeta(\epsilon x) O p_{\epsilon}\left(\chi_{\epsilon}^{ \pm}\right)^{\star} e^{-i t \epsilon\left(\epsilon^{-1} \Lambda_{g}\right)^{\sigma}} f\left(\epsilon^{-2} P\right) O p_{\epsilon}\left(\tilde{\chi}_{\epsilon}^{\mp}\right) \tilde{\zeta}(\epsilon x)\right\|_{\mathcal{L}\left(L^{2}\right)} \leq C\langle\epsilon t\rangle^{-l} .
$$

Proof. We only give the proof for the low frequency case, the proof at high frequency is similar and essentially given in [8, Proposition 4.5].

i. We only consider the case $\chi_{\epsilon}^{+}$and $t \leq 0$, the case $\chi_{\epsilon}^{-}$and $t \geq 0$ is similar. By taking the adjoint, (5.50) is equivalent to

$$
\left\|\langle\epsilon x\rangle^{-l} f\left(\epsilon^{-2} P\right) e^{-i t \epsilon\left(\epsilon^{-1} \Lambda_{g}\right)^{\sigma}} O p_{\epsilon}\left(\chi_{\epsilon}^{+}\right) \zeta(\epsilon x)\right\|_{\mathcal{L}\left(L^{2}\left(\mathbb{R}^{d}\right)\right)} \leq C\langle\epsilon t\rangle^{-3 l / 4}, \quad t \geq 0,
$$

uniformly in $\epsilon \in(0,1]$. Thanks to the spectral localization, we can apply the Isozaki-Kitada parametrix given in Theorem 5.9 and obtain

$$
e^{-i t \epsilon\left(\epsilon^{-1} \Lambda_{g}\right)^{\sigma}} O p_{\epsilon}\left(\chi_{\epsilon}^{+}\right) \zeta(\epsilon x)=\mathcal{J}_{\epsilon}^{+}\left(a_{\epsilon}^{+}\right) e^{-i t \epsilon \Lambda^{\sigma}} \mathcal{J}_{\epsilon}^{+}\left(b_{\epsilon}^{+}\right)^{\star}+\mathcal{R}_{N}^{+}(t, \epsilon) .
$$

The main term can be written as

$$
\langle\epsilon x\rangle^{-l} f\left(\epsilon^{-2} P\right)\langle\epsilon x\rangle^{l}\langle\epsilon x\rangle^{-n}\langle\epsilon x\rangle^{n-l} \mathcal{J}_{\epsilon}^{+}\left(a_{\epsilon}^{+}\right) e^{-i t \epsilon \Lambda^{\sigma}} \mathcal{J}_{\epsilon}^{+}\left(b_{\epsilon}^{+}\right)^{\star}\langle\epsilon x\rangle^{n}\langle\epsilon x\rangle^{-n} .
$$

By using Corollary 2.9, we have the terms $\langle\epsilon x\rangle^{-l} f\left(\epsilon^{-2} P\right)\langle\epsilon x\rangle^{l}$ and $\langle\epsilon x\rangle^{-n}$ are bounded in $\mathcal{L}\left(L^{2}\right)$. It suffices to show for $l$ large enough,

$$
\left\|\langle\epsilon x\rangle^{n-l} \mathcal{J}_{\epsilon}^{+}\left(a_{\epsilon}^{+}\right) e^{-i t \epsilon \Lambda^{\sigma}} \mathcal{J}_{\epsilon}^{+}\left(b_{\epsilon}^{+}\right)^{\star}\langle\epsilon x\rangle^{n}\right\|_{\mathcal{L}\left(L^{2}\right)} \leq C\langle\epsilon t\rangle^{-3 l / 4}, \quad t \geq 0,
$$

uniformly in $\epsilon \in(0,1]$. This expected estimate follows by using the same process as in Lemma 5.13. We now study the remainders.

For $k=1$, we have

$$
\begin{aligned}
\left\|\langle\epsilon x\rangle^{-l} f\left(\epsilon^{-2} P\right) \mathcal{R}_{1}^{+}(N, t, \epsilon)\right\|_{\mathcal{L}\left(L^{2}\right)} & =\left\|\langle\epsilon x\rangle^{-l} f\left(\epsilon^{-2} P\right) e^{-i t \epsilon\left(\epsilon^{-1} \Lambda_{g}\right)^{\sigma}} O p_{\epsilon}\left(\tilde{r}_{N}^{+}(\epsilon)\right) \zeta(\epsilon x)\right\|_{\mathcal{L}\left(L^{2}\right)} \\
& \leq C\langle\epsilon t\rangle^{1-l} .
\end{aligned}
$$

Here we insert $\langle\epsilon x\rangle^{-l}\langle\epsilon x\rangle^{l}$ in the middle and use (2.23) and rescaled pseudo-differential calculus.

For $k=2,3,4$, Item 2 of Proposition 5.14 yields

$$
\langle\epsilon x\rangle^{-l} f\left(\epsilon^{-2} P\right) \mathcal{R}_{k}^{+}(N, t, \epsilon)=\epsilon \int_{0}^{t}\langle\epsilon x\rangle^{-l} f\left(\epsilon^{-2} P\right) e^{-i(t-s) \epsilon\left(\epsilon^{-1} \Lambda_{g}\right)^{\sigma}}\langle\epsilon x\rangle^{-N / 8} \mathcal{B}_{N}(s, \epsilon)\langle\epsilon x\rangle^{-N / 4} d s .
$$

Using again (2.23) and the fact that $\langle\epsilon x\rangle^{l-N / 8}$ and $\langle\epsilon x\rangle^{-N / 4}$ are of size $O_{\mathcal{L}\left(L^{2}\right)}(1)$ for $N$ large enough and (5.46), we obtain

$$
\left\|\langle\epsilon x\rangle^{-l} f\left(\epsilon^{-2} P\right) \mathcal{R}_{k}^{+}(N, t, \epsilon)\right\|_{\mathcal{L}\left(L^{2}\right)} \leq C \epsilon \int_{0}^{t}\langle\epsilon(t-s)\rangle^{1-l}\langle\epsilon s\rangle^{-N / 4} d s \leq C\langle\epsilon t\rangle^{1-l} .
$$

By choosing $l$ large enough such that $l-1 \geq 3 l / 4$, it shows (5.53).

ii. We do the same for (5.51), it is equivalent to show

$$
\left\|\chi\left(\epsilon x / R^{2}\right) f\left(\epsilon^{-2} P\right) e^{-i t \epsilon\left(\epsilon^{-1} \Lambda_{g}\right)^{\sigma}} O p_{\epsilon}\left(\chi_{\epsilon}^{+}\right) \zeta(\epsilon x)\right\|_{\mathcal{L}\left(L^{2}\left(\mathbb{R}^{d}\right)\right)} \leq C\langle\epsilon t\rangle^{-l}, \quad t \geq 0,
$$


uniformly in $\epsilon \in(0,1]$. We again use the Isozaki-Kitada parametrix. Let us firstly study remainder terms. We write the first remainder term $\chi\left(\epsilon x / R^{2}\right) f\left(\epsilon^{-2} P\right) \mathcal{R}_{1}^{+}(N, t, \epsilon)$ as

$$
\chi\left(\epsilon x / R^{2}\right)\langle\epsilon x\rangle^{l}\langle\epsilon x\rangle^{-l} f\left(\epsilon^{-2} P\right) e^{-i t \epsilon\left(\epsilon^{-1} \Lambda_{g}\right)^{\sigma}}\langle\epsilon x\rangle^{-l}\langle\epsilon x\rangle^{l} O p_{\epsilon}\left(\tilde{r}_{N}^{+}(\epsilon)\right) \zeta(\epsilon x) .
$$

Using (2.23) and the fact that $\chi\left(\epsilon x / R^{2}\right)\langle\epsilon x\rangle^{l}$ and $\langle\epsilon x\rangle^{l} O p_{\epsilon}\left(\tilde{r}_{N}^{+}(\epsilon)\right) \zeta(\epsilon x)$ are bounded in $\mathcal{L}\left(L^{2}\right)$ due to the support property of $\chi$ and rescaled pseudo-differential calculus given as in Proposition 2.7, we get

$$
\left\|\chi\left(\epsilon x / R^{2}\right) f\left(\epsilon^{-2} P\right) \mathcal{R}_{1}^{+}(N, t, \epsilon)\right\|_{\mathcal{L}\left(L^{2}\right)} \leq C\langle\epsilon t\rangle^{1-l} .
$$

For $k=2,3,4$, we have

$$
\left\|\chi\left(\epsilon x / R^{2}\right) f\left(\epsilon^{-2} P\right) \mathcal{R}_{k}^{+}(N, t, \epsilon)\right\|_{\mathcal{L}\left(L^{2}\right)} \leq C \epsilon \int_{0}^{t}\langle\epsilon(t-s)\rangle^{1-l}\langle\epsilon s\rangle^{-N / 4} d s \leq C\langle\epsilon t\rangle^{1-l} .
$$

For the main term, we can write

$$
\chi\left(\epsilon x / R^{2}\right)\langle\epsilon x\rangle^{l}\langle\epsilon x\rangle^{-l} f\left(\epsilon^{-2} P\right)\langle\epsilon x\rangle^{l}\langle\epsilon x\rangle^{-n}\langle\epsilon x\rangle^{n-l} \mathcal{J}_{\epsilon}^{+}\left(a_{\epsilon}^{+}\right) e^{-i t \epsilon \Lambda^{\sigma}} \mathcal{J}_{\epsilon}^{+}\left(b_{\epsilon}^{+}\right)^{\star}\langle\epsilon x\rangle^{n}\langle\epsilon x\rangle^{-n} .
$$

Thanks to the $L^{2}$-boundedness of $\chi\left(\epsilon x / R^{2}\right)\langle\epsilon x\rangle^{l},\langle\epsilon x\rangle^{-l} f\left(\epsilon^{-2} P\right)\langle\epsilon x\rangle^{l},\langle\epsilon x\rangle^{-n}$, it suffices to prove

$$
\left\|\langle\epsilon x\rangle^{n-l} \mathcal{J}_{\epsilon}^{+}\left(a_{\epsilon}^{+}\right) e^{-i t \epsilon \Lambda^{\sigma}} \mathcal{J}_{\epsilon}^{+}\left(b_{\epsilon}^{+}\right)^{\star}\langle\epsilon x\rangle^{n}\right\|_{\mathcal{L}\left(L^{2}\right)} \leq C\langle\epsilon t\rangle^{-l}, \quad t \geq 0,
$$

uniformly in $\epsilon \in(0,1]$. This expected estimate again follows from Lemma 5.12 by taking $l$ large enough. This proves (5.54).

iii. For (5.52), we firstly use the Isozaki-Kitada parametrix for $\tilde{\chi}_{\epsilon}^{-}$, namely

$$
e^{-i t \epsilon \psi\left(\epsilon^{-2} P\right)} O p_{\epsilon}\left(\tilde{\chi}_{\epsilon}^{-}\right) \tilde{\zeta}(\epsilon x)=\mathcal{J}_{\epsilon}^{-}\left(\tilde{a}_{\epsilon}^{-}\right) e^{-i t \epsilon \Lambda^{\sigma}} \mathcal{J}_{\epsilon}^{-}\left(\tilde{b}_{\epsilon}^{-}\right)^{\star}+\sum_{k=1}^{4} \tilde{\mathcal{R}}_{k}^{-}(N, t, \epsilon),
$$

where $\operatorname{supp}\left(\tilde{a}_{\epsilon}^{-}\right) \subset \Gamma^{-}\left(R^{1 / 4}, \tilde{J}_{1 / 4}, \tilde{\tau}_{1 / 4}\right)$ and $\operatorname{supp}\left(\tilde{b}_{\epsilon}^{-}\right) \subset \Gamma^{-}\left(R^{3 / 4}, \tilde{J}_{3 / 4}, \tilde{\tau}_{3 / 4}\right)$ with $\tilde{J}_{3 / 4} \Subset \tilde{J}_{1 / 4}$ small neighborhood of $J_{1}$ and $\tilde{\tau}_{1 / 4}, \tilde{\tau}_{3 / 4}$ can be chosen so that

$$
-1<-\tau_{4}<\tilde{\tau}_{1 / 4}<\tilde{\tau}_{3 / 4}<\tilde{\tau}_{1}<1
$$

Multiplying $\zeta(\epsilon x) O p_{\epsilon}\left(\chi_{\epsilon}^{+}\right)^{\star} f\left(\epsilon^{-2} P\right)$ to the left of $(5.55)$, the terms $\zeta(\epsilon x) O p_{\epsilon}\left(\chi_{\epsilon}^{+}\right)^{\star} f\left(\epsilon^{-2} P\right) \tilde{\mathcal{R}}_{k}^{-}(N, t, \epsilon)$ for $k=1,2,3,4$ satisfy the required estimate using the estimate (5.50), Lemma 5.12 and (5.46). Therefore, it remains to show

$$
\left\|\zeta(\epsilon x) O p_{\epsilon}\left(\chi_{\epsilon}^{+}\right)^{\star} f\left(\epsilon^{-2} P\right) \mathcal{J}_{\epsilon}^{-}\left(\tilde{a}_{\epsilon}^{-}\right) e^{-i t \epsilon \Lambda^{\sigma}} \mathcal{J}_{\epsilon}^{-}\left(\tilde{b}_{\epsilon}^{-}\right)^{\star}\right\|_{\mathcal{L}\left(L^{2}\right)} \leq C\langle\epsilon t\rangle^{-l}, \quad \pm t \leq 0,
$$

uniformly in $\epsilon \in(0,1]$. Thanks to the support of $\tilde{a}_{\epsilon}^{-}$, we can write $\mathcal{J}_{\epsilon}^{-}\left(\tilde{a}_{\epsilon}^{-}\right)=\zeta_{1}(\epsilon x) \mathcal{J}_{\epsilon}^{-}\left(\tilde{a}_{\epsilon}^{-}\right)$with $\zeta_{1} \in C^{\infty}\left(\mathbb{R}^{d}\right)$ supported outside $B(0,1)$ such that $\zeta_{1}(x)=1$ for $|x|>R^{1 / 4}$. The parametrix of $f\left(\epsilon^{-2} P\right) \zeta_{1}(\epsilon x)$ given in Proposition 2.7 and symbolic calculus give

$$
\zeta(\epsilon x) O p_{\epsilon}\left(\chi_{\epsilon}^{+}\right)^{\star} f\left(\epsilon^{-2} P\right) \zeta_{1}(\epsilon x)=O p_{\epsilon}\left(c_{\epsilon}^{+}\right)+B_{N}^{+}(\epsilon)\langle\epsilon x\rangle^{-N},
$$

where $\left(c_{\epsilon}^{+}\right)_{\epsilon \in(0,1]} \in S(0,-\infty)$ with $\operatorname{supp}\left(c_{\epsilon}^{+}\right) \subset \operatorname{supp}\left(\chi_{\epsilon}^{+}\right)$and $B_{N}^{+}(\epsilon)=O_{\mathcal{L}\left(L^{2}\right)}(1)$ uniformly in $\epsilon \epsilon$ $(0,1]$. We treat the remainder term by using Lemma 5.13 . For the main terms, we need to recall the following version of Proposition 5.4 which is essentially ${ }^{2}$ given in [8, Lemma 4.6].

Lemma 5.16. Given $J \Subset(0,+\infty),-1<\tau<1$ and the associated families of phase functions $\left(S_{\epsilon, R}^{ \pm}\right)_{R \gg 1}$ as in Proposition 5.1. Let $\left(a_{\epsilon}\right)_{\epsilon \in(0,1]}$ and $\left(c_{\epsilon}\right)_{\epsilon \in(0,1]}$ be bounded families in $S(0,-\infty)$. Then for all $N \geq 1$,

$$
O p_{\epsilon}\left(c_{\epsilon}\right) \mathcal{J}_{\epsilon}^{ \pm}\left(a_{\epsilon}\right)=\sum_{j=0}^{N-1} \mathcal{J}_{\epsilon}^{ \pm}\left(e_{\epsilon, j}\right)+\mathcal{J}_{\epsilon}^{ \pm}\left(e_{N}(\epsilon)\right)
$$

\footnotetext{
${ }^{2}$ See $(2.4),(5.3)$ and use Lemma 4.6 of [8] with $h=1$.
} 
where $\left(e_{\epsilon, j}\right)_{\epsilon \in(0,1]}$ and $\left(e_{N}(\epsilon)\right)_{\epsilon \in(0,1]}$ are bounded families in $S(0,-\infty)$ and $S(-N,-\infty)$ respectively. In particular, for all $\varepsilon>0$ small enough, by choosing $R>0$ large enough, we have

$$
\operatorname{supp}\left(c_{\epsilon}\right) \subset \Gamma^{ \pm}(R, J, \tau) \Longrightarrow \operatorname{supp}\left(e_{\epsilon, j}\right) \subset \Gamma^{ \pm}(R, J+(-\varepsilon, \varepsilon), \tau-\varepsilon)
$$

since $\nabla_{x} S_{\epsilon, R}^{ \pm}(x, \xi)=\xi+O\left(R^{-\rho}\right)$.

Using this lemma, we expand $O p_{\epsilon}\left(c_{\epsilon}^{+}\right) J_{\epsilon}^{-}\left(\tilde{a}_{\epsilon}^{-}\right)$and treat the remainder terms using again Lemma 5.13. It remains to prove the required estimate for the general term, namely

$$
\left\|\mathcal{J}_{\epsilon}^{-}\left(e_{\epsilon}^{+}\right) e^{-i t \epsilon \Lambda^{\sigma}} \mathcal{J}_{\epsilon}^{-}\left(\tilde{b}_{\epsilon}^{-}\right)^{\star}\right\|_{\mathcal{L}\left(L^{2}\right)} \leq C\langle\epsilon t\rangle^{-l}, \quad \pm t \leq 0
$$

uniformly in $\epsilon \in(0,1]$, where $\left(e_{\epsilon}^{+}\right)_{\epsilon \in(0,1]} \in S(0,-\infty)$ and $\operatorname{supp}\left(e_{\epsilon}^{+}\right) \in \Gamma^{+}\left(R^{4}, J_{4}+(-\varepsilon, \varepsilon), \tau_{4}-\varepsilon\right)$. Up to the conjugation by $D_{\epsilon}$, the kernel of the left hand side operator reads

$$
K_{\epsilon}(t, x, y)=(2 \pi)^{-d} \int_{\mathbb{R}^{d}} e^{i \Phi_{\epsilon}(R, t, x, y, \xi)} e_{\epsilon}^{+}(x, \xi) \overline{\tilde{b}_{\epsilon}^{-}(y, \xi)} d \xi
$$

where $\Phi_{\epsilon}(R, t, x, y, \xi)=S_{\epsilon, R}^{-}(x, \xi)-\epsilon t|\xi|^{\sigma}-S_{\epsilon, R}^{-}(y, \xi)$. Since $\operatorname{supp}\left(e_{\epsilon}^{+}\right) \subset \Gamma^{+}\left(R^{4}, J_{4}+(-\varepsilon, \varepsilon), \tau_{4}-\varepsilon\right)$ and $\operatorname{supp}\left(\tilde{b}_{\epsilon}^{-}\right) \subset \Gamma^{-}\left(R^{3 / 4}, \tilde{J}_{3 / 4}, \tilde{\tau}_{3 / 4}\right)$, we have

$$
\frac{x \cdot \xi}{|x \| \xi|}>\tau_{4}-\varepsilon, \quad-\frac{y \cdot \xi}{|y \| \xi|}>\tilde{\tau}_{3 / 4}
$$

By choosing $R>0$ large enough, we have that $\tau_{4}-\varepsilon+\tilde{\tau}_{3 / 4}>0$. Thus by Item 2 of Lemma 5.11 , we have

$$
\left|\nabla_{\xi} \Phi_{\epsilon}\right| \geq C(1+\epsilon|t|+|x|+|y|) .
$$

Using the non-stationary phase argument as in the proof of Lemma 5.12, we have

$$
\left\|\mathcal{J}_{\epsilon}^{+}\left(e_{\epsilon}^{+}\right) e^{-i t \epsilon \Lambda^{\sigma}} \mathcal{J}_{\epsilon}^{-}\left(\tilde{b}_{\epsilon}^{-}\right)^{\star}\right\|_{\mathcal{L}\left(L^{2}\right)} \leq C\langle\epsilon t\rangle^{-l}, \quad \pm t \leq 0
$$

uniformly in $\epsilon \in(0,1]$. The proof of Proposition 5.15 is now complete.

\subsection{Strichartz estimates.}

High frequencies. In this paragraph, we give the proof of (3.21). By scaling in time, it is in turn equivalent to prove

$$
\left\|(1-\chi) e^{-i t h^{-1}\left(h \Lambda_{g}\right)^{\sigma}} f\left(h^{2} P\right) u_{0}\right\|_{L^{p}\left(\mathbb{R}, L^{q}\right)} \leq C h^{-\kappa_{p, q}}\left\|f\left(h^{2} P\right) u_{0}\right\|_{L^{2}},
$$

where $\kappa_{p, q}=d / 2-d / q-1 / p$. By choosing $\tilde{f} \in C_{0}^{\infty}(\mathbb{R} \backslash 0)$ such that $\tilde{f}=1$ near $\operatorname{supp}(f)$, we can write for all $l \in \mathbb{N}$,

$$
(1-\chi) \tilde{f}\left(h^{2} P\right)=\sum_{k=0}^{N-1} h^{k} O p^{h}\left(a_{k}\right)^{\star}+h^{N} B_{N}(h)\langle x\rangle^{-l},
$$

where for $q \geq 2$,

$$
\left\|B_{N}(h)\right\|_{\mathcal{L}\left(L^{2}, L^{q}\right)} \leq C h^{-(d / 2-d / q)} .
$$

Thus $(1-\chi) e^{-i t h^{-1}\left(h \Lambda_{g}\right)^{\sigma}} f\left(h^{2} P\right) u_{0}$ becomes

$$
\sum_{k=0}^{N-1} h^{k} O p^{h}\left(a_{k}\right)^{\star} e^{-i t h^{-1}\left(h \Lambda_{g}\right)^{\sigma}} f\left(h^{2} P\right) u_{0}+h^{N} B_{N}(h)\langle x\rangle^{-l} e^{-i t h^{-1}\left(h \Lambda_{g}\right)^{\sigma}} f\left(h^{2} P\right) u_{0} .
$$

Using (5.56) and (2.20), $\left\|B_{N}(h)\langle x\rangle^{-l} e^{-i t h^{-1}\left(h \Lambda_{g}\right)^{\sigma}} f\left(h^{2} P\right) u_{0}\right\|_{L^{p}\left(\mathbb{R}, L^{q}\right)}$ is bounded by

$$
C h^{-(d / 2-d / q)}\left\|\langle x\rangle^{-l} e^{-i t h^{-1}\left(h \Lambda_{g}\right)^{\sigma}} f\left(h^{2} P\right) u_{0}\right\|_{L^{p}\left(\mathbb{R}, L^{2}\right)} \leq C h^{-(d / 2-d / q)+\left(1-N_{0}\right) / p}\left\|f\left(h^{2} P\right) u_{0}\right\|_{L^{2}} .
$$

Hence, by taking $N$ large enough, the remainder is bounded by $C h^{-\kappa_{p, q}}\left\|f\left(h^{2} P\right) u_{0}\right\|_{L^{2}}$. For the main terms, by choosing $\chi_{0} \in C_{0}^{\infty}\left(\mathbb{R}^{d}\right)$ such that $\chi_{0}=1$ for $|x| \leq 2$ and setting $\chi(x)=\chi_{0}\left(x / R^{4}\right)$, we see 
that $(1-\chi)$ is supported in $\left\{x \in \mathbb{R}^{d},|x| \geq 2 R^{4}>R^{4}\right\}$. For $R>0$ large enough and $\operatorname{supp}(\tilde{f})$ close enough to $\operatorname{supp}(f)$ and $J_{4} \Subset(0,+\infty)$ any open interval containing $\operatorname{supp}(f)$, we have

$$
\operatorname{supp}\left(a_{k}\right) \subset\left\{(x, \xi) \in \mathbb{R}^{2 d},|x|>R^{4},|\xi|^{2} \in J_{4}\right\}, \quad k=0, \ldots, N-1 .
$$

We want to show

$$
\left\|O p^{h}\left(a_{k}\right)^{\star} e^{-i t h^{-1}\left(h \Lambda_{g}\right)^{\sigma}} f\left(h^{2} P\right) u_{0}\right\|_{L^{p}\left(\mathbb{R}, L^{q}\right)} \leq C h^{-\kappa_{p, q}}\left\|f\left(h^{2} P\right) u_{0}\right\|_{L^{2}}, \quad k=0, \ldots, N-1 .
$$

Let us consider a general term, namely $O p^{h}(a)^{\star} e^{-i t h^{-1}\left(h \Lambda_{g}\right)^{\sigma}} f\left(h^{2} P\right) u_{0}$ with $a \in S(0,-\infty)$ satisfying (5.57). Next, by choosing a suitable partition of unity $\theta^{-}+\theta^{+}=1$ such that $\operatorname{supp}\left(\theta^{-}\right) \subset\left(-\infty,-\tau_{4}\right)$ and $\operatorname{supp}\left(\theta^{+}\right) \subset\left(\tau_{4},+\infty\right)$ and setting

$$
\chi^{ \pm}(x, \xi)=a(x, \xi) \theta^{ \pm}\left( \pm \frac{x \cdot \xi}{|x \| \xi|}\right)
$$

we have that $\chi^{ \pm} \in S(0,-\infty), \operatorname{supp}\left(\chi^{ \pm}\right) \subset \Gamma^{ \pm}\left(R^{4}, J_{4}, \tau_{4}\right)$ and

$$
O p^{h}(a)^{\star} e^{-i t h^{-1}\left(h \Lambda_{g}\right)^{\sigma}} f\left(h^{2} P\right) u_{0}=\left(O p^{h}\left(\chi^{-}\right)^{\star}+O p^{h}\left(\chi^{+}\right)^{\star}\right) e^{-i t h^{-1}\left(h \Lambda_{g}\right)^{\sigma}} f\left(h^{2} P\right) u_{0} .
$$

We only prove the estimate for $\chi^{+}$, i.e.

$$
\left\|O p^{h}\left(\chi^{+}\right)^{\star} e^{-i t h^{-1}\left(h \Lambda_{g}\right)^{\sigma}} f\left(h^{2} P\right) u_{0}\right\|_{L^{p}\left(\mathbb{R}, L^{q}\right)} \leq C h^{-\kappa_{p, q}}\left\|f\left(h^{2} P\right) u_{0}\right\|_{L^{2}},
$$

the one for $\chi^{-}$is similar. Since $O p^{h}\left(\chi^{+}\right)^{\star} e^{-i t h^{-1}\left(h \Lambda_{g}\right)^{\sigma}} f\left(h^{2} P\right)$ is bounded in $\mathcal{L}\left(L^{2}\right)$ uniformly in $h \in(0,1]$ and $t \in \mathbb{R}$, by Proposition 4.3, it suffices to prove the dispersive estimates, i.e.

$$
\left\|O p^{h}\left(\chi^{+}\right)^{\star} e^{-i t h^{-1}\left(h \Lambda_{g}\right)^{\sigma}} f^{2}\left(h^{2} P\right) O p^{h}\left(\chi^{+}\right)\right\|_{\mathcal{L}\left(L^{1}, L^{\infty}\right)} \leq C h^{-d}\left(1+|t| h^{-1}\right)^{-d / 2},
$$

for all $t \in \mathbb{R}$ uniformly in $h \in(0,1]$. By taking the adjoint, it reduces to prove

$$
\left\|O p^{h}\left(\chi^{+}\right)^{\star} e^{-i t h^{-1}\left(h \Lambda_{g}\right)^{\sigma}} f^{2}\left(h^{2} P\right) O p^{h}\left(\chi^{+}\right)\right\|_{\mathcal{L}\left(L^{1}, L^{\infty}\right)} \leq C h^{-d}\left(1+|t| h^{-1}\right)^{-d / 2},
$$

for all $t \leq 0$ uniformly in $h \in(0,1]$. We now prove (5.58). By using the Isozaki-Kitada parametrix with $J_{4}$ and $\tau_{4}$ as above together with arbitrary open intervals $J_{1}, J_{2}, J_{3}$ such that $J_{4} \Subset J_{3} \Subset J_{2} \Subset$ $J_{1} \Subset(0,+\infty)$ and arbitrary real numbers $\tau_{1}, \tau_{2}, \tau_{3}$ satisfying $-1<\tau_{1}<\tau_{2}<\tau_{3}<\tau_{4}<1$, the operator in the left hand side of (5.58) is written as

$$
O p^{h}\left(\chi^{+}\right)^{\star} f^{2}\left(h^{2} P\right)\left(J_{h}^{+}\left(a^{+}(h)\right) e^{-i t h^{-1}(h \Lambda)^{\sigma}} J_{h}^{+}\left(b^{+}(h)\right)^{\star}+\sum_{k=1}^{4} R_{k}^{+}(N, t, h)\right) .
$$

Using the fact that $O p^{h}\left(\chi^{+}\right)^{\star} f^{2}\left(h^{2} P\right)$ is bounded in $\mathcal{L}\left(L^{\infty}\right)$ and Proposition 5.10, we have

$$
\left\|O p^{h}\left(\chi^{+}\right)^{\star} f^{2}\left(h^{2} P\right) J_{h}^{+}\left(a^{+}(h)\right) e^{-i t h^{-1}(h \Lambda)^{\sigma}} J_{h}^{+}\left(b^{+}(h)\right)^{\star}\right\|_{\mathcal{L}\left(L^{1}, L^{\infty}\right)} \leq C h^{-d}\left(1+|t| h^{-1}\right)^{-d / 2},
$$

for all $t \in \mathbb{R}$ and $h \in(0,1]$. It remains to study the remainder terms.

For $k=1$, using the Sobolev embedding with $m>d / 2,(5.47)$ and the fact that $\langle x\rangle^{l} O p^{h}\left(\tilde{r}_{N}^{+}(h)\right)$ is of size $O_{\mathcal{L}\left(H^{-m}, L^{2}\right)}\left(h^{-m}\right)$ by pseudo-differential calculus, we have

$$
\left\|O p^{h}\left(\chi^{+}\right)^{\star} f^{2}\left(h^{2} P\right) R_{1}^{+}(N, t, h)\right\|_{\mathcal{L}\left(L^{1}, L^{\infty}\right)} \leq C h^{N-1-2 m}\langle t\rangle^{-3 l / 4} \leq C h^{-d}\left(1+|t| h^{-1}\right)^{-d / 2},
$$

for all $t \leq 0$ and all $h \in(0,1]$. The last estimate follows by taking $l=2 d / 3$ and $N$ large enough.

For $k=2$, by using (5.47) and the Sobolev embedding with $m>d / 2$, we have for $t-s \leq 0$,

$$
\left\|O p^{h}\left(\chi^{+}\right)^{\star} e^{-i(t-s) h^{-1}\left(h \Lambda_{g}\right)^{\sigma}} f^{2}\left(h^{2} P\right)\langle x\rangle^{-l}\right\|_{\mathcal{L}\left(L^{2}, L^{\infty}\right)} \leq C h^{-m}\langle t-s\rangle^{-3 l / 4} .
$$


We also have that $\langle x\rangle^{l} R_{N}(h)$ is bounded in $\mathcal{L}\left(L^{\infty}, L^{2}\right)$ due to Proposition 2.2 provided $N>l$. Thus for $N$ and $l$ large enough, Proposition 5.10 implies that

$$
\begin{aligned}
\| O p^{h}\left(\chi^{+}\right)^{\star} f^{2}\left(h^{2} P\right) & R_{2}^{+}(N, t, h) \|_{\mathcal{L}\left(L^{1}, L^{\infty}\right)} \\
\leq & C h^{N-1-m-d} \int_{0}^{t}\langle t-s\rangle^{-3 l / 4}\left(1+|s| h^{-1}\right)^{-d / 2} d s \leq C h^{-d}\left(1+|t| h^{-1}\right)^{-d / 2} .
\end{aligned}
$$

For $k=3$, by inserting $\langle x\rangle^{-l}\langle x\rangle^{l-N}\langle x\rangle^{N}$ and using the fact that $\langle x\rangle^{l-N}=O_{\mathcal{L}\left(L^{\infty}, L^{2}\right)}(1)$ for $N$ large enough, (5.59) and Proposition 5.10 with $J_{h}^{+}\left(a^{+}\right)=\langle x\rangle^{N} J_{h}^{+}\left(r_{N}^{+}(h)\right)$, we see that this remainder term satisfies the required estimate as for the second one.

For $k=4$, we rewrite $O p^{h}\left(\chi^{+}\right)^{\star} f^{2}\left(h^{2} P\right) R_{4}^{+}(N, t, h)$ as $-i h^{-1}$ times

$$
\int_{0}^{t} O p^{h}\left(\chi^{+}\right)^{\star} f^{2}\left(h^{2} P\right) e^{-i(t-s) h^{-1}\left(h \Lambda_{g}\right)^{\sigma}}(\chi+(1-\chi))\left(x / R^{2}\right) J_{h}^{+}\left(\check{a}^{+}(h)\right) e^{-i s h^{-1}(h \Lambda)^{\sigma}} J_{h}^{+}\left(b^{+}(h)\right)^{\star} d s,
$$

where $\chi \in C_{0}^{\infty}\left(\mathbb{R}^{d}\right)$ satisfying $\chi(x)=1$ for $|x| \leq 2$. The first term can be treated similarly as the second remainder using (5.48) instead of (5.47). For the second term, we need the following lemma (see [8, Proposition 5.2]).

Lemma 5.17. Choose $\tilde{\tau}_{1}$ such that $-\tau_{4}<\tilde{\tau}_{1}<-\tau_{2}$. If $R>0$ is large enough, we may choose $\tilde{\chi}^{-} \in S(0,-\infty)$ satisfying $\operatorname{supp}\left(\tilde{\chi}^{-}\right) \subset \Gamma^{-}\left(R, J_{1}, \tilde{\tau}_{1}\right)$ such that for all $m$ large enough,

$$
f\left(h^{2} P\right)(1-\chi)\left(x / R^{2}\right) J_{h}^{+}\left(\check{a}^{+}(h)\right)=O p^{h}\left(\tilde{\chi}^{-}\right) J_{h}^{+}\left(\tilde{e}_{m}(h)\right)+h^{m} \tilde{R}_{m}(h)
$$

where

$$
\tilde{R}_{m}(h)=J_{h}^{+}\left(\tilde{r}_{m}(h)\right)+\langle x\rangle^{-m / 2} R_{m}(h)\langle x\rangle^{-m / 2} J_{h}^{+}\left(\check{a}^{+}(h)\right),
$$

with $\left(\tilde{e}_{m}(h)\right)_{h \in(0,1]}$ and $\left(\tilde{r}_{m}(h)\right)_{h \in(0,1]}$ bounded families in $S(0,-\infty)$ and $S(-m,-\infty)$ respectively and $R_{m}(h)=O_{\mathcal{L}\left(L^{\infty}\right)}(1)$ uniformly in $h \in(0,1]$.

Using this lemma, the second term is written as $-i h^{-1}$ times

$$
\int_{0}^{t} O p^{h}\left(\chi^{+}\right)^{\star} e^{-i(t-s) h^{-1}\left(h \Lambda_{g}\right)^{\sigma}}\left(O p^{h}\left(\tilde{\chi}^{-}\right) J_{h}^{+}\left(\tilde{e}_{m}(h)\right)+h^{m} \tilde{R}_{m}(h)\right) e^{-i s h^{-1}(h \Lambda)^{\sigma}} J_{h}^{+}\left(b^{+}(h)\right)^{\star} d s .
$$

The remainder terms are treated similarly as the second remainder term using (5.47). The term involving $O p^{h}\left(\tilde{\chi}^{-}\right) J_{h}^{+}\left(\tilde{e}_{m}(h)\right)$ is studied by the same analysis as the second term using (5.49) instead of (5.47). This completes the proof.

Low frequencies. In this paragraph, we will prove (3.24). By scaling in time, it is equivalent to show

$$
\left\|(1-\chi)(\epsilon x) f\left(\epsilon^{-2} P\right) e^{-i t \epsilon\left(\epsilon^{-1} \Lambda_{g}\right)^{\sigma}} u_{0}\right\|_{L^{p}\left(\mathbb{R}, L^{q}\right)} \leq C \epsilon^{\kappa_{p, q}}\left\|f\left(\epsilon^{-2} P\right) u_{0}\right\|_{L^{2}},
$$

where $\kappa_{p, q}=d / 2-d / q-1 / p$. By choosing $\tilde{\tilde{f}} \in C_{0}^{\infty}(\mathbb{R} \backslash 0)$ such that $\tilde{\tilde{f}}=1$ near $\operatorname{supp}(f)$, we can write $(1-\chi)(\epsilon x) f\left(\epsilon^{-2} P\right)=(1-\chi)(\epsilon x) \tilde{\tilde{f}}\left(\epsilon^{-2} P\right) f\left(\epsilon^{-2} P\right)$. Next, we choose $\zeta \in C^{\infty}\left(\mathbb{R}^{d}\right)$ supported in $\mathbb{R}^{d} \backslash B(0,1)$ such that $\zeta=1$ near $\operatorname{supp}(1-\chi)$ and use Proposition 2.7 to have

$$
(1-\chi)(\epsilon x) \tilde{\tilde{f}}\left(\epsilon^{-2} P\right)=\sum_{k=0}^{N-1} \zeta(\epsilon x) O p_{\epsilon}\left(a_{\epsilon, k}\right)^{\star}+R_{N}(\epsilon),
$$

where $R_{N}(\epsilon)=\zeta(\epsilon x)\left(\epsilon^{-2} P+1\right)^{-N} B_{N}(\epsilon)\langle\epsilon x\rangle^{-N}$ with $\left(B_{N}(\epsilon)\right)_{\epsilon \in(0,1]}$ bounded $\mathcal{L}\left(L^{2}\right)$. Thus $(1-$ $\chi)(\epsilon x) f\left(\epsilon^{-2} P\right) e^{-i t \epsilon\left(\epsilon^{-1} \Lambda_{g}\right)^{\sigma}} u_{0}$ reads

$$
\sum_{k=0}^{N-1} \zeta(\epsilon x) O p_{\epsilon}\left(a_{\epsilon, k}\right)^{\star} e^{-i t \epsilon\left(\epsilon^{-1} \Lambda_{g}\right)^{\sigma}} f\left(\epsilon^{-2} P\right) u_{0}+R_{N}(\epsilon) e^{-i t \epsilon\left(\epsilon^{-1} \Lambda_{g}\right)^{\sigma}} f\left(\epsilon^{-2} P\right) u_{0} .
$$

We firstly consider the remainder term. 
Proposition 5.18. Let $N \geq(d-1) / 2+1$. Then for all $(p, q)$ fractional admissible, there exists $C>0$ such that for all $\epsilon \in(0,1]$,

$$
\left\|R_{N}(\epsilon) e^{-i t \epsilon\left(\epsilon^{-1} \Lambda_{g}\right)^{\sigma}} f\left(\epsilon^{-2} P\right) u_{0}\right\|_{L^{p}\left(\mathbb{R}, L^{q}\right)} \leq C \epsilon^{\kappa_{p, q}}\left\|u_{0}\right\|_{L^{2}}
$$

Proof. This result follows from the $T T^{\star}$ criterion given in Proposition 4.3 with $\epsilon^{-1}$ in place of $h$ and $T(t)=R_{N}(\epsilon) e^{-i t \epsilon\left(\epsilon^{-1} \Lambda_{g}\right)^{\sigma}} f\left(\epsilon^{-2} P\right)$. The $\mathcal{L}\left(L^{2}\right)$ bounds of $T(t)$ are obvious. Thus we need to prove the dispersive estimates. Using (2.6) with $q=\infty$ and (2.23) with $N \geq d / 2+1$, we have

$$
\begin{aligned}
\left\|T(t) T(s)^{\star}\right\|_{L^{1} \rightarrow L^{\infty}} & \leq C \epsilon^{d}\left\|\langle\epsilon x\rangle^{-N} e^{-i(t-s) \epsilon\left(\epsilon^{-1} \Lambda_{g}\right)^{\sigma}} f^{2}\left(\epsilon^{-2} P\right)\langle\epsilon x\rangle^{-N}\right\|_{\mathcal{L}\left(L^{2}\right)} \\
& \leq C \epsilon^{d}\langle\epsilon(t-s)\rangle^{1-N} \leq C \epsilon^{d}(1+\epsilon|t-s|)^{-d / 2} .
\end{aligned}
$$

This completes the proof.

For the main terms, by choosing $\chi_{0} \in C_{0}^{\infty}\left(\mathbb{R}^{d}\right)$ such that $\chi_{0}=1$ for $|x| \leq 2$ and setting $\chi(x)=$ $\chi_{0}\left(x / R^{4}\right)$, we see that $(1-\chi)$ is supported in $\left\{x \in \mathbb{R}^{d},|x|>R^{4}\right\}$. For $R>0$ large enough and $\operatorname{supp}(\tilde{f})$ close enough to $\operatorname{supp}(f)$ and $J_{4} \Subset(0,+\infty)$ any open interval containing $\operatorname{supp}(f)$, we have

$$
\operatorname{supp}\left(a_{\epsilon, k}\right) \subset\left\{(x, \xi) \in \mathbb{R}^{2 d},|x|>R^{4},|\xi|^{2} \in J_{4}\right\}, \quad k=0, \ldots, N-1 .
$$

We want to show for $k=0, \ldots, N-1$,

$$
\left\|\zeta(\epsilon x) O p_{\epsilon}\left(a_{\epsilon, k}\right)^{\star} e^{-i t \epsilon\left(\epsilon^{-1} \Lambda_{g}\right)^{\sigma}} f\left(\epsilon^{-2} P\right) u_{0}\right\|_{L^{p}\left(\mathbb{R}, L^{q}\right)} \leq C \epsilon^{\kappa_{p, q}}\left\|f\left(\epsilon^{-2} P\right) u_{0}\right\|_{L^{2}} .
$$

Let us consider the general term, namely $\zeta(\epsilon x) O p_{\epsilon}\left(a_{\epsilon}\right)^{\star} e^{-i t \epsilon\left(\epsilon^{-1} \Lambda_{g}\right)^{\sigma}} f\left(\epsilon^{-2} P\right) u_{0}$ with $\left(a_{\epsilon}\right)_{\epsilon \in(0,1]} \in$ $S(0,-\infty)$ satisfying (5.60). Next, by choosing a suitable partition of unity $\theta^{-}+\theta^{+}=1$ such that $\operatorname{supp}\left(\theta^{-}\right) \subset\left(-\infty,-\tau_{4}\right)$ and $\operatorname{supp}\left(\theta^{+}\right) \subset\left(\tau_{4},+\infty\right)$ and setting

$$
\chi_{\epsilon}^{ \pm}(x, \xi)=a_{\epsilon}(x, \xi) \theta^{ \pm}\left( \pm \frac{x \cdot \xi}{|x||\xi|}\right)
$$

we have that $\left(\chi_{\epsilon}^{ \pm}\right)_{\epsilon \in(0,1]} \in S(0,-\infty), \operatorname{supp}\left(\chi_{\epsilon}^{ \pm}\right) \subset \Gamma^{ \pm}\left(R^{4}, J_{4}, \tau_{4}\right)$ and

$$
\zeta(\epsilon x) O p_{\epsilon}\left(a_{\epsilon}\right)^{\star} e^{-i t \epsilon\left(\epsilon^{-1} \Lambda_{g}\right)^{\sigma}} f\left(\epsilon^{-2} P\right) u_{0}=\zeta(\epsilon x)\left(O p_{\epsilon}\left(\chi_{\epsilon}^{-}\right)^{\star}+O p_{\epsilon}\left(\chi_{\epsilon}^{+}\right)^{\star}\right) e^{-i t \epsilon\left(\epsilon^{-1} \Lambda_{g}\right)^{\sigma}} f\left(\epsilon^{-2} P\right) u_{0} .
$$

We only prove the estimate for $\chi_{\epsilon}^{+}$, i.e.

$$
\left\|\zeta(\epsilon x) O p_{\epsilon}\left(\chi_{\epsilon}^{+}\right)^{\star} e^{-i t \epsilon\left(\epsilon^{-1} \Lambda_{g}\right)^{\sigma}} f\left(\epsilon^{-2} P\right) u_{0}\right\|_{L^{p}\left(\mathbb{R}, L^{q}\right)} \leq C \epsilon^{\kappa_{p, q}}\left\|f\left(\epsilon^{-2} P\right) u_{0}\right\|_{L^{2}}
$$

the one for $\chi_{\epsilon}^{-}$is similar. By $T T^{\star}$ criterion and that $T(t):=\zeta(\epsilon x) O p_{\epsilon}\left(\chi_{\epsilon}^{+}\right)^{\star} e^{-i t \epsilon\left(\epsilon^{-1} \Lambda_{g}\right)^{\sigma}} f\left(\epsilon^{-2} P\right)$ is bounded in $\mathcal{L}\left(L^{2}\right)$ for all $t \in \mathbb{R}$ and all $\epsilon \in(0,1]$, it suffices to prove dispersive estimates, i.e.

$$
\left\|\zeta(\epsilon x) O p_{\epsilon}\left(\chi_{\epsilon}^{+}\right)^{\star} e^{-i t \epsilon\left(\epsilon^{-1} \Lambda_{g}\right)^{\sigma}} f^{2}\left(\epsilon^{-2} P\right) O p_{\epsilon}\left(\chi_{\epsilon}^{+}\right) \zeta(\epsilon x)\right\|_{\mathcal{L}\left(L^{1}, L^{\infty}\right)} \leq C \epsilon^{d}(1+\epsilon|t|)^{-d / 2},
$$

for all $t \in \mathbb{R}$ uniformly in $\epsilon \in(0,1]$. By taking the adjoint, it reduces to prove

$$
\left\|\zeta(\epsilon x) O p_{\epsilon}\left(\chi_{\epsilon}^{+}\right)^{\star} e^{-i t \epsilon\left(\epsilon^{-1} \Lambda_{g}\right)^{\sigma}} f^{2}\left(\epsilon^{-2} P\right) O p_{\epsilon}\left(\chi_{\epsilon}^{+}\right) \zeta(\epsilon x)\right\|_{\mathcal{L}\left(L^{1}, L^{\infty}\right)} \leq C \epsilon^{d}(1+\epsilon|t|)^{-d / 2},
$$

for all $t \leq 0$ uniformly in $\epsilon \in(0,1]$. Let us prove (5.61). For simplicity, we set

$$
A_{\epsilon}^{+}:=\zeta(\epsilon x) O p_{\epsilon}\left(\chi_{\epsilon}^{+}\right)^{\star} f^{2}\left(\epsilon^{-2} P\right) .
$$

Using the Isozaki-Kitada parametrix given in Theorem 5.9, we see that

$$
A_{\epsilon}^{+} e^{-i t \epsilon\left(\epsilon^{-1} \Lambda_{g}\right)^{\sigma}} O p_{\epsilon}\left(\chi_{\epsilon}^{+}\right) \zeta(\epsilon x)=A_{\epsilon}^{+}\left(\mathcal{J}_{\epsilon}^{+}\left(a_{\epsilon}^{+}\right) e^{-i t \epsilon \Lambda^{\sigma}} \mathcal{J}_{\epsilon}^{+}\left(b_{\epsilon}^{+}\right)^{\star}+\sum_{k=1}^{4} \mathcal{R}_{k}^{+}(N, t, \epsilon)\right) .
$$

We firstly note that $A_{\epsilon}^{+}$is bounded in $\mathcal{L}\left(L^{\infty}\right)$. Indeed, we write

$$
\zeta(\epsilon x) O p_{\epsilon}\left(\chi_{\epsilon}^{+}\right)^{\star} f^{2}\left(\epsilon^{-2} P\right)=\zeta(\epsilon x) O p_{\epsilon}\left(\chi_{\epsilon}^{+}\right)^{\star} \zeta_{1}(\epsilon x) f^{2}\left(\epsilon^{-2} P\right),
$$


V. D. DINH

where $\zeta_{1} \in C^{\infty}\left(\mathbb{R}^{d}\right)$ is supported outside $B(0,1)$ satisfying $\zeta_{1}(x)=1$ for $|x|>R^{4}$. This is possible since $O p_{\epsilon}\left(\chi_{\epsilon}^{+}\right)=\zeta_{1}(\epsilon x) O p_{\epsilon}\left(\chi_{\epsilon}^{+}\right)$. The factors $\zeta(\epsilon x) O p_{\epsilon}\left(\chi_{\epsilon}^{+}\right)^{\star}$ and $\zeta_{1}(\epsilon x) f^{2}\left(\epsilon^{-2} P\right)$ are bounded in $\mathcal{L}\left(L^{\infty}\right)$ by the rescaled pseudo-differential operator and Corollary 2.8 respectively. Thanks to the $\mathcal{L}\left(L^{\infty}\right)$-bound of $A_{\epsilon}^{+}$and (5.29), we have dispersive estimates for the main terms. It remains to prove dispersive estimates for remainder terms. By rescaled pseudo-differential calculus, we can write for $l>d / 2$,

$$
A_{\epsilon}^{+}=\tilde{\zeta}(\epsilon x)\left(\epsilon^{-2} P+1\right)^{-l}\left(\zeta(\epsilon x) O p_{\epsilon}\left(\tilde{\chi}_{\epsilon}^{+}\right)^{\star}+\tilde{B}_{l}^{+}(\epsilon)\langle\epsilon x\rangle^{-l}\right) f^{2}\left(\epsilon^{-2} P\right),
$$

where $\tilde{\zeta} \in C^{\infty}\left(\mathbb{R}^{d}\right)$ is supported outside $B(0,1)$ and equal to 1 near $\operatorname{supp}(\zeta)$ and $\left(\tilde{\chi}_{\epsilon}^{+}\right)_{\epsilon \in(0,1]} \in S(0,-\infty)$ satisfying $\operatorname{supp}\left(\tilde{\chi}_{\epsilon}^{+}\right) \subset \operatorname{supp}\left(\chi_{\epsilon}^{+}\right)$and $\tilde{B}_{l}^{+}(\epsilon)=O_{\mathcal{L}\left(L^{2}\right)}(1)$ uniformly in $\epsilon \in(0,1]$. This follows by expanding $\left(\epsilon^{-2} P+1\right)^{l} \zeta(\epsilon x) O p_{\epsilon}\left(\chi_{\epsilon}^{+}\right)^{\star}$ by rescaled pseudo-differential calculus.

For $k=1$, using the Proposition 2.7, we can write

$$
\mathcal{R}_{1}^{+}(N, t, \epsilon)=e^{-i t \epsilon\left(\epsilon^{-1} \Lambda_{g}\right)^{\sigma}}\langle\epsilon x\rangle^{-N} B_{N}^{+}(\epsilon)\left(\epsilon^{-2} P+1\right)^{-N} \zeta(\epsilon x),
$$

where $B_{N}^{+}(\epsilon)=O_{\mathcal{L}\left(L^{2}\right)}(1)$ uniformly in $\epsilon \in(0,1]$. Then, using Proposition 2.6 with $q=\infty$ and (5.50), we have

$$
\begin{aligned}
\left\|\tilde{\zeta}(\epsilon x)\left(\epsilon^{-2} P+1\right)^{-l} \zeta(\epsilon x) O p_{\epsilon}\left(\tilde{\chi}_{\epsilon}^{+}\right)^{\star} f^{2}\left(\epsilon^{-2} P\right) \mathcal{R}_{1}^{+}(N, t, \epsilon)\right\|_{\mathcal{L}\left(L^{1}, L^{\infty}\right)} & \leq C \epsilon^{d}\langle\epsilon t\rangle^{-3 N / 4} \\
& \leq C \epsilon^{d}(1+\epsilon|t|)^{-d / 2}
\end{aligned}
$$

for all $t \leq 0$ and all $\epsilon \in(0,1]$ provided $N$ is taken large enough. Moreover, using again Proposition 2.6 and (2.23), we also have

$$
\begin{aligned}
\left\|\tilde{\zeta}(\epsilon x)\left(\epsilon^{-2} P+1\right)^{-l} \tilde{B}_{l}(\epsilon)\langle\epsilon x\rangle^{-l} f^{2}\left(\epsilon^{-2} P\right) \mathcal{R}_{1}^{+}(N, t, \epsilon)\right\|_{\mathcal{L}\left(L^{1}, L^{\infty}\right)} & \leq C \epsilon^{d}\langle\epsilon t\rangle^{1-l} \\
& \leq C \epsilon^{d}(1+\epsilon|t|)^{-d / 2},
\end{aligned}
$$

for all $t \leq 0$ and all $\epsilon \in(0,1]$ provided $l$ and $N$ are taken large enough. This implies

$$
\left\|A_{\epsilon}^{+} \mathcal{R}_{1}^{+}(N, t, \epsilon)\right\|_{\mathcal{L}\left(L^{1}, L^{\infty}\right)} \leq C \epsilon^{d}(1+\epsilon|t|)^{-d / 2},
$$

for all $t \leq 0$ and all $\epsilon \in(0,1]$.

Next, thanks to the support of $b_{\epsilon}^{+}$, we can write

$$
\mathcal{J}_{\epsilon}^{+}\left(b_{\epsilon}^{+}\right)^{\star}=\mathcal{J}_{\epsilon}^{+}\left(\tilde{b}_{\epsilon}^{+}\right)^{\star}\left(\epsilon^{-2} P+1\right)^{-N} \zeta_{1}(\epsilon x),
$$

where $\left(\tilde{b}_{\epsilon}^{+}\right)_{\epsilon \in(0,1]} \in S(0,-\infty), \operatorname{supp}\left(\tilde{b}_{\epsilon}^{+}\right) \subset \Gamma^{+}\left(R^{3}, J_{3}, \sigma_{3}\right)$ and $\zeta_{1} \in C^{\infty}\left(\mathbb{R}^{d}\right)$ is supported outside $B(0,1)$ such that $\zeta_{1}(x)=1$ for $|x|>R^{3}$. Indeed, we write for $\tilde{\zeta}_{1} \in C^{\infty}\left(\mathbb{R}^{d}\right)$ supported outside $B(0,1)$ and $\tilde{\zeta}_{1}=1$ in $\operatorname{supp}\left(\zeta_{1}\right)$,

$$
\mathcal{J}_{\epsilon}^{+}\left(b_{\epsilon}^{+}\right)^{\star}=\mathcal{J}_{\epsilon}^{+}\left(b_{\epsilon}^{+}\right)^{\star} \tilde{\zeta}_{1}(\epsilon x)\left(\epsilon^{-2} P+1\right)^{N}\left(\left(\epsilon^{-2} P+1\right)^{-N} \zeta_{1}(\epsilon x)\right) .
$$

We have (5.62) by taking the adjoint of $\left(\epsilon^{-2} P+1\right)^{N} \tilde{\zeta}_{1}(\epsilon x) \mathcal{J}_{\epsilon}^{+}\left(b_{\epsilon}^{+}\right)=\mathcal{J}_{\epsilon}^{+}\left(\tilde{b}_{\epsilon}^{+}\right)$.

For $k=2$, using (2.6) and its adjoint, (5.50), (5.62), $\langle\epsilon x\rangle^{l} R_{N}(\epsilon)\langle\epsilon x\rangle^{N-l}=O_{\mathcal{L}\left(L^{2}\right)}(1)$ and estimating as in Lemma 5.13, we have

$$
\begin{aligned}
\| \tilde{\zeta}(\epsilon x)\left(\epsilon^{-2} P+1\right)^{-l} \zeta(\epsilon x) O p_{\epsilon}\left(\tilde{\chi}_{\epsilon}^{+}\right)^{\star} & f^{2}\left(\epsilon^{-2} P\right) \mathcal{R}_{2}^{+}(N, t, \epsilon) \|_{\mathcal{L}\left(L^{1}, L^{\infty}\right)} \\
& \leq C \epsilon^{d} \epsilon \int_{0}^{t}\langle\epsilon(t-s)\rangle^{-3 l / 4}\langle\epsilon s\rangle^{-N / 4} d s \leq C \epsilon^{d}(1+\epsilon|t|)^{-d / 2}
\end{aligned}
$$


for $t \leq 0$ provided that $l$ and $N$ are taken large enough. Moreover, using (2.23) instead of (5.50), we have

$$
\begin{aligned}
\| \tilde{\zeta}(\epsilon x)\left(\epsilon^{-2} P+1\right)^{-l} \tilde{B}_{l}(\epsilon)\langle\epsilon x\rangle^{-l} & f^{2}\left(\epsilon^{-2} P\right) \mathcal{R}_{2}^{+}(N, t, \epsilon) \|_{\mathcal{L}\left(L^{1}, L^{\infty}\right)} \\
& \leq C \epsilon^{d} \epsilon \int_{0}^{t}\langle\epsilon(t-s)\rangle^{1-l}\langle\epsilon s\rangle^{-N / 4} d s \leq C \epsilon^{d}(1+\epsilon|t|)^{-d / 2},
\end{aligned}
$$

for all $t \leq 0$ and all $\epsilon \in(0,1]$. This implies

$$
\left\|A_{\epsilon}^{+} \mathcal{R}_{2}^{+}(N, t, \epsilon)\right\|_{\mathcal{L}\left(L^{1}, L^{\infty}\right)} \leq C \epsilon^{d}(1+\epsilon|t|)^{-d / 2}, \quad \forall t \leq 0, \epsilon \in(0,1]
$$

The third remainder term is treated similarly as the second one. It remains to study the last remainder term. To do so, we split

$$
A_{\epsilon}^{+} \mathcal{R}_{4}^{+}(N, t, \epsilon)=-i \int_{0}^{t} A_{\epsilon}^{+} e^{-i(t-s) \epsilon\left(\epsilon^{-1} \Lambda_{g}\right)^{\sigma}}(\chi+(1-\chi))\left(\epsilon x / R^{2}\right) \mathcal{J}_{\epsilon}^{+}\left(\check{a}^{+}(\epsilon)\right) e^{-i s \epsilon \Lambda^{\sigma}} \mathcal{J}_{\epsilon}^{+}\left(b_{\epsilon}^{+}\right)^{\star} d s,
$$

where $\chi \in C_{0}^{\infty}\left(\mathbb{R}^{d}\right)$ satisfying $\chi(x)=1$ for $|x| \leq 2$. The first term can be treated similarly as the second remainder using (5.51) instead of (5.50) and Lemma 5.12. For the second term, we need the following lemma (see [8, Proposition 5.2]).

Lemma 5.19. Choose $\tilde{\tau}_{1}$ such that $-\tau_{4}<\tilde{\tau}_{1}<-\tau_{2}$. If $R>0$ is large enough, we may choose a bounded family of symbols $\tilde{\chi}_{\epsilon}^{-} \in S(0,-\infty)$ satisfying $\operatorname{supp}\left(\tilde{\chi}_{\epsilon}^{-}\right) \subset \Gamma^{-}\left(R, J_{1}, \tilde{\tau}_{1}\right)$ and $\tilde{\zeta}_{2} \in C^{\infty}\left(\mathbb{R}^{d}\right)$ supported outside $B(0,1)$ satisfying $\tilde{\zeta}_{2}=1$ on $\operatorname{supp}(1-\chi)$ such that for all $m$ large enough,

$$
f\left(\epsilon^{-2} P\right)(1-\chi)\left(\epsilon x / R^{2}\right) \mathcal{J}_{\epsilon}^{+}\left(\check{a}^{+}(\epsilon)\right)=O p_{\epsilon}\left(\tilde{\chi}_{\epsilon}^{-}\right) \tilde{\zeta}_{2}(\epsilon x) \mathcal{J}_{\epsilon}^{+}\left(\tilde{e}_{m}(\epsilon)\right)+\tilde{R}_{m}(\epsilon),
$$

where

$$
\tilde{R}_{m}(\epsilon)=\mathcal{J}_{\epsilon}^{+}\left(\tilde{r}_{m}(\epsilon)\right)+\langle\epsilon x\rangle^{-m / 2} R_{m}(\epsilon)\langle\epsilon x\rangle^{-m / 2} \mathcal{J}_{\epsilon}^{+}\left(\check{a}^{+}(\epsilon)\right),
$$

with $\left(\tilde{e}_{m}(\epsilon)\right)_{\epsilon \in(0,1]}$ and $\left(\tilde{r}_{m}(\epsilon)\right)_{\epsilon \in(0,1]}$ bounded families in $S(0,-\infty)$ and $S(-m,-\infty)$ respectively and $R_{m}(\epsilon)=O_{\mathcal{L}\left(L^{2}\right)}(1)$ uniformly in $\epsilon \in(0,1]$.

We set

$$
A_{\epsilon}^{+}=\left(A_{\epsilon, 1}^{+}+A_{\epsilon, 2}^{+}\right) f\left(\epsilon^{-2} P\right)
$$

where

$$
\begin{aligned}
& A_{\epsilon, 1}^{+}=\tilde{\zeta}(\epsilon x)\left(\epsilon^{-2} P+1\right)^{-l} \zeta(\epsilon x) O p_{\epsilon}\left(\tilde{\chi}_{\epsilon}^{+}\right)^{\star} f\left(\epsilon^{-2} P\right), \\
& A_{\epsilon, 2}^{+}=\tilde{\zeta}(\epsilon x)\left(\epsilon^{-2} P+1\right)^{-l} \tilde{B}_{l}(\epsilon)\langle\epsilon x\rangle^{-l} f\left(\epsilon^{-2} P\right) .
\end{aligned}
$$

Using Lemma 5.19, we firstly consider

$$
-i h^{-1} \int_{0}^{t} A_{\epsilon, 1}^{+} e^{-i(t-s) \epsilon\left(\epsilon^{-1} \Lambda_{g}\right)^{\sigma}}\left(O p_{\epsilon}\left(\tilde{\chi}_{\epsilon}^{-}\right) \tilde{\zeta}_{2}(\epsilon x) \mathcal{J}_{\epsilon}^{+}\left(\tilde{e}_{m}(\epsilon)\right)+\tilde{R}_{m}(\epsilon)\right) e^{-i s \epsilon \Lambda^{\sigma}} \mathcal{J}_{\epsilon}^{+}\left(b_{\epsilon}^{+}\right)^{\star} d s .
$$

The remainder terms are treated similarly as the second remainder term using (5.50) and Lemma 5.13. The term involving $O p_{\epsilon}\left(\tilde{\chi}_{\epsilon}^{-}\right) \tilde{\zeta}_{2}(\epsilon x) \mathcal{J}_{\epsilon}^{+}\left(\tilde{e}_{m}(\epsilon)\right)$ is studied by the same analysis as the second term using (5.52) instead of (5.47). For the term

$$
-i h^{-1} \int_{0}^{t} A_{\epsilon, 2}^{+} e^{-i(t-s) \epsilon\left(\epsilon^{-1} \Lambda_{g}\right)^{\sigma}}\left(O p_{\epsilon}\left(\tilde{\chi}_{\epsilon}^{-}\right) \tilde{\zeta}_{2}(\epsilon x) \mathcal{J}_{\epsilon}^{+}\left(\tilde{e}_{m}(\epsilon)\right)+\tilde{R}_{m}(\epsilon)\right) e^{-i s \epsilon \Lambda^{\sigma}} \mathcal{J}_{\epsilon}^{+}\left(b_{\epsilon}^{+}\right)^{\star} d s,
$$

the required estimate follows by using (2.23) and Lemma 5.13. This completes the proof. 


\section{Inhomogeneous Strichartz estimates}

In this section, we will give the proofs of Proposition 1.5 and Proposition 1.7. The main tool is the homogeneous Strichartz estimates (1.15) and the so called Christ-Kiselev Lemma. To do so, we recall the following result (see [13] or [42]).

Lemma 6.1. Let $X$ and $Y$ be Banach spaces and assume that $K(t, s)$ is a continuous function taking its values in the bounded operators from $Y$ to $X$. Suppose that $-\infty \leq c<d \leq \infty$, and set

$$
A f(t)=\int_{c}^{d} K(t, s) f(s) d s .
$$

Assume that

Define the operator $\tilde{A}$ as

$$
\|A f\|_{L^{q}([c, d], X)} \leq C\|f\|_{L^{p}([c, d], Y)} .
$$

$$
\tilde{A} f(t)=\int_{c}^{t} K(t, s) f(s) d s,
$$

Then for $1 \leq p<q \leq \infty$, there exists $\tilde{C}>0$ such that

$$
\|\tilde{A} f\|_{L^{q}([c, d], X)} \leq \tilde{C}\|f\|_{L^{p}([c, d], Y)} .
$$

We are now able to prove the inhomogeneous Strichartz estimates (1.17) and (1.21).

Inhomogeneous Strichartz estimates for fractional Schrödinger equation. We give the proof of Proposition 1.5 by following a standard argument (see e.g. [50]). Let $u$ be the solution to (1.1). By Duhamel formula, we have

$$
u(t)=e^{-i t \Lambda_{g}^{\sigma}} u_{0}-i \int_{0}^{t} e^{-i(t-s) \Lambda_{g}^{\sigma}} F(s) d s=: u_{\mathrm{hom}}(t)+u_{\mathrm{inh}}(t) .
$$

Using (1.15), we have

$$
\left\|u_{\mathrm{hom}}\right\|_{L^{p}\left(\mathbb{R}, L^{q}\right)} \leq C\left\|u_{0}\right\|_{\dot{H}_{g}^{\gamma_{p}, q}} .
$$

It remains to prove the inhomogeneous part, namely

$$
\left\|\int_{0}^{t} e^{-i(t-s) \Lambda_{g}^{\sigma}} F(s) d s\right\|_{L^{p}\left(\mathbb{R}, L^{q}\right)} \leq C\|F\|_{L^{a^{\prime}}\left(\mathbb{R}, L^{b^{\prime}}\right)},
$$

where $(p, q),(a, b)$ are fractional admissible pairs satisfying $(p, a) \neq(2,2)$ and the gap condition (1.18). By the Christ-Kiselev Lemma, it suffices to prove

$$
\left\|\int_{\mathbb{R}} e^{-i(t-s) \Lambda_{g}^{\sigma}} F(s) d s\right\|_{L^{p}\left(\mathbb{R}, L^{q}\right)} \leq C\|F\|_{L^{a^{\prime}}\left(\mathbb{R}, L^{b^{\prime}}\right)},
$$

for all fractional admissible pairs satisfying (1.18) excluding the case $p=a^{\prime}=2$. We now prove (6.1). Define

$$
T_{\gamma_{p, q}}: u_{0} \in \mathscr{L}_{g} \mapsto \Lambda_{g}^{-\gamma_{p, q}} e^{-i t \Lambda_{g}^{\sigma}} u_{0} \in L^{p}\left(\mathbb{R}, L^{q}\right) .
$$

Thanks to (1.15), we see that $T_{\gamma_{p, q}}$ is a bounded operator. Similar result holds for $T_{\gamma_{a, b}}$. Next, we take the adjoint for $T_{\gamma_{a, b}}$ and obtain a bounded operator

$$
T_{\gamma_{a, b}}^{\star}: F \in L^{a^{\prime}}\left(\mathbb{R}, L^{b^{\prime}}\right) \mapsto \int_{\mathbb{R}} \Lambda_{g}^{-\gamma_{a, b}} e^{i s \Lambda_{g}^{\sigma}} F(s) d s \in \mathscr{L}_{g}^{\prime},
$$

where $\mathscr{L}_{g}^{\prime}$ is the dual space of $\mathscr{L}_{g}$. Using $(1.18)$ or $\gamma_{a, b}=-\gamma_{a^{\prime}, b^{\prime}}-\sigma=-\gamma_{p, q}$, we have

$$
\left\|\int_{\mathbb{R}} e^{-i(t-s) \Lambda_{g}^{\sigma} F(s) d s}\right\|_{L^{p}\left(\mathbb{R}, L^{q}\right)}=\left\|T_{\gamma_{p, q}} T_{\gamma_{a, b}}^{\star} F\right\|_{L^{p}\left(\mathbb{R}, L^{q}\right)} \leq C\|F\|_{L^{a^{\prime}}\left(\mathbb{R}, L^{b^{\prime}}\right)},
$$


and (6.1) follows.

Next, we prove

$$
\|u\|_{L^{\infty}\left(\mathbb{R}, \dot{H}_{g}^{\gamma_{p, q}}\right)} \leq C\left(\left\|u_{0}\right\|_{\dot{H}_{g}^{\gamma_{p}, q}}+\|F\|_{L^{a^{\prime}}\left(\mathbb{R}, L^{b^{\prime}}\right)}\right) .
$$

By using the homogeneous Strichartz estimate for a fractional admissible pair $(\infty, 2)$ with $\gamma_{\infty, 2}=0$ and that $\|u\|_{L^{\infty}\left(\mathbb{R}, \dot{H}_{g}^{\left.\gamma_{p, q}\right)}\right.}=\left\|\Lambda_{g}^{\gamma_{p, q}} u\right\|_{L^{\infty}\left(\mathbb{R}, L^{2}\right)}$, we have

$$
\|u\|_{L^{\infty}\left(\mathbb{R}, \dot{H}_{g}^{\left.\gamma_{p, q}\right)}\right.} \leq C\left(\left\|\Lambda_{g}^{\gamma_{p, q}} u_{0}\right\|_{L^{2}}+\left\|\int_{0}^{t} \Lambda_{g}^{\gamma_{p, q}} e^{-i(t-s) \Lambda_{g}^{\sigma}} F(s) d s\right\|_{L^{\infty}\left(\mathbb{R}, L^{2}\right)}\right) .
$$

Using the Christ-Kiselev Lemma, it suffices to prove

$$
\left\|\int_{\mathbb{R}} \Lambda_{g}^{\gamma_{p, q}} e^{-i(t-s) \Lambda_{g}^{\sigma} F(s) d s}\right\|_{L^{\infty}\left(\mathbb{R}, L^{2}\right)} \leq C\|F\|_{L^{a^{\prime}}\left(\mathbb{R}, L^{b^{\prime}}\right)} .
$$

Using the above notation, we have

$$
\begin{aligned}
\| \int_{\mathbb{R}} \Lambda_{g}^{\gamma_{p, q}} e^{-i(t-s) \Lambda_{g}^{\sigma} F(s) d s \|_{L^{\infty}\left(\mathbb{R}, L^{2}\right)}} & =\left\|T_{0} T_{\gamma_{a, b}}^{\star} F\right\|_{L^{\infty}\left(\mathbb{R}, L^{2}\right)} \\
& \leq C\left\|T_{\gamma_{a, b}}^{\star} F\right\|_{L^{2}} \leq C\|F\|_{L^{a^{\prime}}\left(\mathbb{R}, L^{b^{\prime}}\right)} .
\end{aligned}
$$

This completes the proof of Proposition 1.5.

Inhomogeneous Strichartz estimates for fractional wave equation. We give the proof of Proposition 1.7. Let $v$ be the solution to (1.20). By Duhamel formula, we have

$$
v(t)=\cos t \Lambda_{g}^{\sigma} u_{0}+\frac{\sin t \Lambda_{g}^{\sigma}}{\Lambda_{g}^{\sigma}} u_{1}+\int_{0}^{t} \frac{\sin (t-s) \Lambda_{g}^{\sigma}}{\Lambda_{g}^{\sigma}} F(s) d s=: v_{\text {hom }}(t)+v_{\text {inh }}(t),
$$

where $v_{\text {hom }}$ is the sum of first two terms and $v_{\text {inh }}$ is the last one. We firstly prove

$$
\|v\|_{L^{p}\left(\mathbb{R}, L^{q}\right)} \leq C\left(\left\|v_{0}\right\|_{\dot{H}_{g}^{\gamma p, q}}+\left\|v_{1}\right\|_{\dot{H}_{g}^{\gamma_{p}, q}-\sigma}+\|F\|_{L^{a^{\prime}}\left(\mathbb{R}, L^{b^{\prime}}\right)}\right) .
$$

By observing that

$$
\cos t \Lambda_{g}^{\sigma}=\frac{e^{i t \Lambda_{g}^{\sigma}}+e^{-i t \Lambda_{g}^{\sigma}}}{2}, \quad \sin t \Lambda_{g}^{\sigma}=\frac{e^{i t \Lambda_{g}^{\sigma}}-e^{-i t \Lambda_{g}^{\sigma}}}{2 i}
$$

and using (1.15), we have

$$
\left\|v_{\mathrm{hom}}\right\|_{L^{p}\left(\mathbb{R}, L^{q}\right)} \leq C\left(\left\|v_{0}\right\|_{\dot{H}_{g}^{\gamma_{p}, q}}+\left\|v_{1}\right\|_{\dot{H}_{g}^{\gamma_{p}, q}-\sigma}\right) .
$$

Let us prove the inhomogeneous part which is in turn equivalent to

$$
\left\|\int_{0}^{t} \frac{e^{-i(t-s) \Lambda_{g}^{\sigma}}}{\Lambda_{g}^{\sigma}} F(s) d s\right\|_{L^{p}\left(\mathbb{R}, L^{q}\right)} \leq C\|F\|_{L^{a^{\prime}}\left(\mathbb{R}, L^{b^{\prime}}\right)},
$$

where $(p, q),(a, b)$ are fractional admissible satisfying the gap condition (1.22). We define the operator

$$
T_{\gamma_{p, q}}: u_{0} \in \mathscr{L}_{g} \mapsto \Lambda_{g}^{-\gamma_{p, q}} e^{-i t \Lambda_{g}^{\sigma}} u_{0} \in L^{p}\left(\mathbb{R}, L^{q}\right) .
$$

Thanks to (1.15), we see that $T_{\gamma_{p, q}}$ is a bounded operator. Next, we take the adjoint for $T_{\gamma_{a, b}}$ and obtain a bounded operator

$$
T_{\gamma_{a, b}}^{\star}: F \in L^{a^{\prime}}\left(\mathbb{R}, L^{b^{\prime}}\right) \mapsto \int_{\mathbb{R}} \Lambda_{g}^{-\gamma_{a, b}} e^{i s \Lambda_{g}^{\sigma}} F(s) d s \in \mathscr{L}_{g}^{\prime} .
$$

Using (1.22) or $\gamma_{a, b}=-\gamma_{a^{\prime}, b^{\prime}}-\sigma=-\gamma_{p, q}+\sigma$, we have

$$
\left\|\int_{\mathbb{R}} \frac{e^{-i(t-s) \Lambda_{g}^{\sigma}}}{\Lambda_{g}^{\sigma}} F(s) d s\right\|_{L^{p}\left(\mathbb{R}, L^{q}\right)}=\left\|T_{\gamma_{p, q}} T_{\gamma_{a, b}}^{\star} F\right\|_{L^{p}\left(\mathbb{R}, L^{q}\right)} \leq C\|F\|_{L^{a^{\prime}}\left(\mathbb{R}, L^{b^{\prime}}\right)} .
$$


As in the proof of the inhomogeneous Strichartz estimates for the fractional Schrödinger equations, the Christ-Kiselev Lemma implies (6.2) for all fractional admissible pairs satisfying the gap condition (1.22) excluding the case $p=a^{\prime}=2$.

Next, we prove

$$
\|v\|_{L^{\infty}\left(\mathbb{R}, \dot{H}_{g}^{\gamma_{p}, q}\right)} \leq C\left(\left\|v_{0}\right\|_{\dot{H}_{g}^{\gamma_{p}, q}}+\left\|v_{1}\right\|_{\dot{H}_{g}^{\gamma_{p, q}-\sigma}}+\|F\|_{L^{a^{\prime}}\left(\mathbb{R}, L^{b^{\prime}}\right)}\right) .
$$

By using the homogeneous Strichartz estimate for a fractional admissible pair $(\infty, 2)$ with $\gamma_{\infty, 2}=0$ and that $\|v\|_{L^{\infty}\left(\mathbb{R}, \dot{H}_{g}^{\gamma_{p}, q}\right)}=\left\|\Lambda_{g}^{\gamma_{p, q}} v\right\|_{L^{\infty}\left(\mathbb{R}, L^{2}\right)}$, we have

$$
\begin{aligned}
\|v\|_{L^{\infty}\left(\mathbb{R}, \dot{H}_{g}^{\gamma_{p, q}}\right)} \leq C\left(\left\|\Lambda_{g}^{\gamma_{p, q}} v_{0}\right\|_{L^{2}}+\left\|\Lambda_{g}^{\gamma_{p, q}} v_{1}\right\|_{\dot{H}_{g}^{-\sigma}}\right. & \\
& \left.+\left\|\int_{0}^{t} \Lambda_{g}^{\left(\gamma_{p, q}-\sigma\right)} \sin (t-s) \Lambda_{g}^{\sigma} F(s) d s\right\|_{L^{\infty}\left(\mathbb{R}, L^{2}\right)}\right) .
\end{aligned}
$$

Using the Christ-Kiselev Lemma, it suffices to prove

$$
\left\|\int_{\mathbb{R}} \Lambda_{g}^{\left(\gamma_{p, q}-\sigma\right)} e^{-i(t-s) \Lambda_{g}^{\sigma} F(s) d s}\right\|_{L^{\infty}\left(\mathbb{R}, L^{2}\right)} \leq C\|F\|_{L^{a^{\prime}}\left(\mathbb{R}, L^{b^{\prime}}\right)} .
$$

Using the above notation, we have

$$
\begin{aligned}
\| \int_{\mathbb{R}} \Lambda_{g}^{\left(\gamma_{p, q}-\sigma\right)} e^{-i(t-s) \Lambda_{g}^{\sigma} F(s) d s \|_{L^{\infty}\left(\mathbb{R}, L^{2}\right)}} & =\left\|T_{0} T_{\gamma_{a, b}}^{\star} F\right\|_{L^{\infty}\left(\mathbb{R}, L^{2}\right)} \\
& \leq C\left\|T_{\gamma_{a, b}}^{\star} F\right\|_{L^{2}} \leq C\|F\|_{L^{a^{\prime}}\left(\mathbb{R}, L^{b^{\prime}}\right)} .
\end{aligned}
$$

We repeat the same process for $\partial_{t} v$ and obtain

$$
\left\|\partial_{t} v\right\|_{L^{\infty}\left(\mathbb{R}, \dot{H}_{g}^{\gamma_{p}, q-\sigma}\right)} \leq C\left(\left\|v_{0}\right\|_{\dot{H}_{g}^{\gamma_{p}, q}}+\left\|v_{1}\right\|_{\dot{H}_{g}^{\gamma_{p, q}-\sigma}}+\|F\|_{L^{\alpha^{\prime}}\left(\mathbb{R}, L^{b^{\prime}}\right)}\right) .
$$

This completes the proof of Proposition 1.7.

\section{ACKNOWLEDGMENTS}

The author would like to express his deep thanks to his wife - Uyen Cong for her encouragement and support. He would like to thank his supervisor Prof. Jean-Marc Bouclet for the kind guidance, encouragement and careful reading of the manuscript. He also would like to thank the reviewer for his/her helpful comments and suggestions.

\section{REFERENCES}

[1] J. Bergh, J. Löfstöm, Interpolation spaces, Springer, New York (1976). 3

[2] J. M. Bouclet, Distributions spectrales pour des opérateurs perturbés, PhD Thesis, Nantes University (2000). 26, 27,28

[3] J. M. Bouclet, Spectral distributions for long range perturbations, J. Funct. Anal. 212, 431-471 (2004). 27, 28

[4] J. M. Bouclet, Low frequency estimates and local energy decay for asymptotically euclidean Laplacians, Comm. in PDE. 36, No. 7, 1239-1286 (2011). 4

[5] J. M. Bouclet, H. Mizutani, Global in time Strichartz inequalities on asymptotically conic manifolds with temperate trapping, arXiv:1602.06287 (2016). 2, 4, 5, 7, 8, 11, 13, 14, 17

[6] J. M. Bouclet, J. Royer, Sharp low frequency estimates on asymptotically conical manifolds, Comm. Math. Phys. 335, No. 2, 809-850 (2015). 5, 11

[7] J. M. Bouclet, N. Tzvetkov, Strichartz estimates for long range perturbations, Amer. J. Math 129, 1565-1609 (2007). 5, 7, 8, 25

[8] J. M. Bouclet, N. Tzvetkov, On global Strichartz estimates for non-trapping metrics, J. Funct. Anal. 254, 1661-1682 (2008). 2, 5, 13, 34, 35, 38, 39, 42, 45

[9] J. M. Bouclet, Strichartz estimates on asymptotically hyperbolic manifolds, Analysis \& PDE 4, No. 1, 1-84 (2011). 5 
[10] N. Burq, Semi-classical estimates for the resolvent in non-trapping geometries, International Math. Res. Notices, 221-241 (2002). 4

[11] N. Burq, C. Guillarmou, A. Hassell, Strichartz estimates without loss on manifolds with hyperbolic trapped geodesics, Geom. Funct. Anal. vol. 20, 627-656 (2010). 4

[12] I. Chavel, Eigenvalues in Riemannian geometry, Pure and Applied Mathematics 115, Academic Press Inc., Orlando, FL (1984). 14

[13] M. Christ, A. Kiselev, Maximal functions associated to filtrations, J. Funct. Anal. 179, 409-425 (2001). 46

[14] K. Datchev, Local smoothing for scattering manifolds with hyperbolic trapped sets, Comm. Math. Phys. vol. 286, No. 3, 837-850 (2009). 4

[15] M. Dimassi, J. Sjöstrand, Spectral asymptotics in the semi-classical limit, London Math. Soc. Lecture Note, vol.268, Cambridge University Press (1999). 9

[16] V. D. Dinh, Well-posedness of nonlinear fractional Schrödinger and wave equations in Sobolev spaces, arXiv:1609.06181 (2016). 1, 2, 6

[17] V. D. Dinh, Strichartz estimates for the fractional Schrödinger and wave equations on compact manifolds without boundary, J. Differential Equations 263, No. 12, 8804-8837 (2017). 2, 4, 20, 21

[18] J. Ginibre, G. Velo, The global Cauchy problem for the nonlinear Klein-Gordon equation, Math. Z. 189, 487-505 (1985). 3

[19] A. Grigor'yan, Estimates of heat kernel on Riemannian manifolds, in: "Spectral theory and geometry. ICMS Instructional Conference, Edinburgh 1998", Ed. E. B. Davies and Yu. Safarov, London Math. Soc. Lecture Note Ser. 273, Cambridge Univ. Press, 140-225 (1999). 14, 15

[20] G. H. Hardy, J. E. Littlewood, Some properties of fractional integrals, Math. Z. 27, 565-606 (1928). 16

[21] A. Hassell, J. Zhang, Global in time Strichartz estimates on non-trapping asymptotically conic manifolds, Anal. PDE 9, No. 1, 151-192 (2016). 2

[22] A. D. Ionescu, F. Pusateri, Nonlinear fractional Schrödinger equations in one dimension, J. Func. Anal. 266, 139-176 (2014). 1

[23] H. Isozaki, H. Kitada, Modified wave operators with time independent modifiers, J. Fac. Sci. Univ. Tokyo. 32 77- 104 (1985). 5

[24] V.I. Karpman, Stabilization of soliton instabilities by higher-order dispersion: Fourth order nonlinear Schrödinger-type equations, Phys. Rev. E 53 (2), 1336-1339 (1996). 1

[25] V. I. Karpman, A. G. Shagalov, Stability of soliton described by nonlinear Schrödinger-type equations with higher-order dispersion, Phys. D 144, 194-210 (2000). 1

[26] M. Keel, T. Tao, Endpoint Strichartz estimates, Amer. J. Math. 120, No. 5, 955-980 (1998). 21

[27] N. Laskin, Fractional quantum mechanis and Lévy path integrals, Phys. Lett A 268, 298-305 (2000). 1

[28] N. Laskin, Fractional Schrödinger equation, Phys. Rev. E 66, 056108 (2002). 1

[29] J. Marzuola, J. Metcalfe, D. Tataru, Strichartz estimates and local smoothing estimates for asymptotically flat Schrödinger equations, J. Funct. Anal. 255, No. 6, 1497-1553 (2008). 2

[30] H. Mizutani, Strichartz estimates for Schrödinger equations with variable coefficients and potentials at most linear at spatial infinity, J. Math. Soc. Japan. 65, No.3, 687-721 (2013). 5

[31] H. Mizutani, Strichartz estimates for Schrödinger equations on scattering manifolds, Comm. Partial Differential Equations 37, 169-224 (2012). 5

[32] Q. H. Nguyen, Sharp Strichartz estimates for water waves systems, to appear in Trans. Amer. Math. Soc, 2018. 1

[33] S. Nonnenmacher, M. Zworski, Quantum decay rates in chaotic scattering, Acta Math. vol. 203, No. 2, 149-224 (2009). 4

[34] M. Reed, B. Simon, Methods of modern mathematical physics, Volume IV: Analysis of operators , Academic Press (1978). 12

[35] D. Robert, Autour de l'approximation semi-classique, Progress in mathematics 68, Birkhaüser (1987). 8,26

[36] D. Robert, Asymptotique de la phase de diffusion à haute énergie pour les perturbations du second ordre du Laplacien, An. Scient. Éc. Norm. Sup. 25, 107-134 (1992). 4

[37] D. Robert, Relative time delay for perturbations of elliptic operators and semi-classical asymptotics, J. Funct. Anal 126, 36-82 (1994). 11, 25

[38] M. Ruzhansky, M. Sugimoto, Weighted Sobolev L $L^{2}$ estimates for a class of Fourier integral operators, Math. Nachr. 284, 1715-1738 (2011). 26

[39] L. Saloff-Coste, Aspects of Sobolev-type inequalities, London Math. Soc. Lecture Ser. 289, Cambridge University Press, Cambridge (2002). 15

[40] S. L. Sobolev, On a theorem of functional analysis, Mat. Sb. 4, 471-479 (1938) (English version: AMS Transl. Ser. 2, No. 34, 39-68 (1963)). 16

[41] C. D. Sogge, Fourier integrals in classical analysis, Cambridge tracts in Mathematics (1993). 3 
[42] C. D. Sogge, Lectures on nonlinear wave equations, Second edition, International Press (2008). 46

[43] C. D. Sogge, C. Wang, Concerning the wave equation on asymptotically Euclidean manifolds, J. Anal. 112, No. 1, 1-32 (2010). 4

[44] G. Staffilani, D. Tataru, Strichartz estimates for a Schrödinger operator with non-smooth coefficients, Comm. Partial Differential Equations 27, 1337-1372 (2002). 4

[45] D. Tataru, Parametrices and dispersive estimates for Schrödinger operators with variable coefficients, Amer. J. Math. 130, No. 3, 571-634 (2008). 2

[46] D. Tataru, H. Koch, Carleman estimates and absence of embedded eigenvalues, Comm. Math. Phys. 267, No. 2, 419-449 (2006). 14

[47] H. Triebel, Theory of function spaces, Basel: Birkhäuser (1983). 3

[48] G. Vodev, Local energy decay of solutions to the wave equation for non-trapping metrics, Ark. Mat. 42, 379-397 (2004). 4

[49] M. Zworski, Semiclassical Analysis, Graduate Studies in Mathematics 138, AMS (2012). 21

[50] J. Zhang, Strichartz estimates and nonlinear wave equation on non-trapping asymptotically conic manifolds, Advances in Mathematics 271, 91-111 (2015). 21, 46

[51] J. Zhang, J. Zheng, Global-in-time Strichartz estimates for Schrödinger on scattering manifolds, Comm. Partial Differential Equations 42 (2017), No. 12, 1962-1981. 2

[52] J. Zhang, J. Zheng, Global-in-time Strichartz estimates and cubic Schrödinger equation on metric cone, Preprint arXiv:1702.05813, 2018. 2

(V. D. Dinh) Institut de Mathématiques de Toulouse, Université Toulouse III Paul Sabatier, 31062 Toulouse Cedex 9, France and Department of Mathematics, HCMC University of Pedagogy, 280 An Duong Vuong, Ho Chi Minh, Vietnam

E-mail address: dinhvan.duong@math.univ-toulouse.fr 